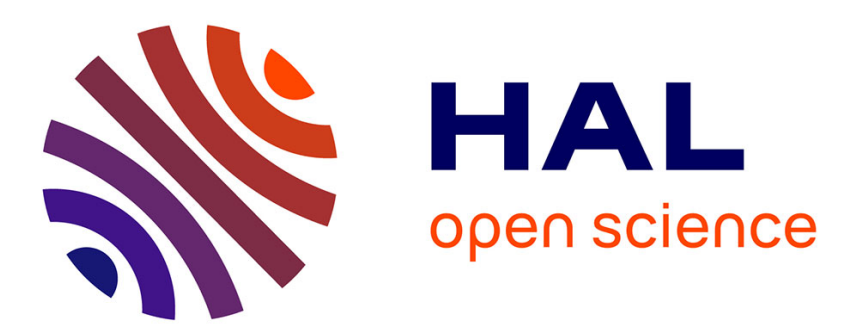

\title{
Efficient numerical schemes for viscoplastic avalanches. Part 1: the 1D case
}

\author{
Enrique D. Fernandez-Nieto, J.M. Gallardo, Paul Vigneaux
}

\section{To cite this version:}

Enrique D. Fernandez-Nieto, J.M. Gallardo, Paul Vigneaux. Efficient numerical schemes for viscoplastic avalanches. Part 1: the 1D case. Journal of Computational Physics, 2014, 264, pp.55-90. 10.1016/j.jcp.2014.01.026 . hal-01011373

\section{HAL Id: hal-01011373 \\ https://hal.science/hal-01011373}

Submitted on 9 Oct 2017

HAL is a multi-disciplinary open access archive for the deposit and dissemination of scientific research documents, whether they are published or not. The documents may come from teaching and research institutions in France or abroad, or from public or private research centers.
L'archive ouverte pluridisciplinaire HAL, est destinée au dépôt et à la diffusion de documents scientifiques de niveau recherche, publiés ou non, émanant des établissements d'enseignement et de recherche français ou étrangers, des laboratoires publics ou privés. 


\title{
Efficient numerical schemes for viscoplastic avalanches. Part 1: the 1D case ch $^{\frac{\pi}{\sqrt{3}}}$
}

\author{
Enrique D. Fernández-Nieto ${ }^{\mathrm{a}, *}$, José M. Gallardo $^{\mathrm{b}}$, Paul Vigneaux ${ }^{\mathrm{c}}$ \\ ${ }^{a}$ Departamento de Matemática Aplicada I, Universidad de Sevilla. E.T.S. Arquitectura. Avda, Reina Mercedes, s/n. 41012 Sevilla, Spain \\ ${ }^{b}$ Departamento de Análisis Matemático, Universidad de Málaga. F. Matemáticas, Campus Teatinos S/N, Spain \\ ${ }^{c}$ Unitée de Mathématiques Pures et Appliquées, Ecole Normale Supérieure de Lyon. 46 allée d'Italie. 69364 Lyon Cedex 07. France
}

\begin{abstract}
This paper deals with the numerical resolution of a shallow water viscoplastic flow model. Viscoplastic materials are characterized by the existence of a yield stress: below a certain critical threshold in the imposed stress, there is no deformation and the material behaves like a rigid solid, but when that yield value is exceeded, the material flows like a fluid. In the context of avalanches, it means that after going down a slope, the material can stop and its free surface has a non trivial shape, as opposed to the case of water (Newtonian fluid). The model involves variational inequalities associated to the yield threshold: finite-volume schemes are used together with duality methods (namely Augmented Lagrangian and Bermúdez-Moreno) to discretize the problem. To be able to accurately simulate the stopping behaviour of the avalanche, new schemes need to be designed, involving the classical notion of wellbalancing. In the present context, it needs to be extended to take into account the viscoplastic nature of the material as well as general bottoms with wet/dry fronts which are encountered in geophysical geometries. We derived such schemes and numerical experiments are presented to show their performances.
\end{abstract}

Keywords: Viscoplastic, Shallow Water, Finite Volume, Well-Balanced, Variational inequality, Bingham

\section{Introduction}

The objective of this paper is to present improved numerical schemes for shallow water models for viscoplastic materials on variable topography (or bathymetry). The associated difficulties are twofold. First, we will place ourselves in a context -increasingly in use- where no regularization method is used and thus the variational inequalities translating the plastic nature of the material are handled directly through duality methods. Second, we will describe new well-balanced schemes in this viscoplastic context to take into account general bottoms and wet/dry fronts.

In recent years, an increasing interest has been developed for shallow water models in the context of simulations for the flow of viscoplastic materials down inclined planes. Viscoplastic materials are characterized by the existence of a yield stress: below a certain critical threshold in the imposed stress, there is no deformation and the material behaves like a rigid solid, but when that yield value is exceeded, the material flows like a fluid. Such flow behaviour can be encountered in many practical situations such as food pastes, heavy oils, lavas and avalanches. As a consequence, the theory of the fluid mechanics of such materials has applications in a wide variety of fields such as chemical industry, energy industry and geophysical fluid dynamics.

From the mathematical viewpoint, the non-linearity associated to viscoplastic models (such as the Bingham model, as we will see below), leads to feasible but very expensive computational times for the full 3D equations (see e.g. [29]). Consequently, numerous reduced 2D model using the shallow flow approximation have been derived. In the context of avalanches, we refer to the article of Ancey [2] and references therein for a detailed review on these developments.

\footnotetext{
This document is an improved version (some misprints corrected) of the published article whose DOI is given below.

${ }^{*}$ Corresponding author

Email addresses: edofer@us .es (Enrique D. Fernández-Nieto), jmgallardo@uma.es (José M. Gallardo), Paul.Vigneaux@math.cnrs.fr (Paul Vigneaux)
} 
Recently, in [26], an interesting shallow water model based on a Bingham-like constitutive law together with Coulomb frictional condition on the bottom was derived, in local coordinates for the case of a non-planar topography. But the algorithm, presented to solve associated equations, does not take into account either well-balanced properties or the treatment of wet/dry fronts. Another shallow model based on the Herschel-Bulkley constitutive law (which generalizes the Bingham law) was derived in [1] and a new well-balanced scheme was introduced to take into account both non-linearities of this constitutive law, leading to a scheme which preserves more accurately stationary states. This point is important when it comes to determine the stopping time of the flow, when the material enters in its rigid state. And this kind of property is also linked to the use of duality methods which allow to properly deal with the plasticity.

Indeed, a common point of an increasing part of the recently developed numerical methods for viscoplastic flows (see e.g. [34], [38, 37], [25], [36], [26]) is that they use decomposition-coordination methods to solve the variational inequality associated to constitutive laws with a so-called plastic threshold (the most simple and iconic one being the Bingham model). This kind of approach takes its roots in the seminal works of Duvaut-Lions [15] and the series of papers of Glowinski and coworkers (see the recent book [23] for a detailed review), initiated at the end of the seventies and anchored in the Augmented Lagrangian formalism. One of the crucial advantage of these methods over regularized approaches (see e.g. Papanastasiou's [30] or the so-called bi-viscosity methods [14]) is that they rigorously take into account the plastic threshold. Of note, it is well known that in Augmented Lagrangian (AL) methods, the optimal values of the parameters are not easy to determine in the general case. These parameters $((r, \rho)$ in Glowinski's nomenclature) influence the speed of convergence of the iterative process towards the saddle-point, solution of the problem. As a consequence, a study of some sort of optimality for such parameters is of real interest when it comes to improve the computational efficiency. As an alternative duality method, one can use the so-called Bermúdez-Moreno (BM) algorithm. This method, which is built upon some properties of the Yosida approximation of maximal monotone operators, has been extensively used for a wide range of applications (see [20] and the references therein). In order to apply the method, the Yosida approximation of the subdifferential associated to the non-differentiable operator appearing in the formulation of the considered model needs to be determined. As for the AL, the performance of the algorithm strongly depends on the choice of two constant parameters. Fortunately, several ways to overcome this problem have been proposed in the literature $([32,31,20])$, and they will be considered in this paper.

Another difficulty that appears when it comes to couple shallow-water models and viscoplastic constitutive laws, is the adequate coupling between the discretization associated to the duality method and the one associated with the spatial terms, in such a way the global scheme is well-balanced. For shallow water type equations, finite volume methods have proved their efficiency and we adopt them in the present work. In this context, a careful treatment must be made to design a well-balanced scheme when coupling the finite volume scheme and the duality method. This idea was first introduced in [8], in the context of a Bingham fluid treated with an AL method. We extend here this idea for a Shallow-Water-Bingham model on a general topography and in the presence of wet/dry fronts.

The well-balanced properties are related to the stationary solutions of the system. In our case, we seek numerical schemes which preserve exactly two types of stationary solutions. For hyperbolic systems with source terms, a discretization of the source terms compatible with the one of the flux term must be performed. Otherwise, stemming from the numerical diffusion terms, a first order error in space takes place. This error, after time iteration, may yield large errors in wave amplitude and speed. The pioneering work by Roe [33] relates the choice of the approximation of the source term with the property of preserving stationary solutions. Bermúdez and Vázquez-Céndón introduced in [5] an extension of Roe's solver, in the context of shallow water equations, which preserves exactly the stationary solution of water at rest. This work originated the so-called well-balanced solvers, in the sense that the discrete source terms balance the discrete flux terms when computed on some (or all) of the steady solutions of the continuous system. Different extensions have been done: see for instance Greenberg and Leroux [24], LeVeque [28], Chacón et al. [12]. An additional difficulty in the simulation of free surface flows comes from the appearance of dry areas in the computational domain, due to the fluid evolution or to the initial conditions. Standard numerical schemes may compute spurious solutions in the presence of wet/dry fronts, unless appropriate modifications are made. See [7, 35] for a review on some methods appearing in the literature to deal with this problem. Moreover, in the context of shallow water equations, Roe-type schemes lose their well-balanced properties when wet/dry transitions appear. Indeed, they may produce nonphysical negative values of the thickness of the water layer near the wet/dry front. Some ways to modify Roe's method to fix these problems have been proposed in $[11,10]$. 
The contribution of this paper is twofold. First, by adapting the guidelines in [31], we determine, in a theoretical way, an optimal choice of parameters in the sense that they provide the highest rate of convergence for the BM algorithm. For the AL, we perform some numerical studies of the optimal choice of parameters and we then compare both methods on various problems to give insight on their respective behaviours. To our best knowledge this is the first time that BM is applied to such kind of models and that such a systematic comparative study of the behaviour of the number of iteration in duality methods is done for several very different viscoplastic flows.

Second, both for the AL and BM methods which are embodied in a general framework, we design a well-balanced scheme which takes into account wet/dry fronts on general bottoms, for such viscoplastic free surface flows. Again, this is the first time that dry area treatment is proposed for flows with plastic behaviour. This is crucial when it comes to study real applications where there are always a flow with wet/dry front and a rigorous treatment is needed to compare qualitatively numerical simulations and physical experiments.

This work is organized as follows. In Section 2, we introduce a typical model for viscoplastic free-surface flows on general bottoms and the general resolution approach. Then in Section 3, we present in detail the two duality methods used to treat the viscoplastic behaviour of the material, namely the AL and the BM methods. In Section 4, we describe the design of the overall well-balanced scheme which takes into account general bottoms and the presence of wet/dry fronts. Numerical tests are finally presented in Section 5 to illustrate the various properties of the scheme and compare both methods. A duct flow test and a stationary test allow to make a convergence analysis thanks to the availability of analytic solutions. Then, several tests of avalanches, academic but very challenging from the numerical viewpoint, are performed to show the robustness of the scheme. Conclusions are drawn in Section 6.

\section{Model and resolution approaches}

As a model problem for viscoplastic shallow flows, we use the Bingham shallow-water model derived in [8], but we add here the fact that the bottom is more general than a plane slope. The derivation being very close, we refer to [8] for more details and here we directly present the resulting model. Its physical characteristics and relevance are briefly described in the following.

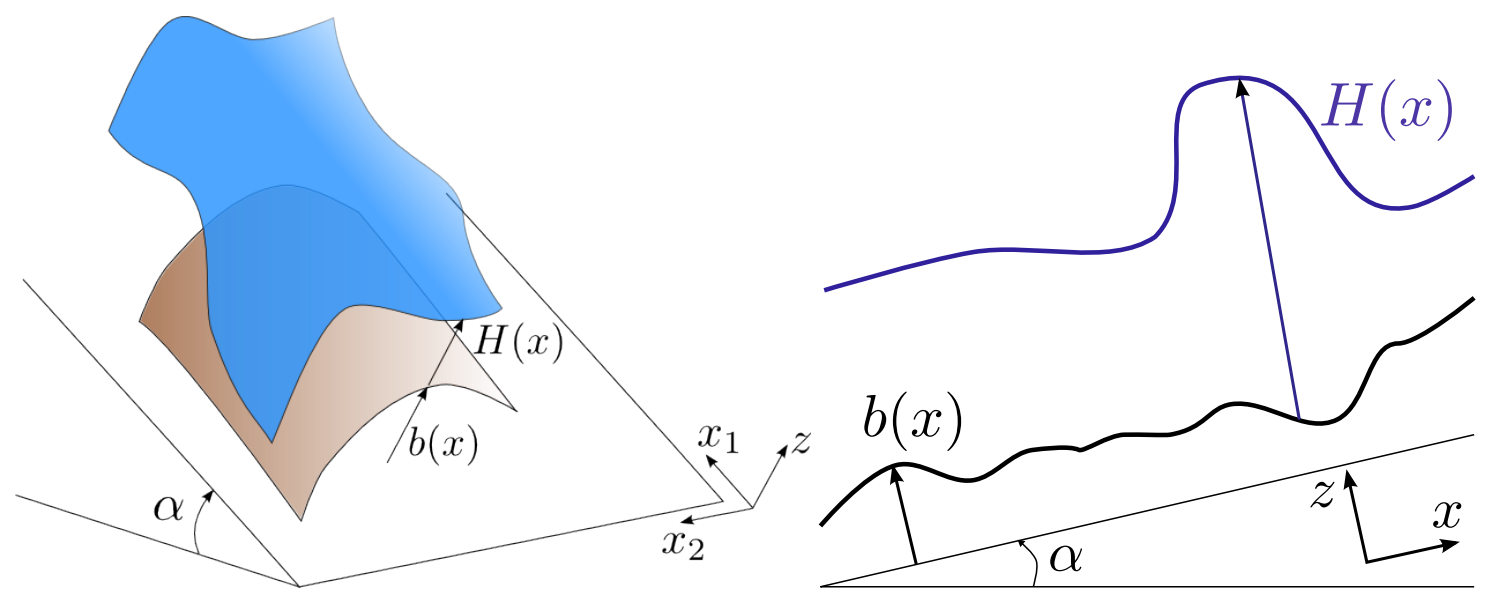

Figure 1: Domain in 2D (left) and 1D (right).

The geometry is as shown on Figure 1 . We consider a fluid domain of height $H$ over a general bottom $b$. More precisely, let $\Omega \subset \mathbb{R}^{2}$ be a given domain for the space variable $x$. The $\mathbb{R}^{2}$ plane generated by $\Omega$ is supposed to be sloping at an angle $\alpha$ from the horizontal plane. We denote by $z \in \mathbb{R}$ the variable in the orthogonal direction to $\Omega$. The 
bottom which bounds the fluid by below is defined by $b(x), x \in \Omega$. We denote by $\mathcal{D}(t)$ the fluid domain defined as

$$
\mathcal{D}(t)=\{(x, z) \in \Omega \times \mathbb{R} / b(x)<z<b(x)+H(t, x)\},
$$

where $H$ is the time-dependent height of the fluid.

As usual for shallow water type models, we denote by $V=V(t, x) \in \mathbb{R}^{2}$ the vector of the average of the velocity (orthogonal to the $z$-axis) along the depth of the fluid (i.e. from $z=b(x)$ to $z=b(x)+H(t, x)$ ). We take into account the fact that there may be friction on the bottom through a coefficient $\beta$. The fluid undergoes a body force denoted as $\left(f_{\Omega}, f_{z}\right) \in \mathbb{R}^{2} \times \mathbb{R}$ in the $\Omega \times z$ frame of reference. Note that $f_{\Omega}$ and $f_{z}$ are both assumed to be constant.

Since we are considering a Bingham constitutive law, the material is characterized by a viscosity $\eta$ and a yield stress $\tau_{y}$. The latter is associated to the plastic behaviour of the material and this leads (cf. [15]) to a variational inequality for the momentum conservation relation (see equation 4). On the contrary, the conservation of mass is rather classic for this type of integrated model (see equation 3). Given the space

$$
\mathcal{V}(t)=\left\{\Psi \in H^{1}(\Omega)^{2} / \Psi=0 \text { on } \partial \Omega\right\}:=H_{0}^{1}(\Omega)^{2}
$$

and some initial conditions at $t=0$, the problem is to find $H \in L^{2}\left([0, T], L^{\infty}(\Omega)\right), V \in L^{2}([0, T] ; \mathcal{V}(t))$, with $\partial_{t} V \in$ $L^{2}\left([0, T] ; L^{2}(\Omega)^{2}\right)$, such that

$$
\partial_{t} H+\operatorname{div}_{x}(H V)=0
$$

and

$$
\begin{aligned}
& \forall \Psi \in \mathcal{V}(t), \quad \int_{\Omega} H\left(\partial_{t} V \cdot(\Psi-V)+V \cdot \nabla_{x} V(\Psi-V)\right) d x+\int_{\Omega} \beta V \cdot(\Psi-V) d x \\
& +\int_{\Omega} 2 \eta H D(V): D(\Psi-V) d x+\int_{\Omega} 2 \eta H \operatorname{div}_{x} V\left(\operatorname{div}_{x} \Psi-\operatorname{div}_{x} V\right) d x \\
& +\int_{\Omega} \tau_{y} H\left(\sqrt{|D(\Psi)|^{2}+\left(\operatorname{div}_{x} \Psi\right)^{2}}-\sqrt{|D(V)|^{2}+\left(\operatorname{div}_{x} V\right)^{2}}\right) d x \\
& \geq \int_{\Omega} H\left(f_{\Omega}+f_{z} \nabla_{x} b\right) \cdot(\Psi-V)-\int_{\Omega} \frac{(H)^{2}}{2} f_{z}\left(\operatorname{div}_{x} \Psi-\operatorname{div}_{x} V\right) d x,
\end{aligned}
$$

where

$$
\begin{gathered}
D(U):=\frac{1}{2}\left[\nabla_{x} U+\left(\nabla_{x} U\right)^{t}\right], \\
\nabla_{x} U:=\left(\frac{\partial U_{i}}{\partial x_{j}}\right)_{i, j}, \quad i=1,2, j=1,2, \\
\operatorname{div}_{x} U:=\frac{\partial U_{1}}{\partial x_{1}}+\frac{\partial U_{2}}{\partial x_{2}}, \quad \forall U(t, \cdot):=\left(U_{1}, U_{2}\right) \in \mathcal{V}(t) .
\end{gathered}
$$

Of note, usual Sobolev embeddings and the fact that $H$ is bounded allow aforementioned problem to be well defined. As said previously, this model is based on a so-called shallow water approximation, i.e. the height of the fluid is assumed to be much smaller than the characteristic length of the domain. Furthermore, this model was derived through an asymptotic expansion where the slope is supposed to be small $(\alpha \ll 1)$ and the norm of the gradient of $b(x)$ is small $\left(\left\|\nabla_{x} b\right\| \ll 1\right)$.

But it is worth noting that this model is also valid for a slope $\alpha=0$ (horizontal bottom), which is not generally the case for other models proposed in the literature (see for example [3], [18]).

Another interesting feature of the model is that in the case of a plane horizontal slope $(\alpha=0)$ and with a vanishing yield stress $\left(\tau_{y}=0\right)$, we recover a viscous shallow water system which has the same structure as the one derived by Gerbeau and Perthame in [21] (note that the hypothesis of friction at the bottom, instead of a no-slip condition is a key point in this degeneracy to [21]). The shallow water formulation (4) is in weak form. It can be rewritten in the strong form to have the expression of the associated (integrated) Bingham constitutive law. Namely, the corresponding formulation is

$$
H\left(\partial_{t} V+V \cdot \nabla_{x} V\right)+\beta V-\operatorname{div}_{x}(H \sigma)=H\left(f_{\Omega}+f_{z} \nabla_{x} b\right)-\nabla_{x}\left(\frac{(H)^{2}}{2} f_{z}\right),
$$


where

$$
\begin{cases}\sigma=2 \eta(D(V)+\operatorname{tr}(D(V)) I)+\tau_{y} \frac{D(V)+\operatorname{tr}(D(V)) I}{\sqrt{|D(V)|^{2}+|\operatorname{tr}(D(V))|^{2}}} & \text { if }|D(V)| \neq 0 \\ |\sigma| \leq \tau_{y} & \text { if }|D(V)|=0,\end{cases}
$$

the second invariant $|\sigma|$ of a tensor $\sigma$ being defined here as

$$
|\sigma|:=\sqrt{\Sigma_{i, j} \sigma_{i j}^{2}} .
$$

Note that in the following, the body force will be the influence of gravity, denoted by $g$. To write this force, we must decide what is the orientation of the plane generated by $\Omega$; by convention we will say that if $\left(x_{1}, x_{2}, z\right)$ is the frame of reference (cf. Figure 1-a), then the tilted axis (with respect to the horizontal) is $x_{1}$, i.e.

$$
f_{\Omega}=(-g \sin \alpha, 0), \quad f_{z}=-g \cos \alpha .
$$

In this paper, we will fulfill our objectives on the 1D version of (2)-(3)-(4). Of note, the ideas presented here can be extended in 2D. This extension being not trivial, it will be presented in another article [17]. In the 1D case, we naturally take $x \in \Omega=[0, L]$ and the associated frame of reference becomes $(x, z)$ (cf. Figure 1-b). We have then

$$
\mathcal{V}(t)=\left\{\Psi \in H^{1}(\Omega) / \Psi=0 \text { on } \partial \Omega\right\}:=H_{0}^{1}([0, L]),
$$

and the problem (3)-(4) degenerates to: find $H \in L^{2}\left([0, T], L^{\infty}([0, L])\right)$ and $V \in L^{2}([0, T], \mathcal{V}(t))$, with $\partial_{t} V \in$ $L^{2}\left([0, T], L^{2}([0, L])\right)$, such that

$$
\partial_{t} H+\partial_{x}(H V)=0,
$$

and

$$
\begin{aligned}
& \forall \Psi \in \mathcal{V}(t), \quad \int_{0}^{L} H\left(\partial_{t} V(\Psi-V)+\frac{1}{2} \partial_{x}\left(V^{2}\right)(\Psi-V)\right) d x+\int_{0}^{L} 4 \eta H \partial_{x} V \partial_{x}(\Psi-V) d x \\
& +\int_{0}^{L} \tau_{y} \sqrt{2} H\left(\left|\partial_{x} \Psi\right|-\left|\partial_{x} V\right|\right) d x+\int_{0}^{L} \beta V(\Psi-V) d x \\
& \geq \int_{0}^{L} H\left(f_{\Omega}+f_{z} \partial_{x} b\right)(\Psi-V) d x-\int_{0}^{L} \frac{H^{2}}{2} f_{z}\left(\partial_{x} \Psi-\partial_{x} V\right) d x .
\end{aligned}
$$

The gravity becomes (note that, as the velocity, the projection of the force on $\Omega$ is now a scalar):

$$
f_{\Omega}=-g \sin \alpha, \quad f_{z}=-g \cos \alpha .
$$

In this paper, as in many schemes used in the literature for this type of models, we will consider a first order backward semi-discretization in time (explicit Euler's method). We denote the time step by $\Delta t$. We have:

$$
\frac{H^{n+1}-H^{n}}{\Delta t}+\partial_{x}\left(H^{n} V^{n}\right)=0,
$$

and

$$
\begin{aligned}
& \int_{0}^{L} H^{n}\left(\frac{V^{n+1}-V^{n}}{\Delta t}\left(\Psi-V^{n+1}\right)+\frac{1}{2} \partial_{x}\left(\left(V^{n}\right)^{2}\right)\left(\Psi-V^{n+1}\right)\right) d x+\int_{0}^{L} \beta V^{n+1}\left(\Psi-V^{n+1}\right) d x \\
& +\int_{0}^{L} \tau_{y} \sqrt{2} H^{n}\left(\left|\partial_{x} \Psi\right|-\left|\partial_{x} V^{n+1}\right|\right) d x+\int_{0}^{L} 4 \eta H^{n} \partial_{x}\left(V^{n+1}\right) \partial_{x}\left(\Psi-V^{n+1}\right) d x \\
& \geq \int_{0}^{L} H^{n}\left(f_{\Omega}+f_{z} \partial_{x} b\right)\left(\Psi-V^{n+1}\right) d x-\int_{0}^{L} \frac{\left(H^{n}\right)^{2}}{2} f_{z}\left(\partial_{x} \Psi-\partial_{x} V^{n+1}\right) d x, \quad \forall \Psi .
\end{aligned}
$$


Doing so, we see that problem on the height and problem on the velocity are decoupled. At each time step, supposing that we know $\left(H^{n}, V^{n}\right)$, we need to solve both problems for $\left(H^{n+1}, V^{n+1}\right)$.

One of the goals of this article is to compare two duality methods to handle the variational inequality of the problem on the velocity, namely the Augmented Lagrangian method and Bermúdez-Moreno method. It is the subject of the next section, where we will also see more clearly the underlying shallow water nature of this system. For this kind of equations, finite volume discretizations are particularly well suited and this is why we want to use them in this context. The other goal of this paper is to show that a careful design of the scheme is needed to obtain a wellbalanced property in the case of variable bottom and in the presence of wet/dry fronts. This will be treated in Section 4.

\section{Treating the velocity inequality with two duality methods}

The speed problem of the above shallow Bingham model is a variational inequality and consequently specific methods to solve it are needed. Various types of methods exist in the literature and, in this paper, we choose two of them which have proved to be efficient for such problems (see e.g. [27]), namely the Augmented Lagrangian method and the Bermúdez-Moreno method. Their definitions and derivations are different, but interestingly the obtained structure of the algorithms is the same. We will thus make the most of this fact in Section 4, to design a unified scheme for the space discretization. In addition, this common structure in terms of code implementation makes it interesting to compare their efficiency and computational cost. In particular, both algorithms depend on a parameter which influence the speed of convergence to the solution. Consequently, it is interesting to determine if optimal parameters can be derived theoretically.

The Augmented Lagrangian algorithm is a method which has been extensively used for a wide range of non-linear optimization problems (see [22]). It appears that (17) can be reformulated as a minimization problem and solving for $V^{n+1}$ ends up to find the saddle-point of an Augmented Lagrangian. This approach allows to elegantly deal with the non-differentiable terms of (17).

For sake of brevity, the full derivation of the Augmented Lagrangian algorithm for (17) is given in Appendix A. Of note, the final form of the present Augmented Lagrangian algorithm has a structure which is very similar to the one derived the following subsection and Appendix A is also here for completeness in comparing the two approaches.

Concerning the so-called augmentation parameter $r$, it is generally difficult to find a priori an optimal value which leads to the fastest convergence of this iterative method. Some optimal parameters are derived in [19] for very simple model problem and, in the context of seismic reflection tomography, a heuristics was proposed to evolve $r$ along the iterations of the AL to increase the speed of the algorithm [13]. But overall, a general methodology to find theoretically an optimal value of $r$ seems to remain an open question. This fact was one of the reasons to study in more details another duality method, namely the Bermúdez-Moreno method.

\subsection{Study of the BM approach}

For the sake of brevity, the full derivation of the Bermúdez-Moreno method is given in Appendix B and we will directly give the resulting algorithm in the following. It is important to note that it is as easy to implement as the Augmented Lagrangian algorithm.

\subsubsection{The BM algorithm}

Let us summarize the BM algorithm for the speed problem (17).

\section{Bermúdez-Moreno algorithm}

- Initialization: suppose that $V^{n}, H^{n}$ and $\theta^{n}$ are known. For $k=0$, we set $V^{k}=V^{n}$ and $\theta^{k}=\theta^{n}$.

- Define the parameters $\lambda$ and $\omega$ (See Section 3.1.2).

- Iterate: 
- Find $V^{k+1} \in \mathcal{V}$ solution of the following linear problem:

$$
\begin{aligned}
& \left(\frac{H^{n}}{\Delta t}+\beta\right) V^{k+1}-\partial_{x}\left(\left(4 \eta H^{n}+\omega\right) \partial_{x} V^{k+1}\right)-\partial_{x}\left(\omega \partial_{x} V^{k+1}\right) \\
= & \frac{H^{n}}{\Delta t} V^{n}-\frac{H^{n}}{2} \partial_{x}\left(\left(V^{n}\right)^{2}\right)+\frac{1}{2} \partial_{x}\left(\left(H^{n}\right)^{2} f_{z}\right)+H^{n}\left(f_{\Omega}+f_{z} \partial_{x} b\right)+\partial_{x} \theta^{k} .
\end{aligned}
$$

- Update the so-called Bermúdez-Moreno multiplier $\theta^{k+1}$ via $\xi^{k+1}=\partial_{x} V^{k+1}+\lambda \theta^{k}$ and

$$
\theta^{k+1}= \begin{cases}\frac{-\omega \xi^{k+1}+\tau_{y} \sqrt{2} H^{n}(x)}{1-\lambda \omega} & \text { if } \xi^{k+1}>\lambda \tau_{y} \sqrt{2} H^{n}(x), \\ \frac{\xi^{k+1}}{\lambda} & \text { if } \xi^{k+1} \in\left[-\lambda \tau_{y} \sqrt{2} H^{n}(x), \lambda \tau_{y} \sqrt{2} H^{n}(x)\right], \\ \frac{-\omega \xi^{k+1}-\tau_{y} \sqrt{2} H^{n}(x)}{1-\lambda \omega} & \text { if } \xi^{k+1}<-\lambda \tau_{y} \sqrt{2} H^{n}(x) .\end{cases}
$$

Note that this computation is local in space, i.e., it is done at each discretization point.

- Check convergence (see below) and update: $V^{k}=V^{k+1}, \theta^{k}=\theta^{k+1}, k \mapsto k+1$ and go to the next iteration...

- ... until convergence is reached:

$$
\frac{\left\|\theta^{k+1}-\theta^{k}\right\|}{\left\|\theta^{k}\right\|} \leq \text { tol. }
$$

At convergence, we get the value of $V^{n+1}$ by setting $V^{n+1}=V^{k+1}$ (in the numerical tests presented in this paper, we set tol $=10^{-5}$ ).

Up to now, we did not describe the discretization in space. As we said previously, we want to adopt a finite volume approach. Consequently, it is worth realizing that the underlying global problem coupling (16) and (17) involves the following system (we use a slight change of notation which will be useful in the following: $H^{n+1}$ is denoted as $H^{k+1}$; in spite of this choice, note again that $H^{k+1}$ is not involved in the Bermúdez-Moreno algorithm and, so, does not change in this loop):

$$
(\tilde{P})^{n, k}\left\{\begin{array}{l}
\frac{H^{k+1}-H^{n}}{\Delta t}+\partial_{x}\left(H^{n} V^{n}\right)=0 \\
H^{n}\left(\frac{V^{k+1}-V^{n}}{\Delta t}\right)+\beta V^{k+1}-\partial_{x}\left(4 \eta H^{n} \partial_{x}\left(V^{k+1}\right)\right)-\partial_{x}\left(\omega \partial_{x} V^{k+1}\right) \\
=H^{n}\left(f_{\Omega}+f_{z} \partial_{x} b\right)-\frac{H^{n}}{2} \partial_{x}\left(\left(V^{n}\right)^{2}\right)+\frac{1}{2} \partial_{x}\left(\left(H^{n}\right)^{2} f_{z}\right)+\partial_{x} \theta^{k} .
\end{array}\right.
$$

Consequently, even if there is a decoupling of both problems in terms of the time discretization and the BermúdezMoreno algorithm, it appears that to obtain a global well-balanced scheme, there must be a coupling between the mass and momentum equations induced by the source terms (involving topography and the multiplier $\theta$ ). For shallow water type systems with source terms, this has been extensively studied in the literature. In Section 4, we precisely describe the aforementioned coupling for the present problem.

\subsubsection{Study of the optimal parameter}

As it has been documented in the literature, the main drawback of the BM algorithm is that its rate of convergence strongly depends on the choice of parameters $\lambda$ and $\omega$. Several efforts have been made to overcome this problem, allowing the choice of appropriate parameters in different functional frameworks ([31], [32], [20]). We will adapt here the guidelines in [31] in order to deduce an optimal choice of parameters, the main idea being to look for $\omega$ minimizing the contractivity constant of the sequence $\theta^{k}-\theta$. The condition

$$
\lambda \omega=\frac{1}{2}
$$


will be assumed throughout the rest of the paper, so only one of the parameters has to be chosen, say $\omega$. The interested reader will find all the details of the derivation in Appendix C. To sum up, a quasi-optimal choice of the parameter $\omega$ would be given by

$$
\omega_{\mathrm{opt}}\left(H_{\max }^{n}\right)=\left(\frac{H_{\max }^{n}}{\Delta t}+\beta\right) \frac{L^{2}}{N \pi^{2}}+4 \eta H_{\max }^{n}
$$

where $H_{\max }^{n}=\left\|H^{n}\right\|_{\infty}$.

\subsubsection{Some remarks on the treatment of wet/dry fronts}

Although not explicitly stated, in the preceding sections it has been assumed that $H^{n}(x) \geq H_{\min }^{n}$ for a certain constant $H_{\min }^{n}>0$, which means that there is no dry area in the computational domain. Following [31, 32], one possible way to take into account the appearance of dry areas is to extend the function $\Phi$ ( $c f$. Appendix $\mathrm{C}$ ) as follows:

$$
\Phi(x, z)= \begin{cases}\tau_{y} \sqrt{2} H^{n}(x)|z| & \text { if } H^{n}(x) \geq 0, \\ +\infty & \text { otherwise. }\end{cases}
$$

In this case, a simple computation shows that the expression (B.6) for the Yosida approximation $G_{\lambda}^{\omega}$ remains valid, including the case $H^{n}(x)=0$. Therefore, the BM algorithm in Section 3.1.1 can be applied in the presence of wet/dry fronts, after taking into account the modifications to be proposed in Section 4.

On the other hand, as $H^{n}$ approaches to zero the coercivity constant (B.2) also tends to zero, thus degrading the convergence of the BM method. Moreover, when looking for the optimal value of the parameter $\omega$, the contractivity constant $\mathcal{L}(\omega)$ appearing in (C.7) is close to one. It has been verified numerically that, if no modification is made, the convergence of the BM algorithm is considerably slower in the presence of wet/dry fronts. Fortunately, this problem can be overcome by taking the parameter $\omega$ depending on $H^{n}(x)$, following the guidelines in [32]. After extensive numerical investigation, we have found that the best convergence results are obtained for

$$
\omega_{\mathrm{opt}}\left(H^{n}(x), H_{\max }^{n}\right)=\varpi_{\mathrm{opt}}\left(H_{\max }^{n}\right) H^{n}(x),
$$

with

$$
\varpi_{\mathrm{opt}}\left(H_{\max }^{n}\right)=\left(\frac{1}{\Delta t}+\frac{\beta}{H_{\max }^{n}}\right) \frac{L_{\mathrm{w}}^{2}}{N \pi^{2}}+4 \eta,
$$

where $L_{\mathrm{w}}$ is the length of the wet domain. As it is shown in Test 5.2, in this case the speed of convergence of the BM algorithm is greatly improved.

\section{Well-balanced discretization with general bed and wet/dry fronts}

As said previously, either for AL and BM methods, we need now to describe how to perform the discretization in space. This point is essentially inspired by finite volume methods for shallow water type systems. Since the structure of the resulting systems are very close, we will try to unify the description as much as possible and point out when necessary the adaptation needed for each case.

The space domain $[0, L]$ is divided in computing cells $I_{i}=\left[x_{i-1 / 2}, x_{i+1 / 2}\right]$. For simplicity, we suppose that these cells have a constant size $\Delta x$. Let us define $x_{i+\frac{1}{2}}=(i+1 / 2) \Delta x$ and $x_{i}=i \Delta x$, the center of the cell $I_{i}$. We define $W^{k+1}$ (thanks to the aforementioned cosmetic harmonization of the notation) as the following vector of the unknowns of problem $(P)^{n, k}\left(\right.$ or $\left.(\tilde{P})^{n, k}\right)$,

$$
W^{k+1}(x)=\left[H^{k+1}(x), V^{k+1}(x)\right] .
$$

The two equations (A.8) of problem $(P)^{n, k}$, as well as the two equations (21) of problem $(\tilde{P})^{n, k}$ can be rewritten under the form:

$$
\begin{gathered}
D\left(W^{n}\right)\left(\frac{W^{k+1}-W^{n}}{\Delta t}+\partial_{x} F\left(W^{n}\right)\right)-\partial_{x}\left(\left(4 \eta H^{n}+\delta^{n}\right) \underline{I} \partial_{x} W^{k+1}\right) \\
=-\beta \underline{I} W^{k+1}+S\left(W^{n}\right) \partial_{x} \bar{\sigma}^{k}
\end{gathered}
$$


where

$$
\begin{gathered}
D\left(W^{n}\right)=\left(\begin{array}{cc}
1 & 0 \\
0 & H^{n}
\end{array}\right), \quad F\left(W^{n}\right)=\left(\begin{array}{c}
H^{n} V^{n} \\
\frac{\left(V^{n}\right)^{2}}{2}-f_{z} H^{n}
\end{array}\right), \quad \underline{I}=\left(\begin{array}{ll}
0 & 0 \\
0 & 1
\end{array}\right), \\
S\left(W^{n}\right)=\left(\begin{array}{cc}
0 & 0 \\
H^{n} & 1
\end{array}\right), \quad \bar{\sigma}^{k}=\left(\begin{array}{c}
f_{\Omega} x+f_{z} b \\
\zeta^{k}
\end{array}\right) .
\end{gathered}
$$

The definition for $\delta^{n}$ and $\zeta^{k}$ depends on the duality method. Namely,

- for the AL method:

$$
\delta^{n}=r H^{n}, \quad \zeta^{k}=H^{n}\left(\mu^{k}-r q^{k}\right)
$$

- for the BM method:

$$
\delta^{n}=\omega^{n}, \quad \zeta^{k}=\theta^{k},
$$

where $\omega^{n}$ is defined by the optimal value in terms of $H^{n}$ (see Subsections 3.1.2 and 3.1.3).

We denote by $W_{i}^{k+1}$ the approximation of the cell average of the exact solution provided by the numerical scheme:

$$
W_{i}^{k+1} \cong \frac{1}{\Delta x} \int_{x_{i-1 / 2}}^{x_{i+1 / 2}} W^{k+1}(x) d x .
$$

Furthermore, $\theta, \mu$ and $q$ are approximated at the center of the dual mesh: $\theta_{i+1 / 2}^{k}, \mu_{i+1 / 2}^{k}$ and $q_{i+1 / 2}^{k}$ are approximations of $\theta^{k}\left(x_{i+1 / 2}\right), \mu^{k}\left(x_{i+1 / 2}\right)$ and $q^{k}\left(x_{i+1 / 2}\right)$, respectively. Consequently, we define

$$
\zeta_{i+1 / 2}^{k}= \begin{cases}H_{i+1 / 2}^{n}\left(\mu_{i+1 / 2}^{k}-r q_{i+1 / 2}^{k}\right) & \text { in the case of AL }(a) \\ \theta_{i+1 / 2}^{k} & \text { in the case of } \operatorname{BM}(b)\end{cases}
$$

and

$$
\delta_{i+1 / 2}^{n}= \begin{cases}r H_{i+1 / 2}^{n} & \text { in the case of } \operatorname{AL}(a) \\ \omega_{i+1 / 2}^{n} & \text { in the case of } \operatorname{BM}(b)\end{cases}
$$

For the BM algorithm $\omega_{i+1 / 2}^{n}$ is defined in terms of $\omega_{\text {opt }}$, following (22) or (23). Concretely, we can set

$$
\omega_{i+1 / 2}^{n}=\omega_{\mathrm{opt}}\left(\max _{i}\left(H_{i}^{n}\right)\right),
$$

where $\omega_{\mathrm{opt}}(H)$ is defined by (22). Following (23) we can also set the following definition:

$$
\omega_{i+1 / 2}^{n}=\varpi_{\mathrm{opt}}\left(\max _{i}\left(H_{i}^{n}\right)\right) H_{i+1 / 2}^{n},
$$

where $\varpi_{\mathrm{opt}}($.$) is defined by (24) and$

$$
H_{i+1 / 2}^{n}=\frac{H_{i}^{n}+H_{i+1}^{n}}{2}
$$

We also define $\lambda_{i+1 / 2}^{n}$ in terms of $\omega_{i+1 / 2}^{n}$ :

$$
\lambda_{i+1 / 2}^{n} \omega_{i+1 / 2}^{n}=\frac{1}{2} .
$$

As mentioned during the presentation of both duality methods, we can suppose that the values $W_{i}^{k}, W_{i}^{0}=\left[H^{n}, V^{n}\right]$ and $\zeta_{i+1 / 2}^{k}$ are known for all $i$. Then, we proceed as follows.

System (25) is discretized as

$$
D\left(W_{i}^{n}\right)\left(\frac{W_{i}^{k+1}-W_{i}^{n}}{\Delta t}+\frac{\phi\left(W_{i}^{n}, W_{i+1}^{n},\left\{\zeta_{j+1 / 2}^{k}\right\}_{j=i-1}^{j=i+1}\right)-\phi\left(W_{i-1}^{n}, W_{i}^{n},\left\{\zeta_{j+1 / 2}^{k}\right\}_{j=i-2}^{j=i}\right)}{\Delta x}\right)
$$




$$
\begin{gathered}
-\frac{1}{\Delta x^{2}}\left(\left(4 \eta H_{i+1 / 2}^{n}+\delta_{i+1 / 2}^{n}\right) \underline{I}\left(W_{i+1}^{k+1}-W_{i}^{k+1}\right)-\left(4 \eta H_{i-1 / 2}^{n}+\delta_{i-1 / 2}^{n}\right) \underline{I}\left(W_{i}^{k+1}-W_{i-1}^{k+1}\right)\right) \\
=-\beta \underline{I} W_{i}^{k+1}+S\left(W_{i}^{n}\right) \frac{\bar{\sigma}_{i+1 / 2}^{k}-\bar{\sigma}_{i-1 / 2}^{k}}{\Delta x} .
\end{gathered}
$$

The definition of $\bar{\sigma}_{i+1 / 2}^{k}$ is

$$
\bar{\sigma}_{i+1 / 2}^{k}=\left(\begin{array}{c}
f_{\Omega} x_{i+1 / 2}+f_{z} \frac{b_{i}+b_{i+1}}{2} \\
\zeta_{i+1 / 2}^{k}
\end{array}\right) .
$$

Finally, $\phi\left(W_{i}^{n}, W_{i+1}^{n},\left\{\zeta_{j+1 / 2}^{k}\right\}_{j=i-1}^{j=i+1}\right)$ is a numerical flux function, approximation of $F\left(W^{n}\right)$ at $x_{i+1 / 2}$.

In order to complete the numerical scheme, we must precise the definition of $\phi$. We consider a family of numerical flux functions which define a well-balanced finite volume solver. System (25) can be seen as a semi-discretization in time of a parabolic system, which for $\eta=\delta=0$ degenerates into a hyperbolic system with source terms. Following [12], in order to obtain a well-balanced finite volume method, the numerical flux $\phi$, approaching the flux function $F(W)$ at $x_{i+1 / 2}$, must depend on the definition of the source terms.

Namely, we consider the following class of numerical flux functions:

$$
\phi\left(W_{i}^{n}, W_{i+1}^{n},\left\{\zeta_{j+1 / 2}^{k}\right\}_{j=i-1}^{j=i+1}\right)=\frac{F\left(W_{i}^{n}\right)+F\left(W_{i+1}^{n}\right)}{2}-\frac{1}{2} Q_{i+1 / 2}^{n}\left(W_{i+1}^{n}-W_{i}^{n}+\mathcal{G}^{n}\left(\left\{\zeta_{j+1 / 2}^{k}\right\}_{j=i-1}^{j=i+1}\right)\right)
$$

where $Q_{i+1 / 2}^{n}$ is the numerical viscosity matrix which particularizes the numerical solver and $\mathcal{G}^{n}\left(\left\{\zeta_{j+1 / 2}^{k}\right\}_{j=i-1}^{j=i+1}\right)$ is a term designed to obtain a well-balanced finite volume method.

The numerical viscosity matrix can be defined in terms of the eigenvalues of the Roe matrix associated to the flux $F(W)$. Let us denote by $\mathcal{A}_{i+1 / 2}^{n}$ the Roe matrix verifying,

$$
F\left(W_{i+1}^{n}\right)-F\left(W_{i}^{n}\right)=\mathcal{A}_{i+1 / 2}^{n}\left(W_{i+1}^{n}-W_{i}^{n}\right) .
$$

This matrix can be diagonalized and its eigenvalues are

$$
\Lambda_{1, i+1 / 2}^{n}=\tilde{V}_{i+1 / 2}^{n}-\sqrt{-f_{z} H_{i+1 / 2}^{n}}, \quad \Lambda_{2, i+1 / 2}^{n}=\tilde{V}_{i+1 / 2}^{n}+\sqrt{-f_{z} H_{i+1 / 2}^{n}},
$$

where $\tilde{V}_{i+1 / 2}^{n}=\left(\sqrt{H_{i}^{n}} V_{i}^{n}+\sqrt{H_{i+1}^{n}} V_{i+1}^{n}\right) /\left(\sqrt{H_{i}^{n}}+\sqrt{H_{i+1}^{n}}\right)$.

In this work we consider Rusanov's method, defined by $Q_{i+1 / 2}^{n}=\alpha_{0, i+1 / 2} I$ with $\alpha_{0, i+1 / 2}=\max \left(\left|\Lambda_{1, i+1 / 2}^{n}\right|,\left|\Lambda_{2, i+1 / 2}^{n}\right|\right)$. As discussed in [1], using a diagonal viscosity matrix allows us to design an algorithm where in the first step we compute the flux associated to the velocity, in the second step we perform an iterative algorithm, and finally we compute the flux associated to the height evolution. As a consequence, in the iterative process of the duality method it is not necessary to recompute the numerical fluxes at each step. See [1] for more details on this discussion. Some other numerical solvers defined in terms of a diagonal viscosity matrix are the Lax-Friedrichs method, corresponding to $Q_{i+1 / 2}^{n}=\frac{\Delta x}{\Delta t} I$, and the modified Lax-Friedrichs method, corresponding to $Q_{i+1 / 2}^{n}=\gamma \frac{\Delta x}{\Delta t} I$, where $\gamma \in(0,1]$ is the CFL number.

As we are considering explicit finite volume solvers, a CFL condition must be imposed to compute the time step. If we consider some of these three methods then $\Delta t$ is computed by imposing the following restriction:

$$
\frac{\Delta t}{\Delta x} \max _{i}\left(\left|\Lambda_{j, i+1 / 2}^{n}\right|, j=1,2\right)=\gamma, \quad \text { with } \quad \gamma \in(0,1] .
$$

For all numerical tests presented in Section 5, we set $\gamma=0.9$.

In the following points we propose:

i) the correction term $\mathcal{G}^{n}\left(\left\{\zeta_{j+1 / 2}^{k}\right\}_{j=i-1}^{j=i+1}\right)$ associated to the well-balancing;

ii) and a numerical treatment that is applied in the case of wet/dry fronts. 


\section{i) Well-balanced correction}

We must describe the term $\mathcal{G}^{n}\left(\left\{\zeta_{j+1 / 2}^{k}\right\}_{j=i-1}^{j=i+1}\right)$ in order to complete the numerical flux function (31). The definition of $\mathcal{G}^{n}$ is related to the well-balanced properties of the numerical scheme. We propose the following definition:

$$
\mathcal{G}^{n}\left(\left\{\zeta_{j+1 / 2}^{k}\right\}_{j=i-1}^{j=i+1}\right)=\frac{1}{f_{z}}\left(\begin{array}{c}
f_{\Omega} \Delta x+f_{z}\left(b_{i+1}-b_{i}\right)+\frac{\Delta\left(\zeta+\delta^{n} \partial_{x} V\right)_{i+1 / 2}^{k}}{H_{i+1 / 2}} \\
0
\end{array}\right),
$$

where $\Delta\left(\zeta+\delta^{n} \partial_{x} V\right)_{i+1 / 2}^{k} / \Delta x$ is an approximation of $\partial_{x}\left(\zeta+\delta^{n} \partial_{x} V\right)^{k}$ at $x_{i+1 / 2}$.

Remark that at convergence of the Augmented Lagrangian loop, $\left(\zeta+\delta^{n} \partial_{x} V\right)$ can be approximated by $H^{n} \mu$, since at this point $q \approx \partial_{x} V$.

We propose the following definition of $\Delta\left(\zeta+\delta^{n} \partial_{x} V\right)_{i+1 / 2}^{k}$, based on a convex combination, by using a flux limiter function, of a second order approximation and a first order one:

$$
\Delta\left(\zeta+\delta^{n} \partial_{x} V\right)_{i+1 / 2}^{k}=\mathcal{D}\left(d_{l}, d_{c}, d_{r}, s_{-1}, s_{0}, s_{1}, s_{2}\right),
$$

with

$$
\begin{gathered}
d_{l}=\zeta_{i-1 / 2}^{k}+\delta_{i-1 / 2}^{n} \frac{V_{i}^{k}-V_{i-1}^{k}}{\Delta x}, \quad d_{c}=\zeta_{i+1 / 2}^{k}+\delta_{i+1 / 2}^{n} \frac{V_{i+1}^{k}-V_{i}^{k}}{\Delta x}, \\
d_{r}=\zeta_{i+3 / 2}^{k}+\delta_{i+3 / 2}^{n} \frac{V_{i+2}^{k}-V_{i+1}^{k}}{\Delta x}, \quad s_{j}=H_{i+j}^{n}+b_{i+j}, \quad j=-1,0,1,2 .
\end{gathered}
$$

The function $\mathcal{D} / \Delta x$ is defined by a combination of a second order approximation of $\partial_{x}\left(\zeta+\delta^{n} \partial_{x} V\right)$ at $x=x_{i+1 / 2}$ with a first order one, by means of a flux limiter function. We propose the following definition:

$$
\mathcal{D}\left(d_{l}, d_{c}, d_{r}, s_{-1}, s_{0}, s_{1}, s_{2}\right)=\chi \frac{d_{r}-d_{l}}{2}+(1-\chi) \overline{\Delta d}_{1},
$$

with

$$
\overline{\Delta d}_{1}= \begin{cases}d_{c}-d_{l} & \text { if } s_{0}<s_{1}, \\ d_{r}-d_{c} & \text { if } s_{0}>s_{1}, \\ \left(d_{r}-d_{l}\right) / 2 & \text { if } s_{0}=s_{1} .\end{cases}
$$

Remark that one of the difficulties of the 2D problem is the definition of the flux limiter $\chi$ and a proper definition of $\mathcal{D}\left(d_{l}, d_{c}, d_{r}, s_{-1}, s_{0}, s_{1}, s_{2}\right)$ allowing to recover the second order well-balanced properties. These will be treated in a forthcoming paper.

The term $\chi=\chi\left(v\left(s_{-1}, s_{0}, s_{1}, s_{2}\right)\right)$ is a flux limiter function with $v\left(s_{-1}, s_{0}, s_{1}, s_{2}\right) \in[0,1]$. We propose to define

$$
v=\max (0, \min (1, \tilde{v})), \quad \tilde{v}= \begin{cases}\frac{3\left(s_{0}-s_{-1}\right)}{s_{2}-s_{-1}}, & \text { if } s_{1}>s_{0}, \\ \frac{3\left(s_{2}-s_{1}\right)}{s_{2}-s_{-1}}, & \text { if } s_{1}<s_{0}, \\ 1 & \text { if } s_{1}=s_{0} \text { or } s_{2}=s_{-1},\end{cases}
$$

and the following definition of the flux limiter function:

$$
\chi(v)=1-\left(1-v^{1 / 4}\right)^{4} .
$$

The definition of this limiter is driven by the fact that we want to put a stronger weight on the second order approximation $\left(\frac{d_{r}-d_{l}}{2}\right)$ compared to the first order one $\left(\overline{\Delta d}_{1}\right)$. This comes from the general idea which consists in using, when possible, second order approximation and activating the first order one, in critical situations. 


\section{ii) Wet/dry fronts}

All the previous descriptions need to be adapted in the presence of wet/dry fronts. We will now describe this adaptation inspired by the work [11] and here extended to the situation where the material can be fluid or plastic.

From the numerical point of view, we said that $H_{i}$ is null when $H_{i}<H_{\varepsilon}$. For the numerical tests, we set $H_{\varepsilon}=$ $5 \cdot 10^{-3}$.

In some of the cases described below, we impose no numerical diffusion in the discretization of the equation in $H$ and a local equilibrium of the pressure term. In practice, this corresponds to set the following definitions of $\Delta\left(\zeta+\delta^{n} \partial_{x} V\right)_{i+1 / 2}^{k}$ and $\bar{\sigma}_{i+1 / 2}^{k}$ :

- If $H_{i-1} \leq H_{\varepsilon}, H_{i} \leq H_{\varepsilon}, H_{i+1} \leq H_{\varepsilon}$ or $H_{i+2} \leq H_{\varepsilon}$ and the material is rigid enough in the following sense:

- for the AL algorithm, if $\left|\mu_{i+1 / 2}^{n}+r \frac{v_{i+1}-v_{i}}{\Delta x}\right|<\tau_{y} \sqrt{2}$;

- for the BM algorithm, if $\left|\theta_{i+1 / 2}^{n}+\frac{1}{\lambda_{i+1 / 2}^{n}} \frac{v_{i+1}-v_{i}}{\Delta x}\right|<H_{i+1 / 2}^{n} \tau_{y} \sqrt{2}$;

then we set the following definitions of $\Delta\left(\zeta+\delta^{n} \partial_{x} V\right)_{i+1 / 2}^{k}$ and $\bar{\sigma}_{i+1 / 2}^{k}$ :

$$
\begin{aligned}
& \Delta\left(\zeta+\delta^{n} \partial_{x} V\right)_{i+1 / 2}^{k}=-f_{z} H_{i+1 / 2}^{n}\left(b_{i+1}-b_{i}-H_{i}+\frac{f_{\Omega}}{f_{z}} \Delta x\right), \\
& \bar{\sigma}_{i+1 / 2}^{k}=\left(\begin{array}{c}
f_{\Omega} x_{i}+f_{z} b_{i}+\frac{1}{2} f_{z} H_{i} \\
\zeta_{i-1 / 2}^{k}
\end{array}\right) .
\end{aligned}
$$

- If $H_{i} \leq H_{\varepsilon}$ or $H_{i+1} \leq H_{\varepsilon}$ and if

- for the AL algorithm, if $\left|\mu_{i+1 / 2}^{n}+r \frac{v_{i+1}-v_{i}}{\Delta x}\right| \geq \tau_{y} \sqrt{2}$;

- for the BM algorithm, if $\left|\theta_{i+1 / 2}^{n}+\frac{1}{\lambda_{i+1 / 2}^{n}} \frac{v_{i+1}-v_{i}}{\Delta x}\right| \geq H_{i+1 / 2}^{n} \tau_{y} \sqrt{2}$;

we proceed as follows. Let us suppose that $H_{i}>H_{\varepsilon}$ and $H_{i+1} \leq H_{\varepsilon}$. Then, if

$$
b_{i}+H_{i}<b_{i+1}
$$

we set the definition (35). Moreover, if $V_{i+1}<0$, then we set $V_{i+1}=0$ in the computation of the numerical flux for the evolution of the height of the material. If $H_{i} \leq H_{\varepsilon}, H_{i+1}>H_{\varepsilon}$, then we apply the same treatment symmetrically.

Let us remark that in this approach, we test whether the material is fluid or rigid. For example, let us consider the case $\tau_{y}=0$, i.e. the fluid regime. In this case, it is important to check the relative position of the free surface at $x=x_{i}$ and $x=x_{i+1}$, which coincides with the wet/dry numerical treatment proposed in [11] for the shallow water equations. On the contrary, when the material is rigid enough, it is not important to check the relative position of the free surface. Because in this case, the rigidity naturally implies that the solution is at rest independently of the relative position of the free surface.

\subsection{The global coupled scheme}

In this section, we present the global scheme obtained by gathering the aforementioned discretization procedures. It allows to solve the evolution problem (13)-(14). For sake of brevity, we detail here the scheme in the case of the $\mathrm{BM}$ algorithm. For completeness, the case of the AL is completely described in Appendix D. Of note, from the implementation viewpoint, both methods share a lot in common and these similarities are embedded in a general framework (see (39)-(43) in the following).

\section{Global numerical scheme for (13)-(14) - Bermúdez-Moreno method}


- Initialization at time $t=0$ for $n=0: V^{n}, H^{n}, \theta^{n}$ are given by the initial conditions.

- Time loop: For $n=0, \ldots, n_{\max }$.

- Resolution of the problem on $V^{k+1}$

$\left\{V_{i}^{n}\right\}_{i},\left\{H_{i}^{n}\right\}_{i}$ and $\left\{\theta_{i+1 / 2}^{n}\right\}_{i}$ are known.

Compute quantities which are invariant in the following loop:

\section{Bermúdez-Moreno loop:}

[Step 0] Initialize for $k=0$ : for all $i, V_{i}^{k}=V_{i}^{n}$ and $\theta_{i+1 / 2}^{k}=\theta_{i+1 / 2}^{n}$.

[Step 1] Update $\left\{V_{i}^{k+1}\right\}_{i}$ by solving the linear system defined by the second component of (29). See details in (39)-(43), with $\delta_{i+1 / 2}^{n}$ and $\zeta_{i+1 / 2}^{k}$ given by (28)-(b) and (27)-(b).

[Step 2] Compute the auxiliary variable $\left\{\xi_{i+1 / 2}^{k+1}\right\}_{i}$ :

$$
\xi_{i+1 / 2}^{k+1}=\frac{V_{i+1}^{k}-V_{i}^{k}}{\Delta x}+\lambda \theta_{i+1 / 2}^{k}
$$

[Step 3] Update $\left\{\theta_{i+1 / 2}^{k+1}\right\}_{i}$ via

$$
\theta_{i+1 / 2}^{k+1}= \begin{cases}\frac{-\omega \xi_{i+1 / 2}^{k+1}+\tau_{y} \sqrt{2} H_{i+1 / 2}^{n}}{1-\lambda \omega} & \text { if } \xi_{i+1 / 2}^{k+1}>\lambda \tau_{y} \sqrt{2} H_{i+1 / 2}^{n}, \\ \frac{\xi_{i+1 / 2}^{k+1}}{\lambda} & \text { if } \xi_{i+1 / 2}^{k+1} \in\left[-\lambda \tau_{y} \sqrt{2} H_{i+1 / 2}^{n}, \lambda \tau_{y} \sqrt{2} H_{i+1 / 2}^{n}\right], \\ \frac{-\omega \xi_{i+1 / 2}^{k+1}-\tau_{y} \sqrt{2} H_{i+1 / 2}^{n}}{1-\lambda \omega} & \text { if } \xi_{i+1 / 2}^{k+1}<-\lambda \tau_{y} \sqrt{2} H_{i+1 / 2}^{n} .\end{cases}
$$

[Step 4] Set: for all $i, V_{i}^{k}=V_{i}^{k+1}, \theta_{i+1 / 2}^{k}=\theta_{i+1 / 2}^{k+1}$ and return to Step 1.

[Step 5] At convergence, when condition (20) is verified, set $V_{i}^{n+1}=V_{i}^{k+1}$ and $\theta_{i+1 / 2}^{n+1}=\theta_{i+1 / 2}^{k+1} \forall i$.

- Resolution of the problem on $H^{k+1}$

Compute $H^{n+1}=H^{k+1}$ with the finite volume method defined by the first component of (29), defined in terms of the most recent multiplier $\left\{\theta_{i+1 / 2}^{n+1}\right\}_{i}$ and taking into account the wet/dry treatment presented before where needed.

It is worth giving some more details about [Step 1]. Remark that the second component of (29) defines a linear system where the unknowns are $\left\{V_{i}^{k+1}\right\}_{i}$. If we denote $V^{k+1}$ the vector whose $i^{\text {th }}$ component is $V_{i}^{k+1}$, the aforementioned linear system can be written as

$$
\boldsymbol{A}^{n} V^{k+1}=\boldsymbol{b}^{n, k},
$$

where $\boldsymbol{A}^{n}$ is a matrix defined in terms of $\left\{H_{i}^{n}\right\}_{i}$; consequently, $\boldsymbol{A}^{n}$ does not change during the duality loop (in $k$ ). As a matter of fact, $\boldsymbol{A}^{n}$ is a tridiagonal matrix, whose line $i$ is defined by the following entries:

$$
\begin{gathered}
\boldsymbol{A}_{i, i-1}^{n}=\frac{-4 \eta}{\Delta x^{2}} H_{i-1 / 2}^{n}-\frac{\delta_{i-1 / 2}^{n}}{\Delta x^{2}}, \quad \boldsymbol{A}_{i, i+1}^{n}=\frac{-4 \eta}{\Delta x^{2}} H_{i+1 / 2}^{n}-\frac{\delta_{i+1 / 2}^{n}}{\Delta x^{2}}, \\
\boldsymbol{A}_{i, i}^{n}=\frac{H_{i}^{n}}{\Delta t}+\frac{4 \eta}{\Delta x^{2}}\left(H_{i-1 / 2}^{n}+H_{i+1 / 2}^{n}\right)+\frac{1}{\Delta x^{2}}\left(\delta_{i-1 / 2}^{n}+\delta_{i+1 / 2}^{n}\right)+\beta .
\end{gathered}
$$

On the contrary, the right hand side of the linear system (39) changes for each iteration in $k$. The $i^{\text {th }}$ component of $\boldsymbol{b}^{n, k}$ is decomposed as

$$
\boldsymbol{b}_{i}^{n, k}=\boldsymbol{b}_{i}^{n,(1)}+\boldsymbol{b}_{i}^{n, k,(2)}+\boldsymbol{b}_{i}^{n, k,(3)},
$$


where

$$
\begin{aligned}
\boldsymbol{b}_{i}^{n,(1)} & =H_{i}^{n}\left(f_{\Omega}+f_{z} \frac{b_{i+1}-b_{i-1}}{2 \Delta x}\right), \\
\boldsymbol{b}_{i}^{n, k,(2)} & =\frac{\zeta_{i+1 / 2}^{k}-\zeta_{i-1 / 2}^{k}}{\Delta x}, \\
\boldsymbol{b}_{i}^{n, k,(3)} & =H_{i}^{n} \frac{\left[\phi\left(W_{i-1}^{n}, W_{i}^{n},\left\{\zeta_{j+1 / 2}^{k}\right\}_{j=i-1}^{j=i+1}\right)\right]_{2}-\left[\phi\left(W_{i}^{n}, W_{i+1}^{n},\left\{\zeta_{j+1 / 2}^{k}\right\}_{j=i-1}^{j=i+1}\right)\right]_{2}}{\Delta x} .
\end{aligned}
$$

Note that all of this is applied only where $\frac{1}{2}\left(H_{i-1 / 2}^{n}+H_{i+1 / 2}^{n}\right) \geq H_{\varepsilon}$. Indeed, on the contrary, we set $\boldsymbol{A}_{i, i-1}^{n}=\boldsymbol{A}_{i, i+1}^{n}=$ $0, \boldsymbol{A}_{i, i}^{n}=1, \boldsymbol{b}_{i}^{n, k}=0$.

\subsection{Well-balanced properties}

In this section, we study the well-balanced properties of the proposed numerical scheme. Concretely, we are interested in studying stationary solutions defined by a horizontal free surface or by a free surface parallel to the reference slope with variable bottom, for rigid enough materials (see Figure 2).

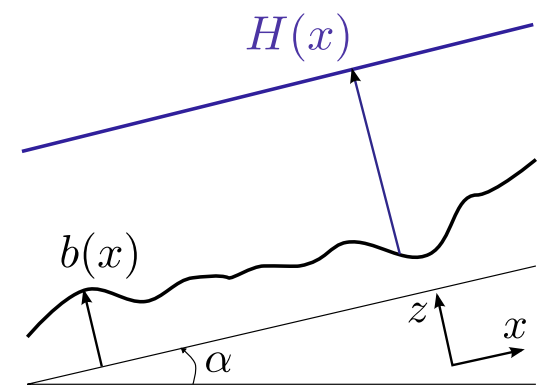

Figure 2: Stationary solution with free surface parallel to the reference plane.

The first type of stationary solution corresponds to a material at rest with zero velocity and a horizontal free surface. It is a stationary solution of the system independently of the rigidity of the material. For the second type, we consider a stationary solution with zero velocity and verifying $b(x)+H(x)=C, \forall x \in[0, L]$, where $C$ is a constant value. Let us study the conditions on such a solution. One should have

$$
\int_{0}^{L} \tau_{y} \sqrt{2} H\left|\partial_{x} \psi\right| d x \geq \int_{0}^{L}\left(H\left(f_{\Omega}+f_{z} \partial_{x} b\right) \psi-f_{z} \frac{H^{2}}{2} \partial_{x} \psi\right) d x, \quad \forall \psi .
$$

Taking into account that $\partial_{x} b=-\partial_{x} H$ and integrating by parts, this condition is equivalent to

$$
\int_{0}^{L} \tau_{y} \sqrt{2} H\left|\partial_{x} \psi\right| d x \geq \int_{0}^{L} H f_{\Omega} \psi d x=\int_{0}^{L}\left(-f_{\Omega} \int_{0}^{x} H(s) d s+c\right) \partial_{x} \psi d x
$$

We can set $c=f_{\Omega} \int_{0}^{L / 2} H(x) d x$. Then, this condition is verified if

$$
\left|f_{\Omega} \int_{L / 2}^{x} H(s) d s\right| \leq \tau_{y} \sqrt{2} H(x) \quad \forall x \in[0, L] .
$$

We can also obtain the analytic value to which (i) $\mu$ converges in the case of the Augmented Lagrangian method, and (ii) $\theta$ for the Bermúdez-Moreno method. 
For the AL or the BM algorithms, in the case of a stationary solution with zero velocity, we have the following equation:

$$
\partial_{x}\left(\frac{f_{\Omega}}{2} H^{2}\right)=H\left(f_{\Omega}+f_{z} \partial_{x} b\right)+\partial_{x} \zeta
$$

with

$$
\zeta(x)= \begin{cases}H(x) \mu(x) & \text { in the case of AL, } \\ \theta(x) & \text { in the case of BM. }\end{cases}
$$

Taking into account that the stationary solution that we consider in this test verifies $b+H=C$, this equation simplifies to the following one:

$$
-H f_{\Omega}=\partial_{x}(\zeta)
$$

Then,

$$
\zeta=-f_{\Omega} \int_{L / 2}^{x} H(x) d x
$$

We have the following result for the proposed algorithms:

Theorem 1. The AL and the BM algorithms preserve exactly the following two types of stationary solutions:

i) Material at rest with free surface parallel to the reference slope:

$$
V=0, \quad b+H=\text { constant }
$$

for any given bottom function $b(x)$, if the material is rigid enough, i.e. if $\tau_{y}$ is such that the following discrete version of (44) holds:

$$
\left|f_{\Omega} \Delta x\left(\sum_{j=1}^{i} H_{j}^{0}-\sum_{j=1}^{[N / 2]} H_{j}^{0}\right)\right| \leq \tau_{y} \sqrt{2} H_{i+1 / 2}^{0} \quad \forall i .
$$

ii) Material at rest with horizontal free surface:

$$
V=0, \quad f_{\Omega} x+f_{z}(H+b)=\text { constant },
$$

if $\left\{\zeta_{i+1 / 2}\right\}_{i=1}^{N}$ is initialized as follows:

$$
\zeta_{i+1 / 2}^{0}=-\Delta x\left(\sum_{j=1}^{i} H_{j}^{0}-\sum_{j=1}^{[N / 2]} H_{j}^{0}\right)\left(f_{\Omega}+f_{z} \frac{H_{i+1}^{0}+b_{i+1}-\left(H_{i}^{0}+b_{i}\right)}{\Delta x}\right),
$$

where [N/2] is the integer part of $N / 2$, being $N$ the number of points of the space mesh.

The proof is given in Appendix E.

\section{Numerical comparison of both approaches}

In this section, we present numerical tests to illustrate the good properties of the schemes presented above.

A first natural test is to check the order of convergence in space on a non-trivial stationary problem, in order to test the accuracy of the duality methods for variational inequalities (thus in absence of well-balancing and wet/dry front issues). It appears that the viscoplastic model (16)-(17) degenerates to the well-known Poiseuille-Bingham flow for which an analytic solution is known. All the details of this test are given in Appendix F. The conclusions are that both the Augmented Lagrangian and the Bermúdez-Moreno methods are at least of order two (in $L^{2}$-norm) in space for the velocity of this non-zero stationary solution. In terms of computational cost, for this specific test and a fixed duality parameter (either $r$ or $\omega$ ), it is shown that the Bermúdez-Moreno is approximately $20 \%$ cheaper than the Augmented Lagrangian .

We then focus on the main novelty of this paper, i.e. the well-balanced properties of the schemes. 


\subsection{Well-balanced tests}

\section{Test 5.1.a: Analytical stationary solution}

In this test we study the error and convergence of the numerical results for the stationary solution studied in Section 4.2. Concretely, we consider a domain of length $L=10$ and a solution defined by

$$
V=0, \quad H(x)=2-b(x), \quad b(x)=\cos (\pi x),
$$

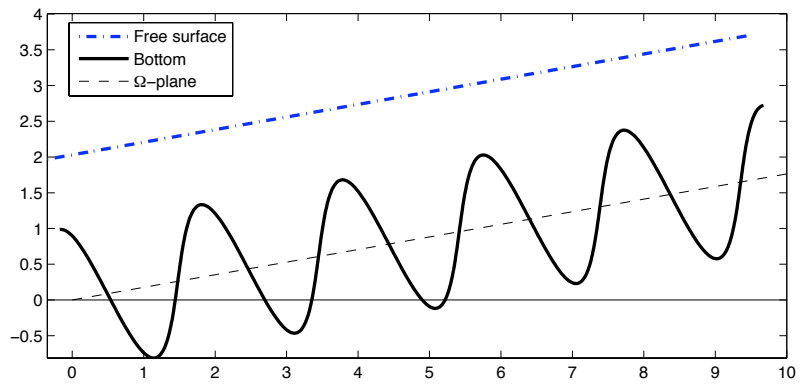

Figure 3: Test 5.1.a. Free surface, bottom and $\Omega$-plane.

as shown in Figure 3. This is a stationary solution of the system if the material is rigid enough. For this definition of $H(x)$ we can compute condition (44) exactly; we obtain that it is a stationary solution of the system if $\tau_{y}$ verifies

$$
\tau_{y} \geq \max _{x \in[0,10]} \frac{\left|g \sin \alpha\left(2(5-x)-\frac{\sin (\pi x)}{\pi}\right)\right|}{\sqrt{2}(2-\cos (\pi x))} .
$$

For this test we set $\alpha=10^{\circ}$, so $\tau_{y}$ must be approximately greater than 17.03.

We compare the numerical results with the analytical ones corresponding to $H(x)$ and the multipliers $\mu$ and $\theta$. By (45) and (46) we have that

$$
\mu(x)=\frac{2(x-5)-\sin (\pi x) / \pi}{2-\cos (\pi x)} g \sin \alpha, \quad \theta(x)=\left(2(x-5)-\frac{\sin (\pi x)}{\pi}\right) g \sin \alpha .
$$

Following Theorem 1, if we initialize the multipliers with (48) then the stationary solution is exactly preserved, up to machine precision. Then, we initialize both multipliers to zero in order to study their convergence to the analytical values.

In Figure 4, we present the convergence of $\mu$ and $\theta$ to the analytical solution when both quantities are initialized to zero, for 100 computational cells. In Figure 5, we study the numerical order of convergence in space, through a mesh refinement. For $\mu$ and $\theta$ first order is reached by computing the error in the $L^{\infty}$ norm, and second order for the $L^{2}$ error. For the BM algorithm the parameter $\omega$ was set to $\omega_{\text {opt }}$ (equation (22)), while for the AL method we have set $r=10$.

In Figure 6, we present a comparison of the number of iterations $k_{\text {end }}$ necessary to converge in the iterative algorithm of the Augmented Lagrangian and the Bermúdez-Moreno methods, for the first iteration in time. We also picture a vertical line corresponding to the optimal parameter for the BM method.

For the BM method we have proposed two different ways to define the parameter $\omega$, as a constant value in space (but variable in time), or depending on the thickness of the material layer: $\omega=H^{n}(x) \varpi$, being $\varpi$ a constant value in space. Then, we denote by $\operatorname{BM}(\omega)$ the results corresponding to the choice of $\omega$ as a constant parameter, and by $\operatorname{BM}(\varpi)$ the results corresponding to $\omega=H^{n}(x) \varpi$.

For $\operatorname{BM}(\omega)$, the optimal parameter $w_{\text {opt }}$ is defined by (22), equals to 20.5 approximately for this test. While for $\mathrm{BM}(\varpi)$ the optimal parameter $\varpi_{\mathrm{opt}}$ is defined by (23), equals to 10.3 approximately for this test. Note that both, $\omega_{\mathrm{opt}}$ and $\varpi_{\mathrm{opt}}$ are near to the optimal value obtained numerically, which are respectively 26 and 21. 


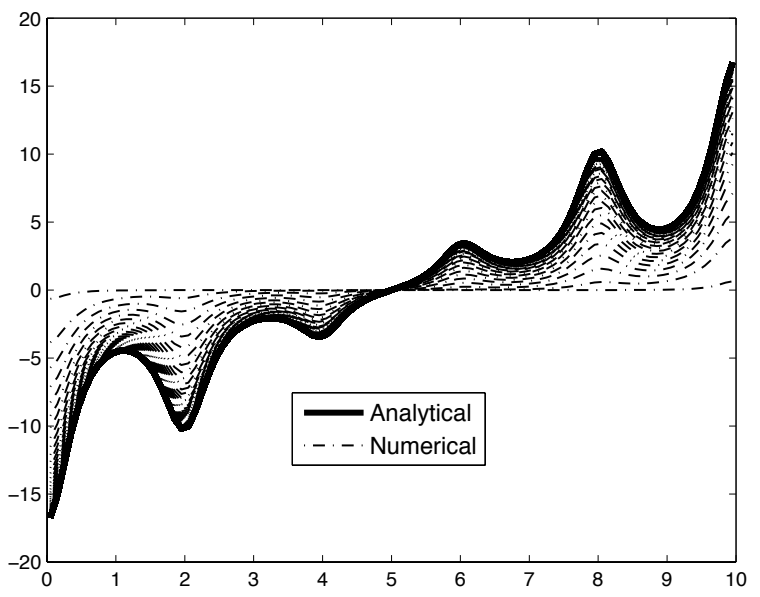

(a) $\mu(x)$

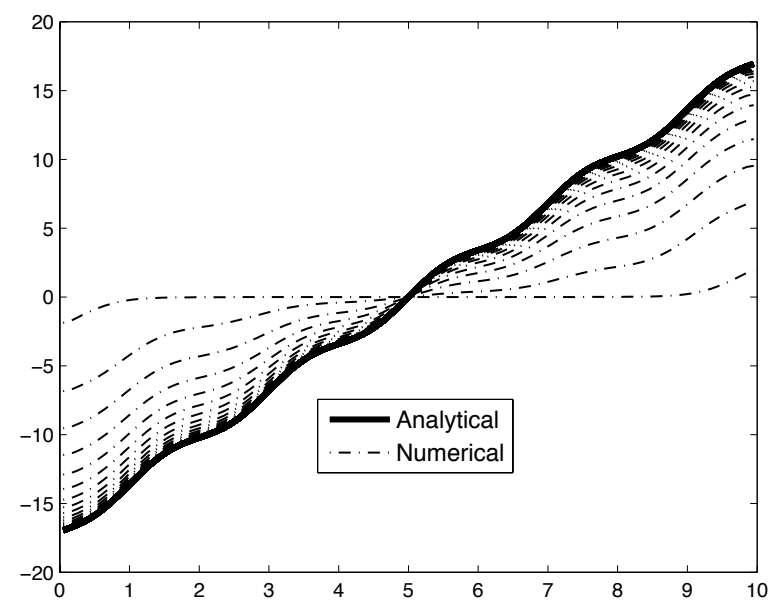

(b) $\theta(x)$

Figure 4: Test 5.1.a. Convergence of $\mu$ and $\theta$ to the analytical values

The results presented in Figure 6 correspond to $\Delta x=0.1$ and $\{\omega, r, \varpi\} \in[1,300]$, concretely a partition with subintervals of length equals to 5. Let us remark that for $\omega=r=\varpi=1$ the $\operatorname{BM}(\varpi)$ is the one that need a smaller number of iterations, after the $\operatorname{BM}(\omega)$ and the one that needs a greater number of iterations is the AL. This behavior is also observed for the values of the parameters smaller than the optimal one. When they are greater than the optimal parameter both versions of the BM algorithm present a similar number of iterations. Nevertheless, for the Augmented Lagrangian method we observe that the number of iterations decreases when the value of $r$ increases. This is a phenomena that has been yet observed in the case of Augmented Lagrangian method for other applications. Nevertheless, from a practical point of view, the choice of bigger values of $r$, when applied to other numerical tests, can imply some problems of stability and ill-conditioning problems in the linear system related to the iterative algorithm (see [19]).

Finally, we study the influence of the angle on the numerical solution. Again we initialize the multipliers to zero and we compare the errors for $V(x)$ and $H(x)$ corresponding to $\alpha=10^{\circ}$ and $\alpha=45^{\circ}$ through a mesh refinement, see Tables 1 and 2. We observe that errors are almost independent of the angle and the grid.

\section{Test 5.1.b: Stationary solution on a random bottom}

In this test, we consider a random bottom and wet/dry fronts. We also consider two initial conditions to set the position of the free surface: horizontal or parallel to $\tan (\alpha) x$ (see Figure 7). Concretely, we set the following two definitions of the height of the material layer:

$$
H(x)=\max \left(z_{r e f, 1}-b(x), 0\right), \quad \text { or } \quad H(x)=\max \left(\frac{z_{\text {ref }, 2}}{\cos (\alpha)}-b(x)-\tan (\alpha) x, 0\right) .
$$

where the bottom function has been defined as $b(x)=r_{1}(x)\left(1+r_{2}(x)\right) e^{r_{3}(x)}$, where $r_{j}(x) \in[0,1], j=1,2,3$, are three random numbers for each value of $x$ (see Figure 7). $z_{\text {ref, } 1}$ corresponds to the height of the material on the $\Omega$-plane, while $z_{r e f, 2}$ corresponds to the level of the horizontal free surface. For example, Figure 7 is obtained with $z_{r e f, 1}=2$, $z_{r e f, 2}=3$. With the purpose to consider the case with and without wet/dry fronts, we set two different values of $z_{\text {ref }, 1}$ and $z_{\text {ref }, 2}$, concretely:

$$
z_{\text {ref }, 1}=2, z_{r e f, 1}=5 \quad \text { and } \quad z_{r e f, 2}=3, z_{r e f, 2}=5.8 .
$$

The numerical results presented in this test correspond to $\alpha=10^{\circ}$. Analogously to the previous tests, we obtain similar results if we increase the angle. For this test we set $\Delta x=0.1$.

The multipliers $\mu$ and $\theta$ are initialized to zero. In Figure 8 we present the convergence of $\mu$ and $\theta$ to (48). This theoretical value has been used in Theorem 1 to prove that, with this initialization of the multipliers, the proposed 


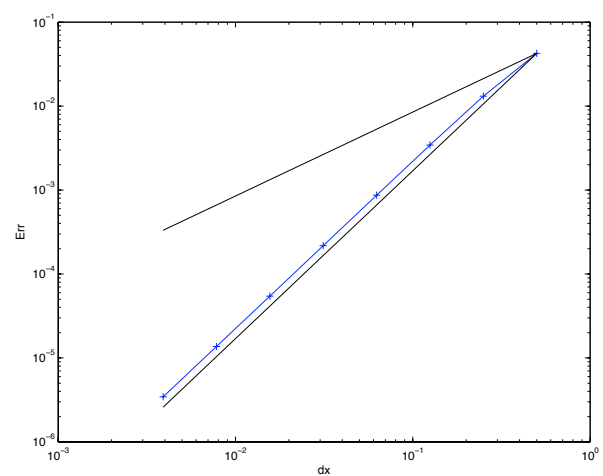

(a) BM Convergence on $\theta \cdot \mathrm{L}^{\infty}$ error.

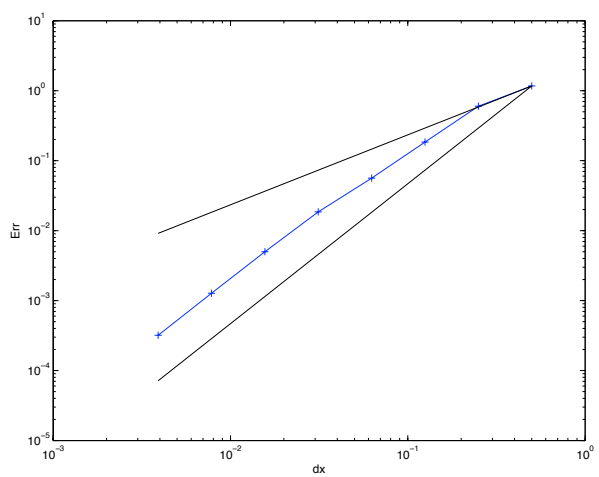

(c) AL Convergence on $\mu$. $\mathrm{L}^{\infty}$ error.

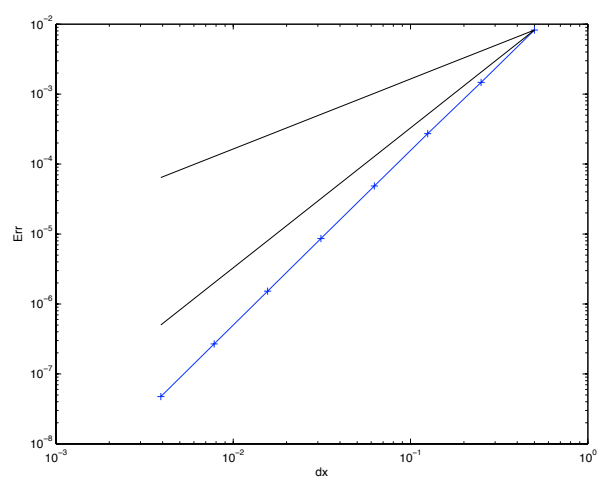

(b) BM Convergence on $\theta . \mathrm{L}^{2}$ error.

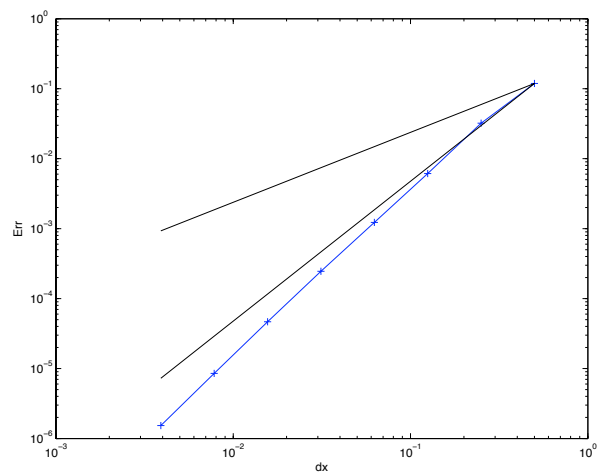

(d) AL Convergence on $\mu . \mathrm{L}^{2}$ error.

Figure 5: Test 5.1.a. Order of convergence of $\theta$ and $\mu$ for the BM and $\mathrm{AL}$ algorithms. Black continuous lines show first and second order of convergence; blue line with + is the computed error.

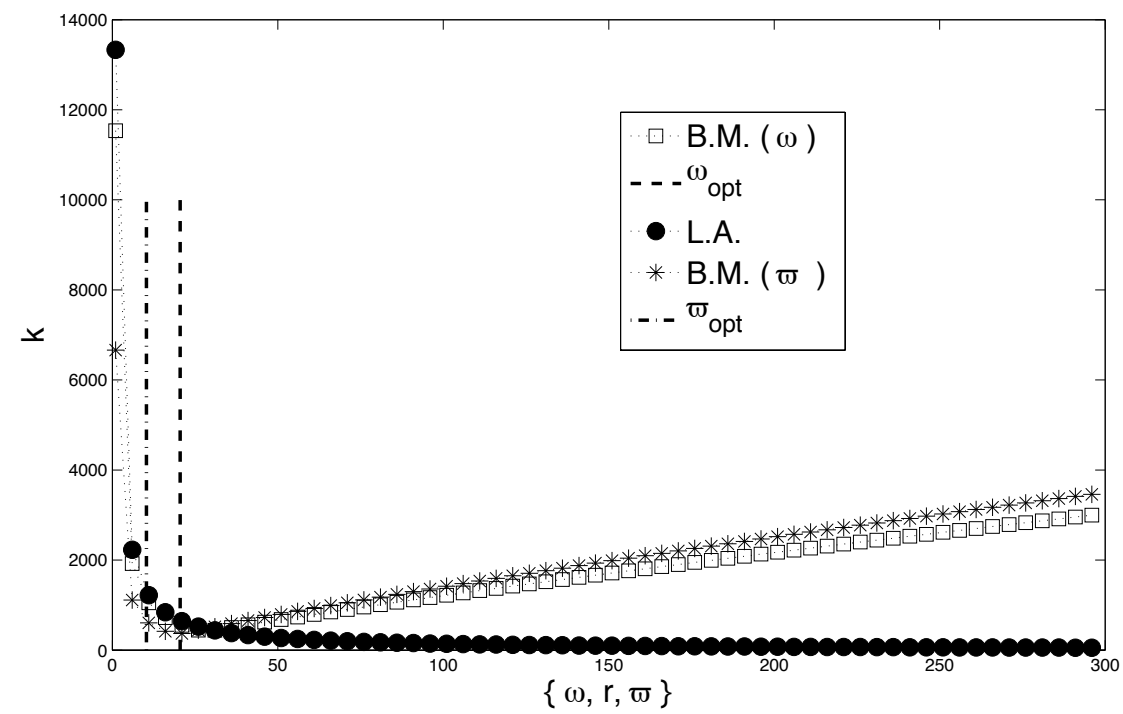

Figure 6: Test 5.1.a. Number of iterations in terms of $r$ (for AL), $\omega$ and $\varpi$ (for BM). 
Table 1: Test 5.1.a. Errors for $V(x)$ and $H(x)$ for BM.

\begin{tabular}{c|c|cc|cc}
\hline$\alpha$ & Cells & $\mathrm{V} L^{\infty}$ Error & $\mathrm{V} L^{2}$ Error & $\mathrm{H} L^{\infty}$ Error & $\mathrm{H} L^{2}$ Error \\
\hline $10^{\circ}$ & 20 & $2.703 \mathrm{E}-09$ & $4.271 \mathrm{E}-10$ & $6.471 \mathrm{E}-10$ & $8.643 \mathrm{E}-11$ \\
& 40 & $1.948 \mathrm{E}-09$ & $2.196 \mathrm{E}-10$ & $5.950 \mathrm{E}-10$ & $5.082 \mathrm{E}-11$ \\
& 80 & $9.234 \mathrm{E}-10$ & $7.323 \mathrm{E}-11$ & $4.465 \mathrm{E}-10$ & $2.588 \mathrm{E}-11$ \\
& 160 & $1.704 \mathrm{E}-10$ & $9.552 \mathrm{E}-12$ & $2.482 \mathrm{E}-10$ & $1.008 \mathrm{E}-11$ \\
& 320 & $3.103 \mathrm{E}-10$ & $1.222 \mathrm{E}-11$ & $1.254 \mathrm{E}-10$ & $3.591 \mathrm{E}-12$ \\
& 640 & $5.229 \mathrm{E}-10$ & $1.456 \mathrm{E}-11$ & $1.857 \mathrm{E}-11$ & $3.740 \mathrm{E}-13$ \\
& 1280 & $1.974 \mathrm{E}-10$ & $3.884 \mathrm{E}-12$ & $2.168 \mathrm{E}-11$ & $3.106 \mathrm{E}-13$ \\
& 2560 & $2.449 \mathrm{E}-10$ & $3.406 \mathrm{E}-12$ & $4.742 \mathrm{E}-12$ & $4.786 \mathrm{E}-14$ \\
\hline $45^{\circ}$ & 20 & $2.917 \mathrm{E}-09$ & $4.554 \mathrm{E}-10$ & $7.021 \mathrm{E}-10$ & $9.259 \mathrm{E}-11$ \\
& 40 & $1.898 \mathrm{E}-09$ & $2.131 \mathrm{E}-10$ & $6.851 \mathrm{E}-10$ & $5.823 \mathrm{E}-11$ \\
& 80 & $1.175 \mathrm{E}-09$ & $9.311 \mathrm{E}-11$ & $4.553 \mathrm{E}-10$ & $2.637 \mathrm{E}-11$ \\
& 160 & $4.501 \mathrm{E}-10$ & $2.516 \mathrm{E}-11$ & $3.043 \mathrm{E}-10$ & $1.236 \mathrm{E}-11$ \\
& 320 & $9.862 \mathrm{E}-11$ & $3.877 \mathrm{E}-12$ & $1.567 \mathrm{E}-10$ & $4.487 \mathrm{E}-12$ \\
& 640 & $3.981 \mathrm{E}-10$ & $1.108 \mathrm{E}-11$ & $5.827 \mathrm{E}-11$ & $1.178 \mathrm{E}-12$ \\
& 1280 & $3.564 \mathrm{E}-10$ & $7.011 \mathrm{E}-12$ & $8.732 \mathrm{E}-12$ & $1.256 \mathrm{E}-13$ \\
& 2560 & $6.904 \mathrm{E}-11$ & $9.600 \mathrm{E}-13$ & $1.571 \mathrm{E}-11$ & $1.589 \mathrm{E}-13$ \\
\hline
\end{tabular}

Table 2: Test 5.1.a. Errors for $V(x)$ and $H(x)$ for $\operatorname{AL}(r=10)$.

\begin{tabular}{c|c|cc|cc}
\hline$\alpha$ & Cells & $\mathrm{V} L^{\infty}$ Error & $\mathrm{V} L^{2}$ Error & $\mathrm{H} L^{\infty}$ Error & $\mathrm{H} L^{2}$ Error \\
\hline $10^{\circ}$ & 20 & $3.593 \mathrm{E}-10$ & $5.038 \mathrm{E}-11$ & $3.300 \mathrm{E}-09$ & $4.946 \mathrm{E}-10$ \\
& 40 & $4.887 \mathrm{E}-10$ & $4.150 \mathrm{E}-11$ & $2.184 \mathrm{E}-09$ & $2.396 \mathrm{E}-10$ \\
& 80 & $4.106 \mathrm{E}-10$ & $2.401 \mathrm{E}-11$ & $1.268 \mathrm{E}-09$ & $9.998 \mathrm{E}-11$ \\
& 160 & $2.997 \mathrm{E}-10$ & $1.218 \mathrm{E}-11$ & $7.103 \mathrm{E}-10$ & $3.964 \mathrm{E}-11$ \\
& 320 & $1.813 \mathrm{E}-10$ & $5.172 \mathrm{E}-12$ & $1.792 \mathrm{E}-10$ & $7.054 \mathrm{E}-12$ \\
& 640 & $8.896 \mathrm{E}-11$ & $1.789 \mathrm{E}-12$ & $1.815 \mathrm{E}-10$ & $5.037 \mathrm{E}-12$ \\
& 1280 & $2.219 \mathrm{E}-11$ & $3.147 \mathrm{E}-13$ & $3.449 \mathrm{E}-10$ & $6.764 \mathrm{E}-12$ \\
& 2560 & $1.273 \mathrm{E}-11$ & $1.279 \mathrm{E}-13$ & $2.105 \mathrm{E}-10$ & $2.919 \mathrm{E}-12$ \\
\hline $45^{\circ}$ & 20 & $3.226 \mathrm{E}-10$ & $4.666 \mathrm{E}-11$ & $3.328 \mathrm{E}-09$ & $4.975 \mathrm{E}-10$ \\
& 40 & $4.637 \mathrm{E}-10$ & $3.997 \mathrm{E}-11$ & $2.148 \mathrm{E}-09$ & $2.352 \mathrm{E}-10$ \\
& 80 & $4.064 \mathrm{E}-10$ & $2.371 \mathrm{E}-11$ & $1.315 \mathrm{E}-09$ & $1.032 \mathrm{E}-10$ \\
& 160 & $3.047 \mathrm{E}-10$ & $1.238 \mathrm{E}-11$ & $7.871 \mathrm{E}-10$ & $4.389 \mathrm{E}-11$ \\
& 320 & $1.946 \mathrm{E}-10$ & $5.549 \mathrm{E}-12$ & $2.903 \mathrm{E}-10$ & $1.142 \mathrm{E}-11$ \\
& 640 & $1.038 \mathrm{E}-10$ & $2.087 \mathrm{E}-12$ & $8.149 \mathrm{E}-11$ & $2.259 \mathrm{E}-12$ \\
& 1280 & $3.619 \mathrm{E}-11$ & $5.137 \mathrm{E}-13$ & $2.924 \mathrm{E}-10$ & $5.733 \mathrm{E}-12$ \\
& 2560 & $6.308 \mathrm{E}-12$ & $6.349 \mathrm{E}-14$ & $2.538 \mathrm{E}-10$ & $3.518 \mathrm{E}-12$ \\
\hline
\end{tabular}

numerical scheme preserves exactly both types of stationary solutions. Tables 3 and 4 present the errors for $V(x)$ and $H(x)$, when $\mu$ and $\theta$ are initialized to zero. 

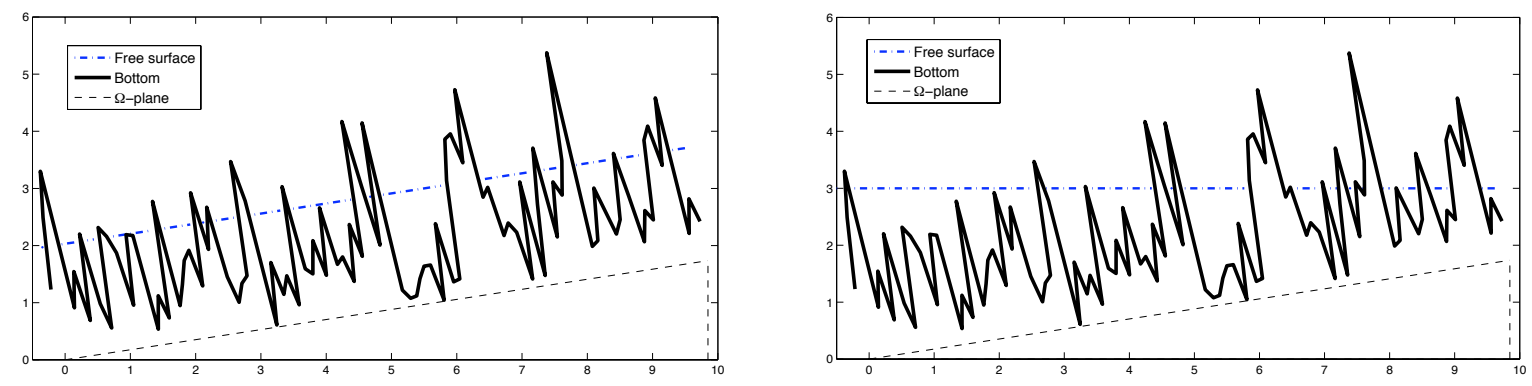

Figure 7: Test 5.1.b. Free surface, bottom and $\Omega$-plane.

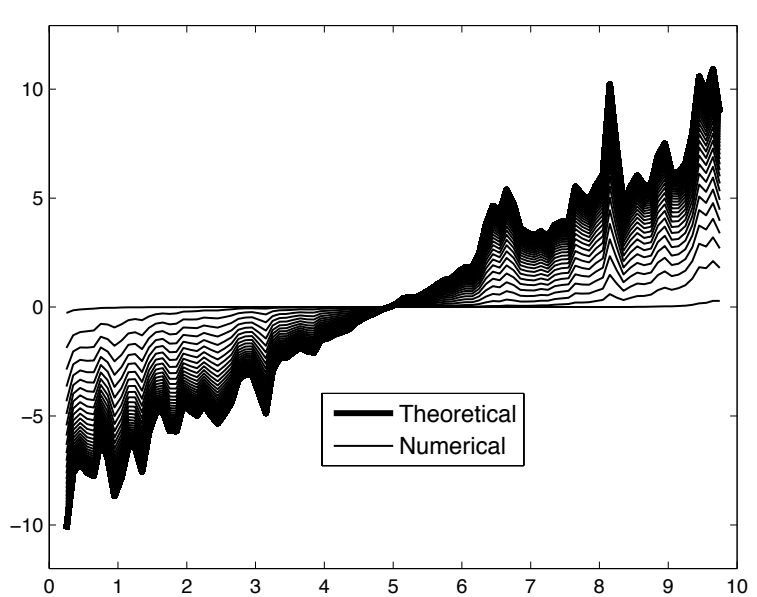

(a) $\mu(x)$

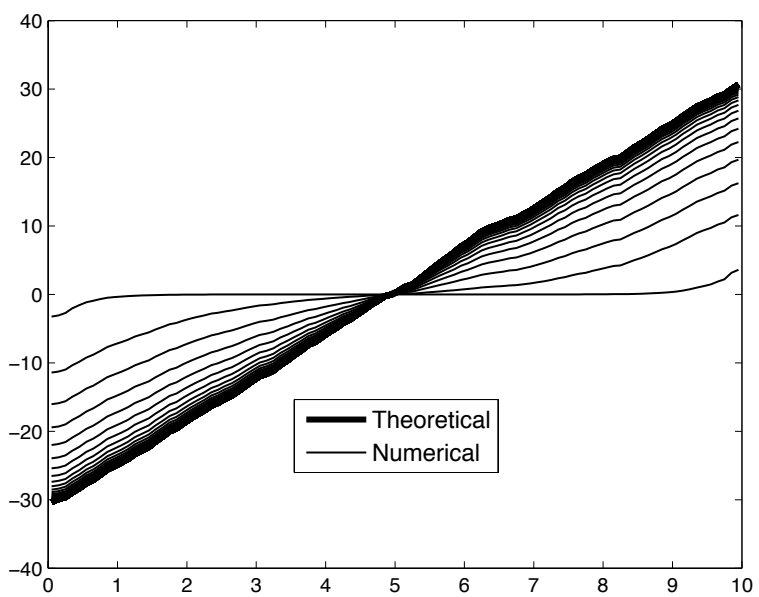

(b) $\theta(x)$

Figure 8: Test 5.1.b. Initial condition: $H(x)=\max \left(z_{r e f, 1}-b(x), 0\right)$ with $z_{r e f, 1}=5$. Convergence of $\mu$ and $\theta$ to the theoretical value (48).

Table 3: Test 5.1.b. Initial condition: $H(x)=\max \left(z_{r e f, 1}-b(x), 0\right)$. Errors for $V(x)$ and $H(x)$.

\begin{tabular}{c|c|c|cc|c}
\hline$z_{r e f, 1}$ & $\left\{\zeta_{i+1 / 2}^{1}\right\}_{i}$ & $\mathrm{~V} L^{\infty}$ Error & $\mathrm{V} L^{2}$ Error & $\mathrm{H} L^{\infty}$ Error & $\mathrm{H} L^{2}$ Error \\
\hline 2 & $\mathrm{BM}\left(\omega_{\mathrm{opt}}\right)$ & $1.164 \mathrm{E}-11$ & $1.536 \mathrm{E}-13$ & $1.257 \mathrm{E}-08$ & $1.772 \mathrm{E}-10$ \\
& $\mathrm{BM}\left(\varpi_{\mathrm{opt}}\right)$ & $3.672 \mathrm{E}-09$ & $5.301 \mathrm{E}-11$ & $1.210 \mathrm{E}-08$ & $2.780 \mathrm{E}-10$ \\
& $\mathrm{AL}(r=10)$ & $4.978 \mathrm{E}-10$ & $1.117 \mathrm{E}-11$ & $8.800 \mathrm{E}-10$ & $1.103 \mathrm{E}-11$ \\
\hline \multirow{2}{*}{5} & $\mathrm{BM}\left(\omega_{\mathrm{opt}}\right)$ & $3.109 \mathrm{E}-10$ & $2.191 \mathrm{E}-11$ & $8.731 \mathrm{E}-10$ & $2.780 \mathrm{E}-11$ \\
& $\mathrm{BM}\left(\varpi_{\mathrm{opt}}\right)$ & $3.414 \mathrm{E}-10$ & $2.415 \mathrm{E}-11$ & $8.733 \mathrm{E}-10$ & $2.811 \mathrm{E}-11$ \\
& $\mathrm{AL}(r=10)$ & $8.847 \mathrm{E}-10$ & $6.203 \mathrm{E}-11$ & $9.557 \mathrm{E}-10$ & $2.962 \mathrm{E}-11$ \\
\hline
\end{tabular}

\subsection{Avalanche with obstacle}

For this test we consider the case of an avalanche on an inclined plane with an obstacle. Concretely, we set $\alpha=30^{\circ}$ and

$$
b(x)=14 \mathrm{e}^{-x^{2} / 1.2}+2 \mathrm{e}^{-(x-5)^{4} / 0.1}+4 \mathrm{e}^{-(x-10)^{2} / 0.8} .
$$


Table 4: Test 5.1.b. Initial condition: $H(x)=\max \left(\frac{z_{r e f, 2}}{\cos (\alpha)}-b(x)-\tan (\alpha) x, 0\right)$. Errors for $V(x)$ and $H(x)$.

\begin{tabular}{c|c|cc|cc}
\hline$z_{r e f, 2}$ & Method & $\mathrm{V} L^{\infty}$ Error & $\mathrm{V} L^{2}$ Error & $\mathrm{H} L^{\infty}$ Error & $\mathrm{H} L^{2}$ Error \\
\hline 3 & $\mathrm{BM}\left(\omega_{\text {opt }}\right)$ & $6.316 \mathrm{E}-17$ & $1.078 \mathrm{E}-18$ & $1.662 \mathrm{E}-11$ & $3.325 \mathrm{E}-13$ \\
& $\mathrm{BM}\left(\varpi_{\text {opt }}\right)$ & $6.848 \mathrm{E}-17$ & $1.695 \mathrm{E}-18$ & $3.317 \mathrm{E}-10$ & $6.634 \mathrm{E}-12$ \\
& $\mathrm{AL}(r=10)$ & $3.933 \mathrm{E}-17$ & $1.430 \mathrm{E}-18$ & $2.220 \mathrm{E}-16$ & $4.680 \mathrm{E}-18$ \\
\hline \multirow{2}{*}{5.8} & $\mathrm{BM}\left(\omega_{\text {opt }}\right)$ & $1.951 \mathrm{E}-16$ & $1.094 \mathrm{E}-17$ & $1.776 \mathrm{E}-15$ & $4.721 \mathrm{E}-17$ \\
& $\mathrm{BM}\left(\varpi_{\text {opt }}\right)$ & $1.700 \mathrm{E}-16$ & $1.004 \mathrm{E}-17$ & $1.332 \mathrm{E}-15$ & $4.569 \mathrm{E}-17$ \\
& $\mathrm{AL}(r=10)$ & $7.430 \mathrm{E}-17$ & $3.760 \mathrm{E}-18$ & $8.882 \mathrm{E}-16$ & $3.320 \mathrm{E}-17$ \\
\hline
\end{tabular}

As initial condition, we set $V=0$ and (see Figure 9),

$$
H(x)= \begin{cases}4-b(x) & \text { if } x \in[7,9] \\ 0 & \text { otherwise. }\end{cases}
$$

We study the influence of the rigidity coefficient on the evolution of the avalanche and the final solution at rest. The length of the domain is $L=10$.

In Figures 10, 11, 12 the evolution of the avalanche for $\tau_{y} \in\{1,4,8,12\}$ is presented at times $t=1, t=1.5$, and $t=2$. In Figure 13, we plot the stationary solution reached for each value of $\tau_{y}$. In these figures, we only present the results obtained with the Bermúdez-Moreno method. Of note, the results are exactly the same with Augmented Lagrangian method, so we do not present them for sake of brevity. We have considered 200 computational cells. On the left column of these figures, we present the evolution of the free surface. Right column corresponds to the velocity. We can remark zones with evidence of rigidity: we can distinguish clearly some zones with constant velocity, that is, zones where the material moves as a block. We can remark two difficulties of this test related with the wet/dry front. First, in the evolution of the avalanche, the obstacle in the middle of the domain splits the avalanche in two parts. Second, the part of the avalanche arriving at the far left of the domain goes up on a high bed which limits its movement. This leads to a back and forth motion that eventually ends to a stationary state when all the material becomes rigid. This back and forth motion goes faster to stationary state when $\tau_{y}$ increases. But the associated free surface has a more complex shape, which is also due to the complex, non-linear, interaction of the material when it passes over the obstacle inducing the splitting of the material in the two basins.

For $\tau_{y}=1$ we can observe in Figure 13(a) that the stationary solution is close to the one of a fluid, that is, a horizontal free surface. The bump in the middle of the domain produces that the solution is divided in two parts and two different levels of the free surface. In Figures 13(c)-13(g) we can observe the influence of the rigidity of the material on the final stationary solution. Let us also remark that the computed velocity at the stationary solution are in all cases of order $10^{-9}$.

In Figure 14 a comparison of the free surface at rest with a mesh refinement is presented. The results correspond to 200, 400, 800 and 1600 computational cells. For all cases of $\tau_{y}$ we can remark that the position and form of both parts in which is divided the avalanche by the obstacle in the middle of the domain agree with the mesh refinement. When $\tau_{y}$ increases we can observe that it is more difficult to capture exactly the shape of the free surface, although the averaged form is well captured in any case.

In Figure 15 we present the computational cost until $t=1$ (sum of the number of iterations in the duality loops at each time step) with respect to the duality parameter. We consider the four cases, $\tau_{y} \in\{1,4,8,12\}$, and $\{\omega, r, \varpi\} \in$ $[0.1,10]$. Let us remark that for each value of $\{\omega, r, \varpi\} \in[0.1,10]$, we set it invariant for all time steps. Nevertheless, the computation of the theoretical optimal value of $\omega$ and $\varpi, \omega_{\text {opt }}$ and $\varpi_{\text {opt }}$, defined by (22) and (23), respectively, are variable in time. In order to compare the computational cost of BM algorithms with this optimal choice of the parameters, in Figure 15 we mark two horizontal lines at the level of the sum of the number of iterations in the duality loops at each time step obtained with $\mathrm{BM}\left(\omega_{\text {opt }}\right)$ and $\mathrm{BM}\left(\varpi_{\text {opt }}\right)$.

Let us remark that $\operatorname{BM}\left(\omega_{\text {opt }}\right)$ is slightly better than $\operatorname{BM}\left(\varpi_{\text {opt }}\right)$ for the case $\tau_{y}=1$, although both are close. Never- 


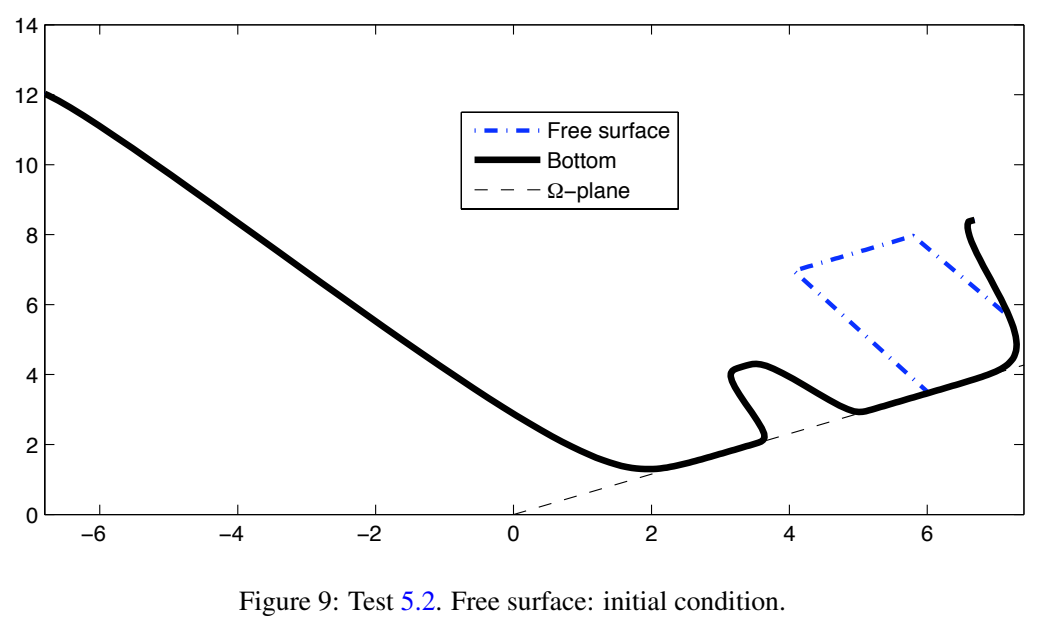

theless, for $\tau_{y} \in\{4,8,12\}$ there is a great difference of efficiency, being $\mathrm{BM}\left(\varpi_{\text {opt }}\right)$ close to the optimal computational cost in all situations. Finally, we remark that this difference is produced by the effect of the wet/dry fronts. In the case of numerical tests without wet/dry fronts, the behavior of both versions of BM method are very similar.

\section{Conclusions}

In this work, we proposed a discretization of a shallow Bingham model by a well-balanced finite volume method which is combined with duality techniques. Augmented Lagrangian and Bermúdez-Moreno algorithms have been considered to discretize the momentum equations. For the mass conservation equation we proposed a well-balanced correction which depends on the definition of the multiplier associated to the duality technique. This correction includes the use of a limiter that has been specially designed to recover the well-balanced properties of the numerical method. We prove that the proposed methods are able to preserve exactly two types of stationary solutions. A treatment of wet/dry fronts has also been proposed. It takes into account the rigidity of the material. For the case of the B.M. algorithm the definition of the optimal value of the parameter $\omega$ has been deduced. We also present two different versions of B.M. algorithm, by considering that $\omega$ can be variable in space. In the numerical tests section, we have first compared the algorithms for an analytical solution for a simplified model. Second, we have compared with the analytical solution of the multipliers for the case of a stationary solution. For the case of a random bottom a comparison of the multiplier with the theoretical one, which is proposed in Theorem 1, is also presented. Finally, we consider a test corresponding to an avalanche with an obstacle. This is a difficult test from a numerical point of view since it involves a complex geometry and wet/dry fronts together with strong viscoplasticity effects. In all these tests, we numerically show that computed velocities reach the stationary state. The proposed B.M.( $\varpi)$ algorithm, with $\omega$ variable in space, is in general the more efficient. The results corresponding to the proposed optimal choice of its parameter present a good agreement with the optimal computational cost.

As mentioned in the text, we will describe how to extend such schemes for 2D domains in a forthcoming article [17].

\section{Acknowledgments}

This research has been partially supported by the Spanish Government and FEDER through the Research projects MTM2009-07719, MTM2009-11923, MTM2012-38383-C02-01, MTM2012-38383-C02-02 and by the Andalusian government through the projects P11-FQM8179 and P11-RNM7069. Part of this work was done while P. V. was visiting E.D. F.-N. and J.M. G., during a stay in 2012 thanks to a grant from the Instituto Universitario de Investigación de Matemáticas de la Universidad de Sevilla (IMUS). P. V. wishes to thank everyone at IMUS for their hospitality. The support of French ANR Grant ANR-08-JCJC-0104 is also gratefully acknowledged. 


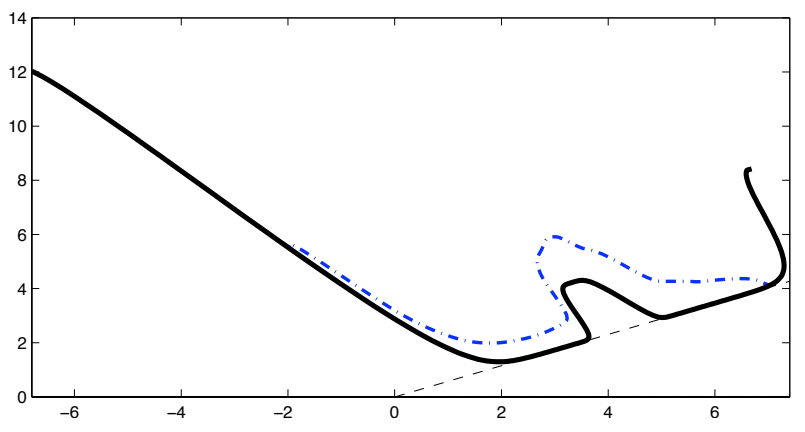

(a) $\tau_{y}=1$. Free surface.

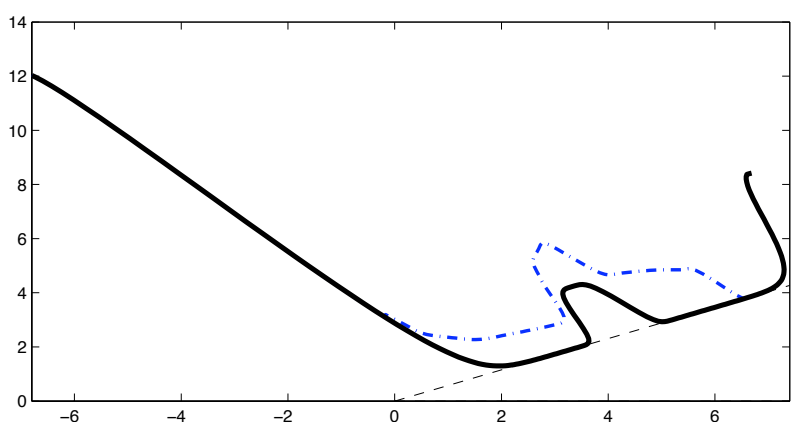

(c) $\tau_{y}=4$. Free surface.

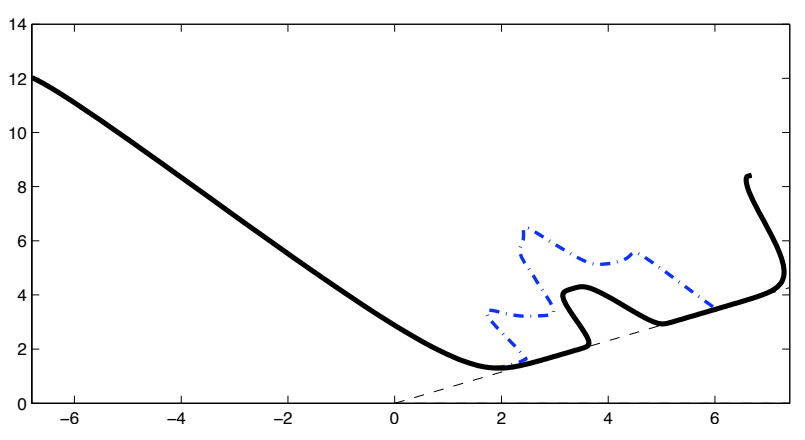

(e) $\tau_{y}=8$. Free surface.

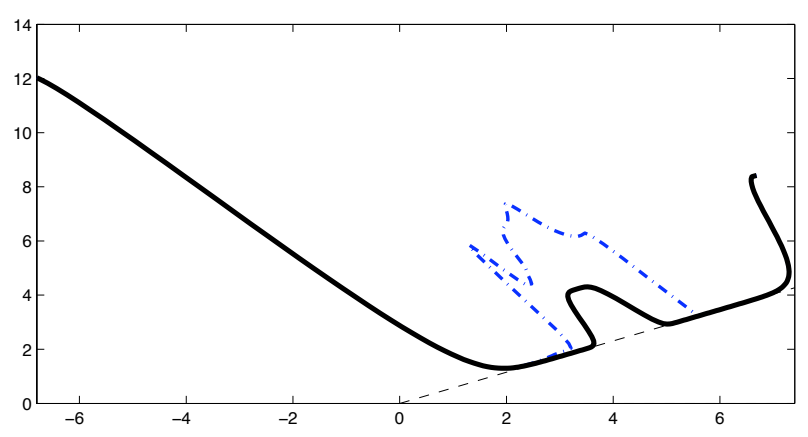

(g) $\tau_{y}=12$. Free surface.

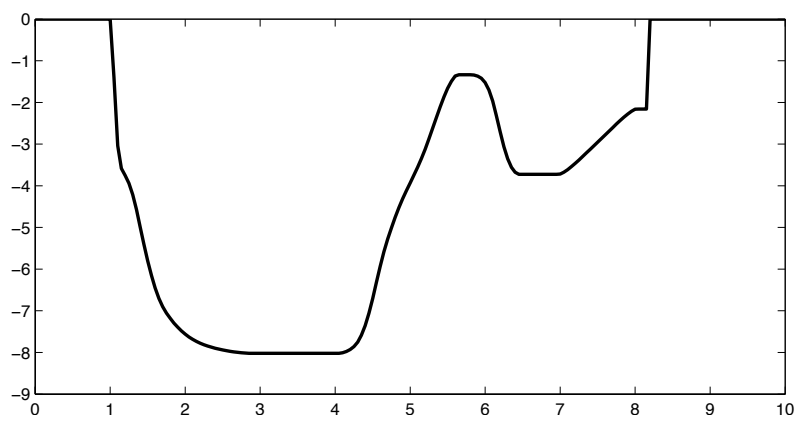

(b) $\tau_{y}=1$. Velocity.

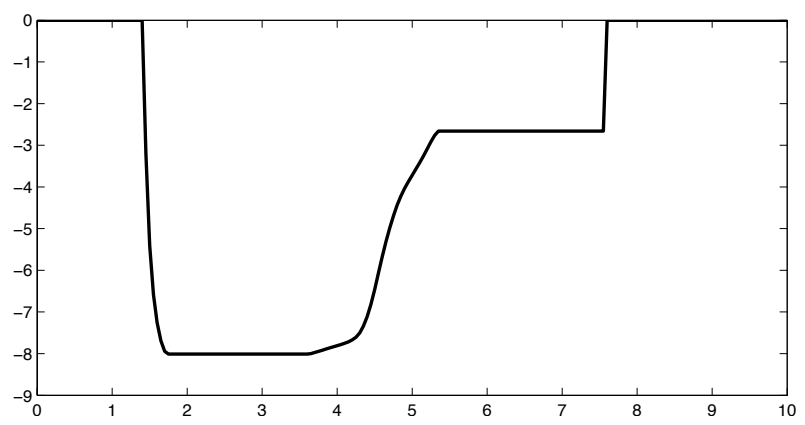

(d) $\tau_{y}=4$. Velocity.

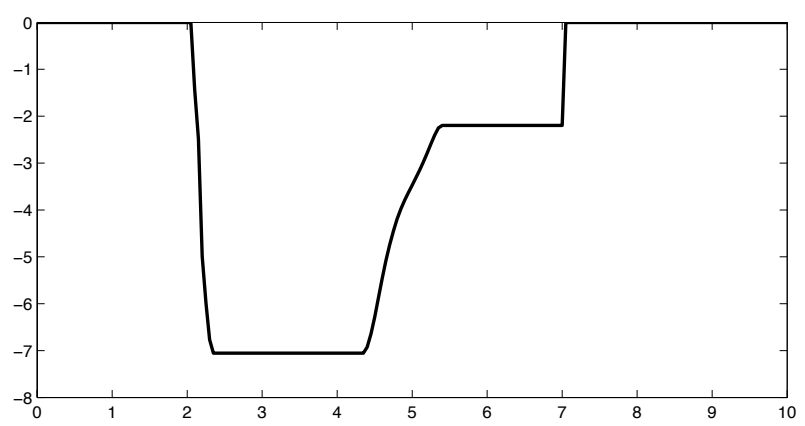

(f) $\tau_{y}=8$. Velocity.

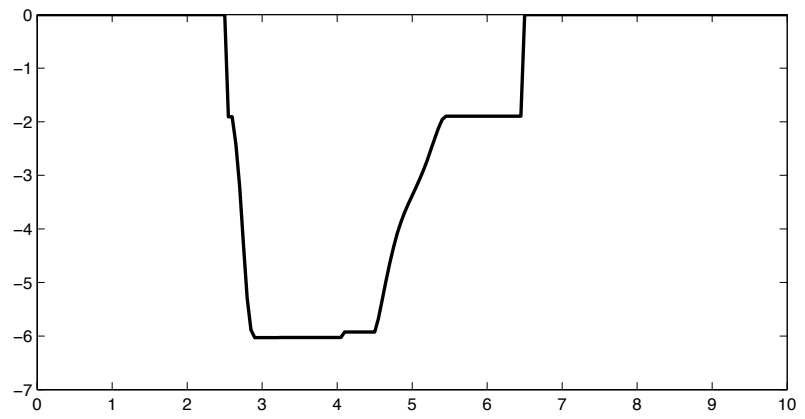

(h) $\tau_{y}=12$. Velocity.

Figure 10: Test 5.2. Free surface and velocity at $t=1 \mathrm{~s}$ for $\tau_{y} \in\{1,4,8,12\}$. 


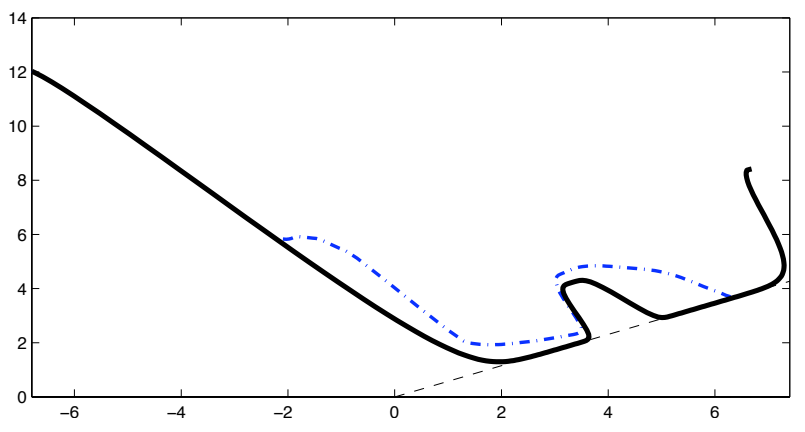

(a) $\tau_{y}=1$. Free surface.

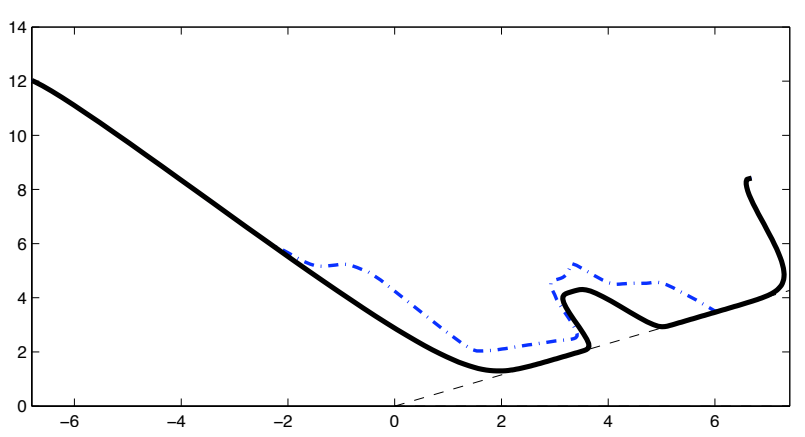

(c) $\tau_{y}=4$. Free surface.

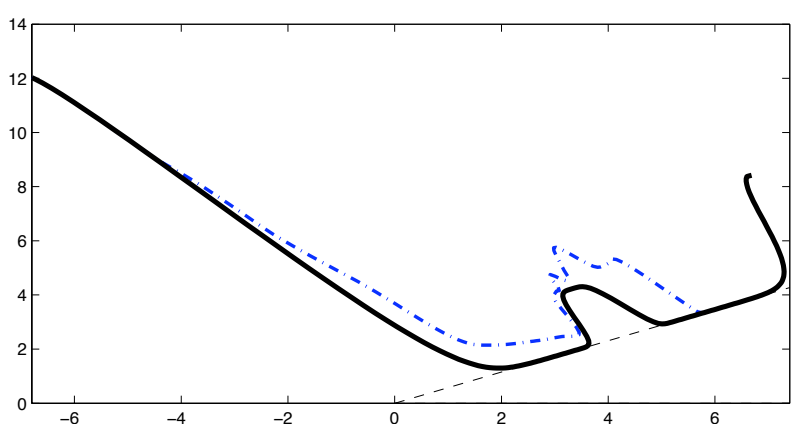

(e) $\tau_{y}=8$. Free surface.

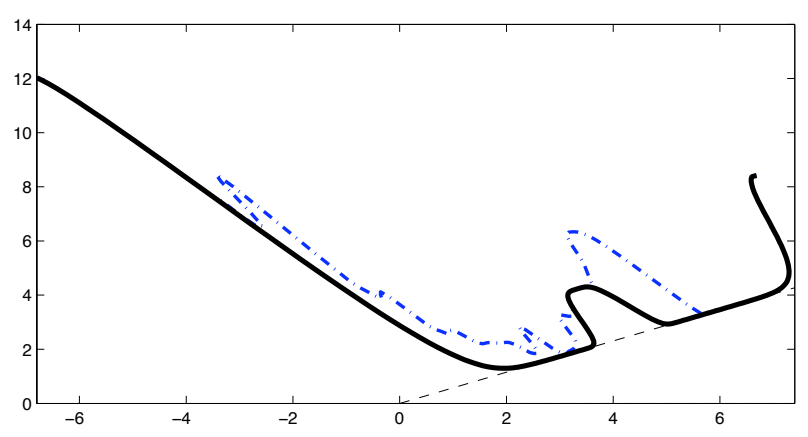

(g) $\tau_{y}=12$. Free surface.

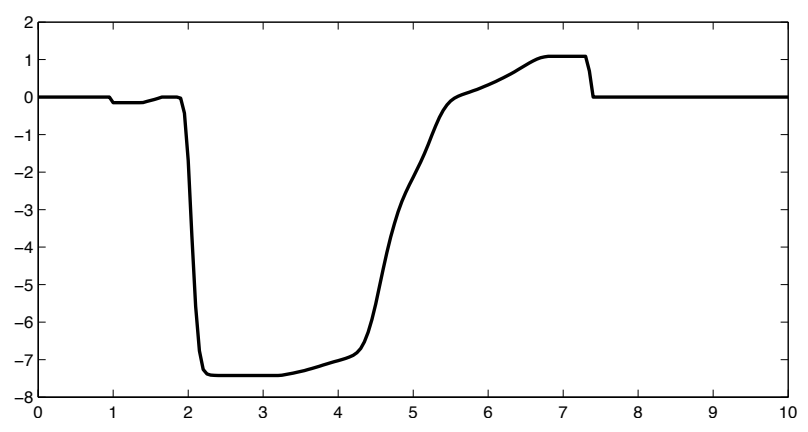

(b) $\tau_{y}=1$. Velocity.

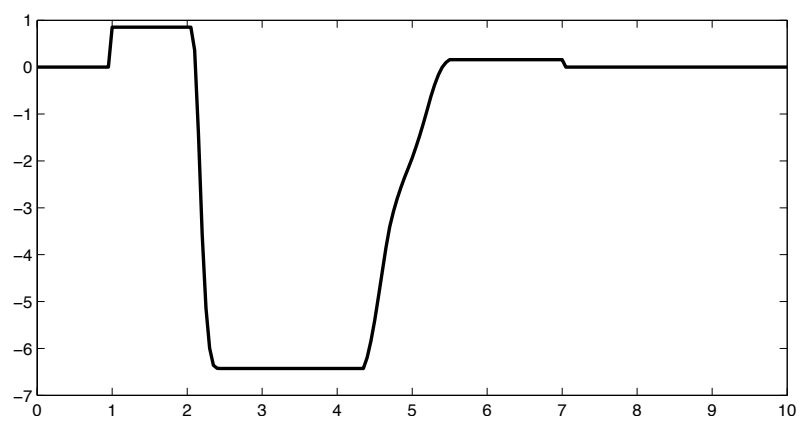

(d) $\tau_{y}=4$. Velocity.

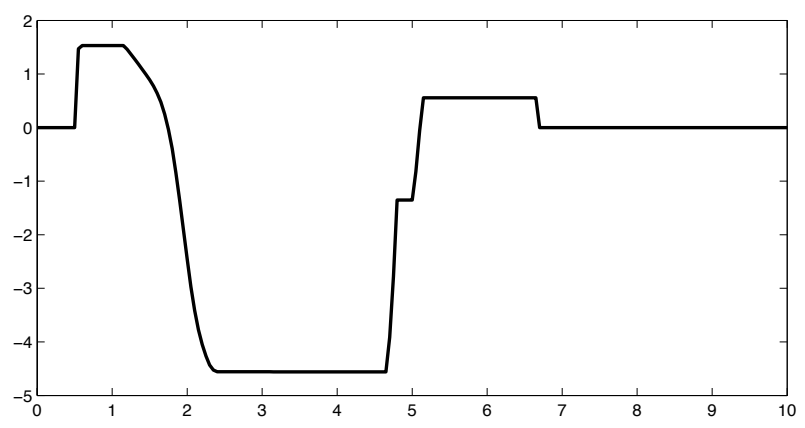

(f) $\tau_{y}=8$. Velocity.

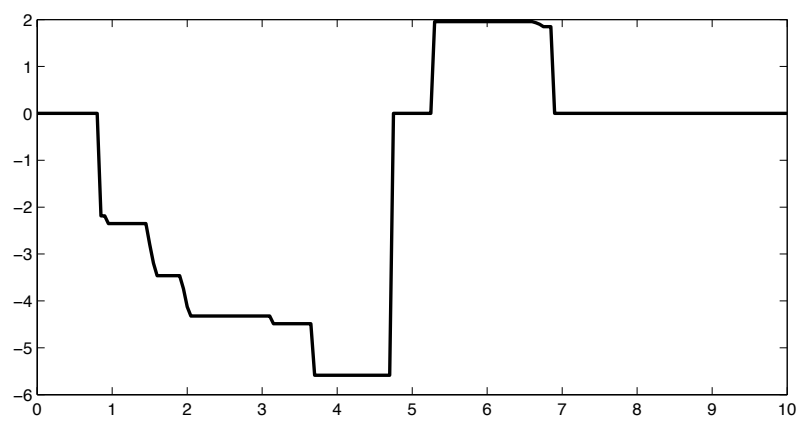

(h) $\tau_{y}=12$. Velocity

Figure 11: Test 5.2. Free surface and velocity at $t=1.5 \mathrm{~s}$ for $\tau_{y} \in\{1,4,8,12\}$. 


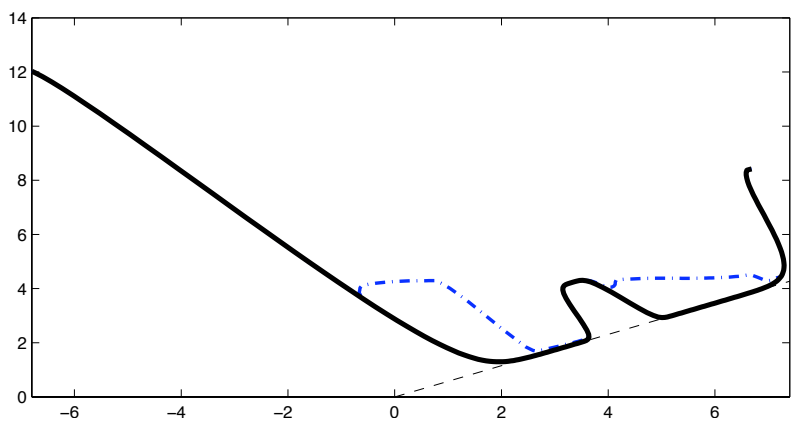

(a) $\tau_{y}=1$. Free surface.

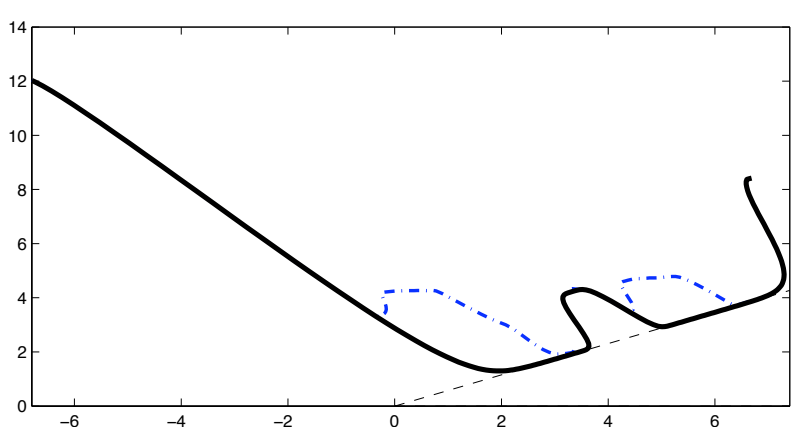

(c) $\tau_{y}=4$. Free surface.

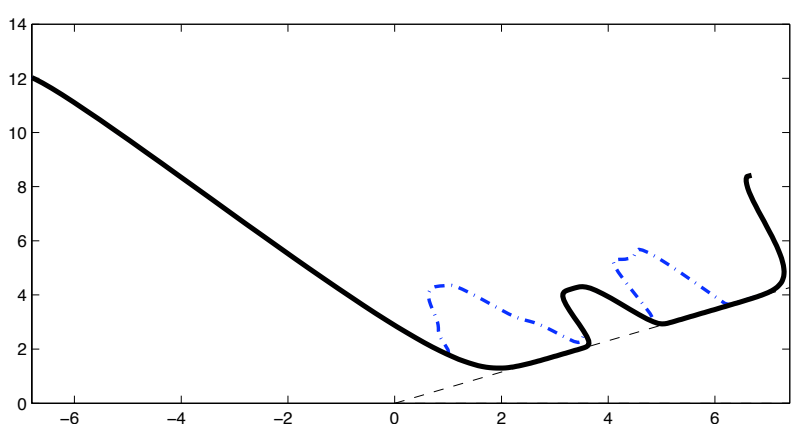

(e) $\tau_{y}=8$. Free surface.

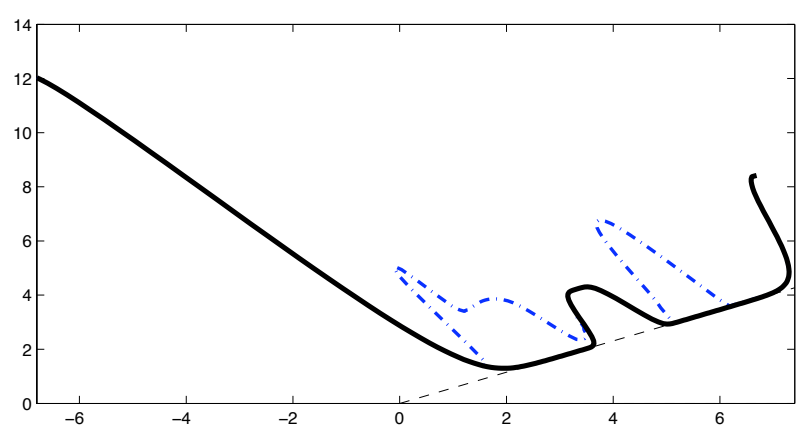

(g) $\tau_{y}=12$. Free surface.

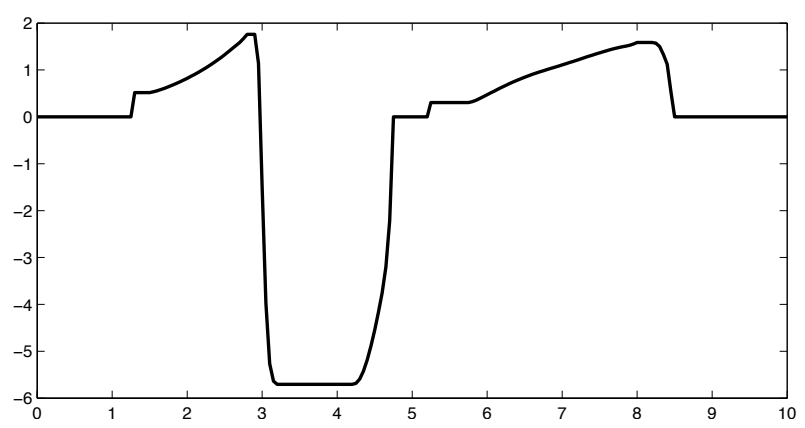

(b) $\tau_{y}=1$. Velocity.

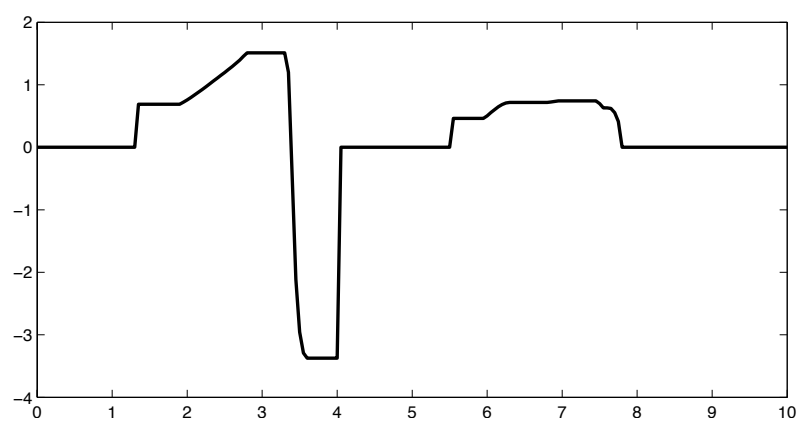

(d) $\tau_{y}=4$. Velocity.

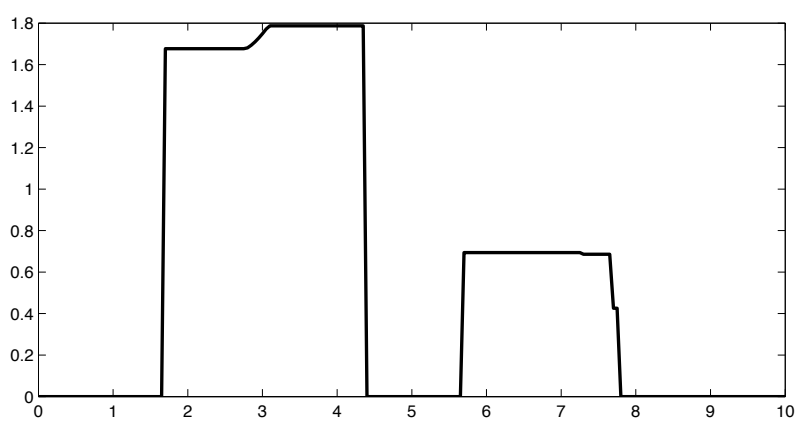

(f) $\tau_{y}=8$. Velocity.

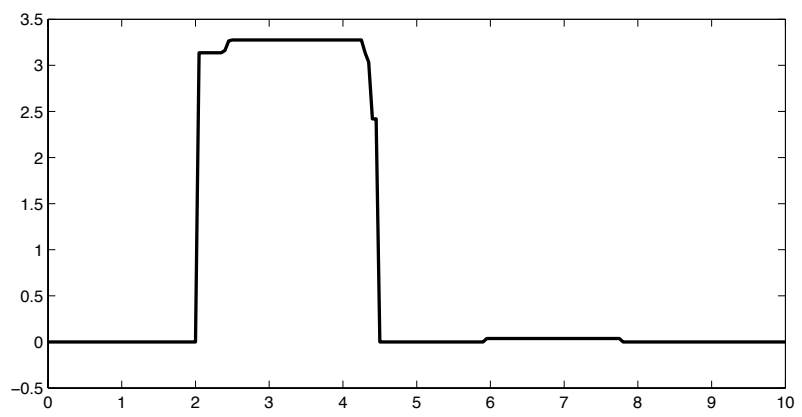

(h) $\tau_{y}=12$. Velocity.

Figure 12: Test 5.2. Free surface and velocity at $t=2 \mathrm{~s}$ for $\tau_{y} \in\{1,4,8,12\}$. 


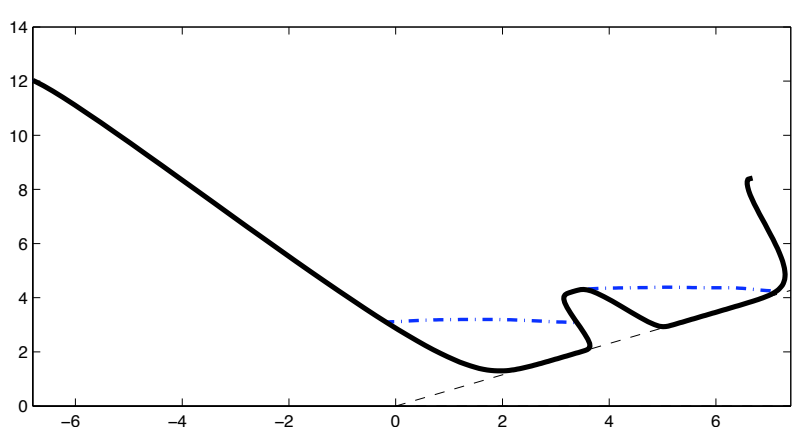

(a) $\tau_{y}=1$. Free surface.

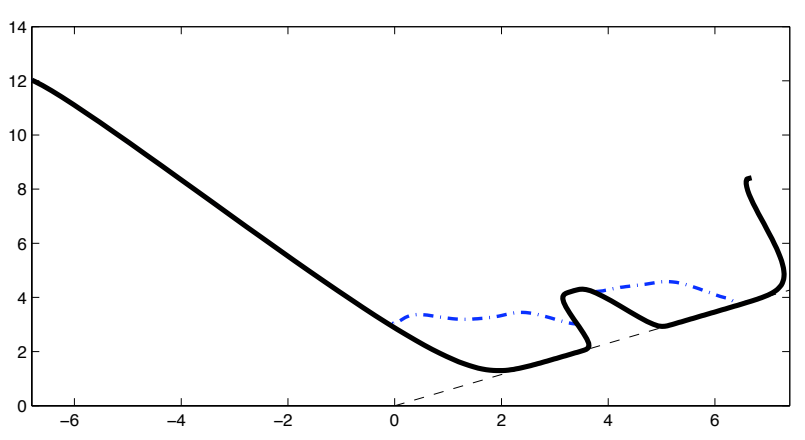

(c) $\tau_{y}=4$. Free surface.

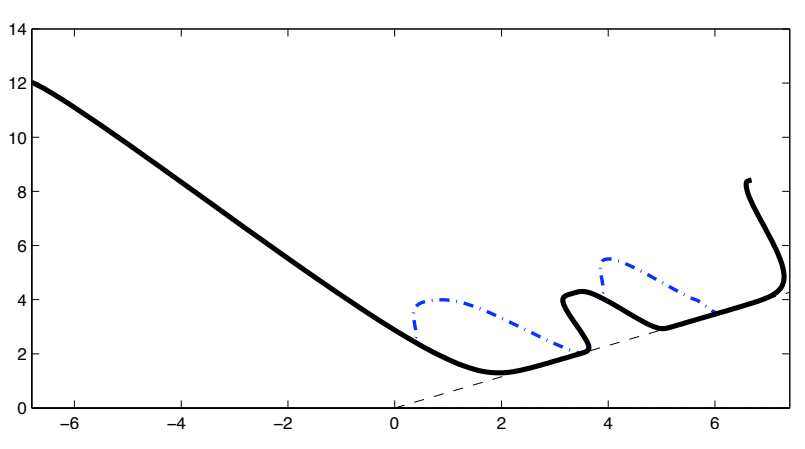

(e) $\tau_{y}=8$. Free surface.

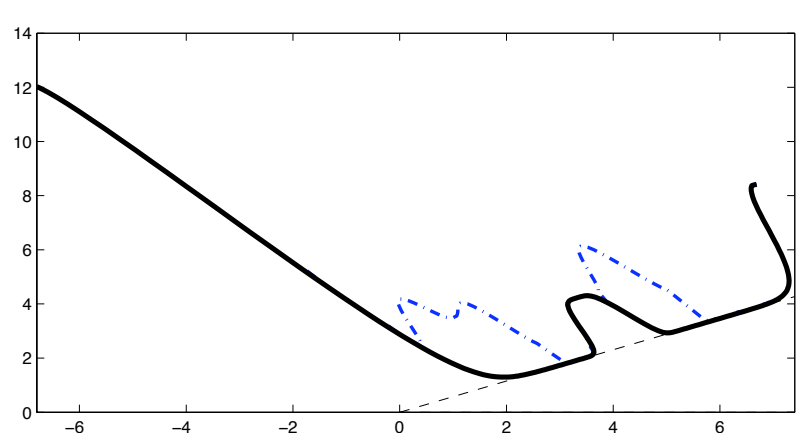

(g) $\tau_{y}=12$. Free surface.

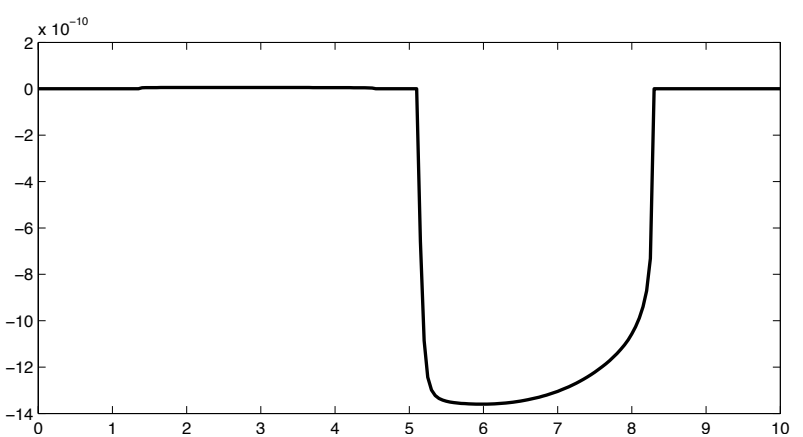

(b) $\tau_{y}=1$. Velocity.

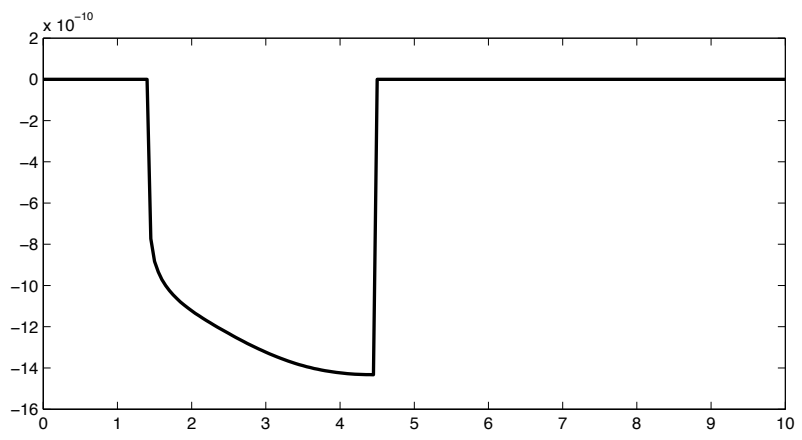

(d) $\tau_{y}=4$. Velocity.

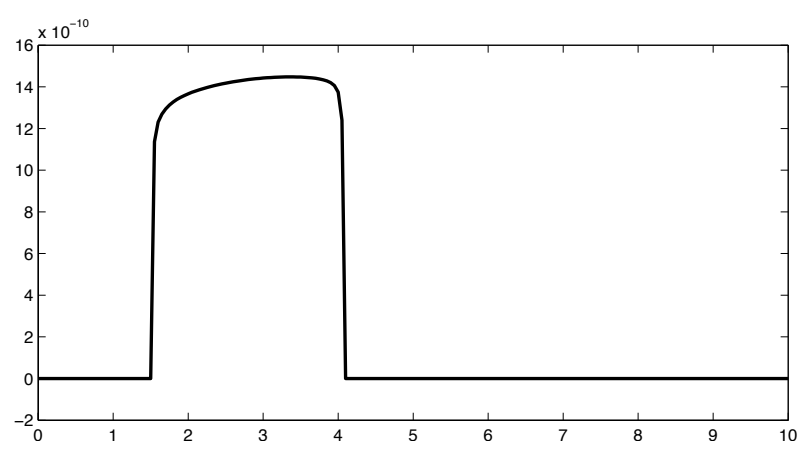

(f) $\tau_{y}=8$. Velocity.

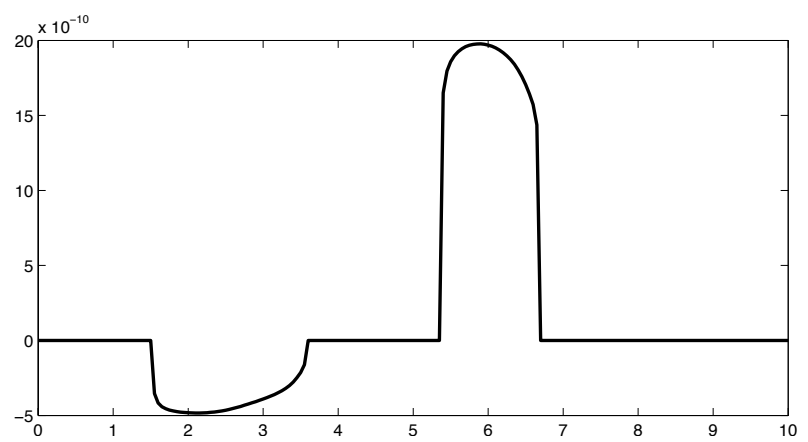

(h) $\tau_{y}=12$. Velocity.

Figure 13: Test 5.2. Free surface and velocity at rest at $t=24 \mathrm{~s}$ for $\tau_{y} \in\{1,4,8,12\}$. 


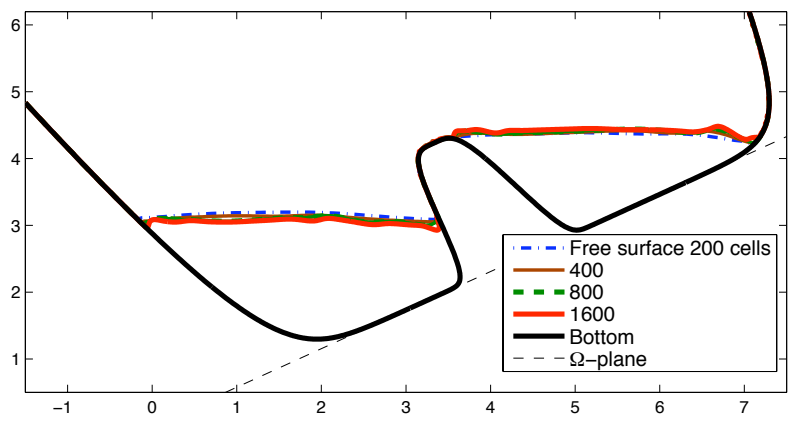

(a) $\tau_{y}=1$.

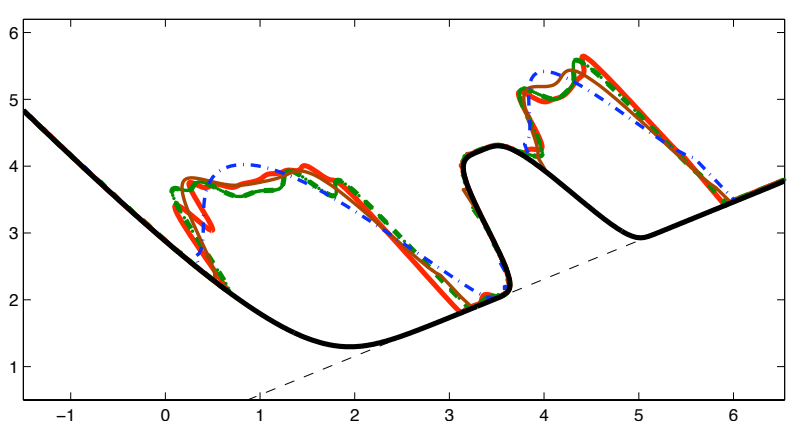

(c) $\tau_{y}=8$.

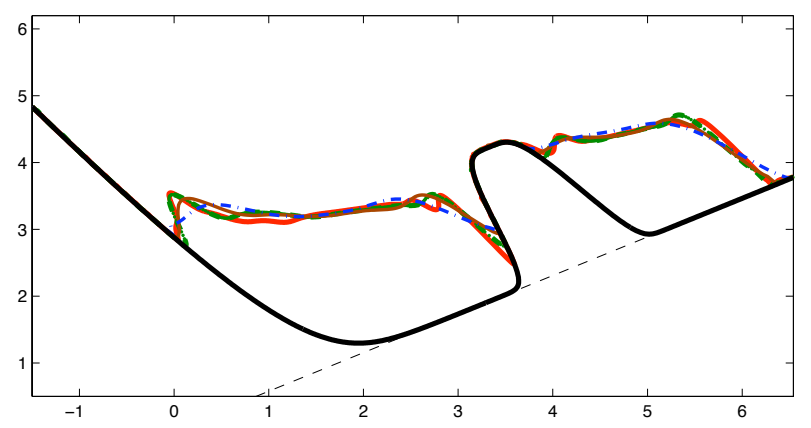

(b) $\tau_{y}=4$.

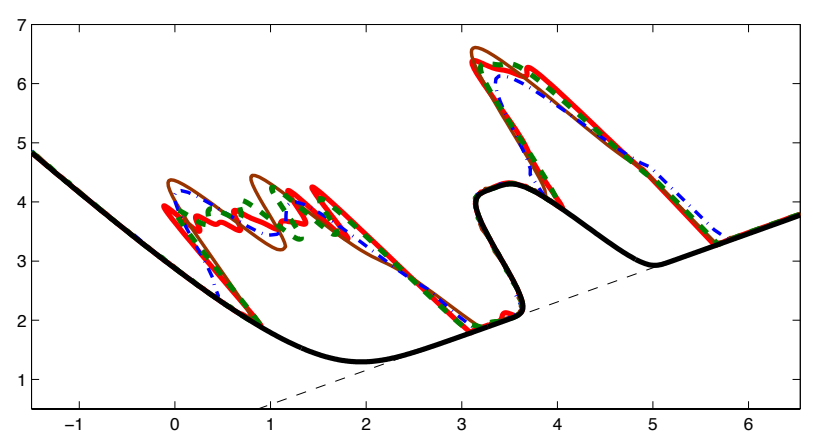

(d) $\tau_{y}=12$.

Figure 14: Test 5.2. Mesh refinement. Comparison of the free surface at rest (zooms) for 200, 400, 800 and 1600 computational cells. 


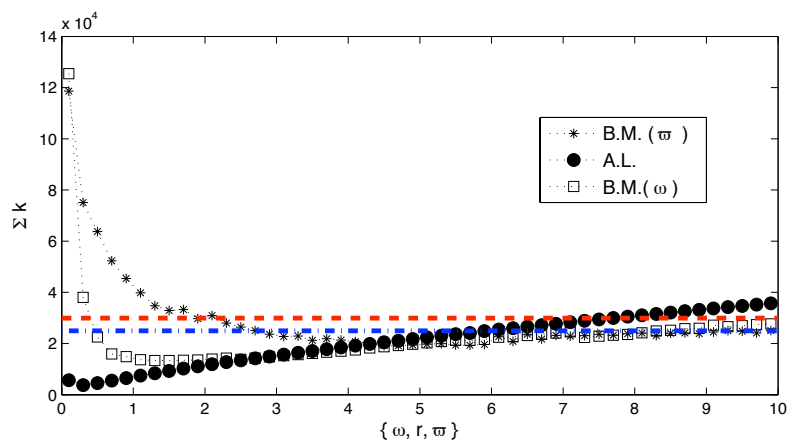

(a) $\tau_{y}=1$.

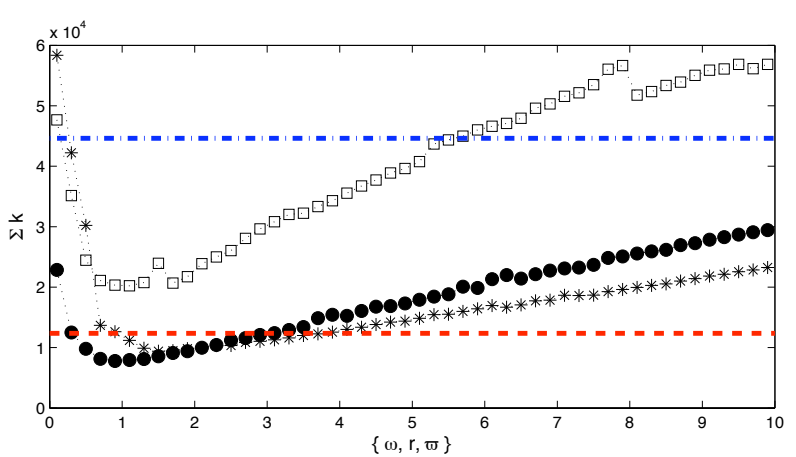

(c) $\tau_{y}=8$

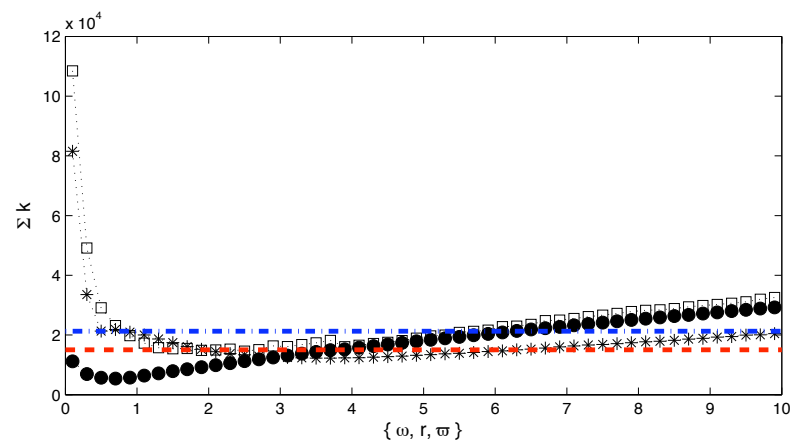

(b) $\tau_{y}=4$.

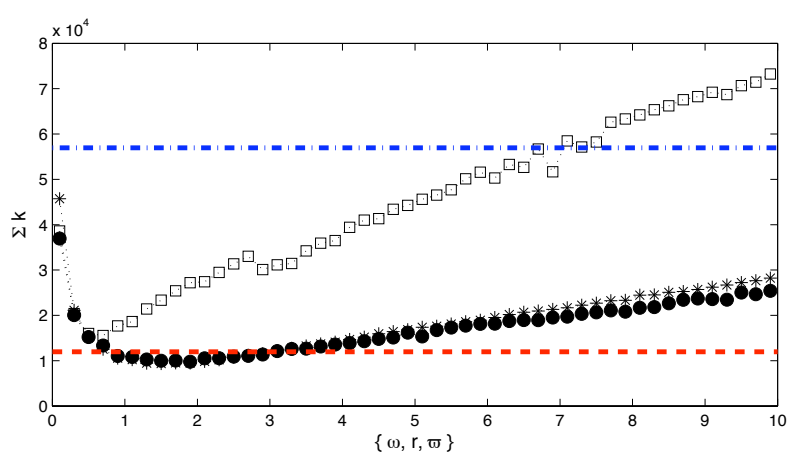

(d) $\tau_{y}=12$.

Figure 15: Test 5.2. Total number of iterations until $t=1$ for $\tau_{y} \in\{1,4,8,12\},\{\omega, r, \varpi\} \in[0.1,10]$. Red dashed line: total number of iterations for $\operatorname{BM}\left(\varpi_{\text {opt }}\right)$ defined by (23). Blue dashed-dot line: total number of iteration for BM $\left(\omega_{\text {opt }}\right)$ defined by $(22)$. 


\section{Appendix}

\section{Appendix A. Derivation of the AL approach}

Let us describe an Augmented Lagrangian method designed to solve problem (17) for $V^{n+1}$, supposing that $\left(H^{n}, V^{n}\right)$ are known. We refer to Glowinski and coworkers for details on this duality method à la Uzawa: the book [22] and the article [23] for a recent review in the context of Bingham flows.

The variational inequality (17) can be rewritten as a minimization problem:

$$
\mathcal{J}^{n}\left(V^{n+1}\right)=\min _{V \in \mathcal{V}} \mathcal{J}^{n}(V),
$$

where $\mathcal{J}^{n}(V)=F^{n}(B(V))+G^{n}(V)$, with $\mathcal{V}=H_{0}^{1}([0, L]), \mathcal{H}=L^{2}([0, L])$,

$$
B: \mathcal{V} \rightarrow \mathcal{H}, B(V)=\partial_{x} V, \quad F^{n}: \mathcal{H} \rightarrow \mathbb{R}, F^{n}(\lambda)=\int_{0}^{L} \tau_{y} \sqrt{2} H^{n}|\lambda| d x,
$$

and $G^{n}: \mathcal{V} \rightarrow \mathbb{R}$

$$
\begin{aligned}
G^{n}(V)= & \int_{0}^{L} H^{n}\left(\frac{V^{2} / 2-V^{n} V}{\Delta t}+\frac{1}{2} \partial_{x}\left(\left(V^{n}\right)^{2}\right) V\right) d x+\int_{0}^{L} \beta \frac{V^{2}}{2} d x \\
& +\int_{0}^{L} 4 \eta H^{n} \frac{1}{2}\left(\partial_{x} V\right)^{2} d x-\int_{0}^{L}\left(f_{\Omega}+f_{z} \partial_{x} b\right) H^{n} V+\int_{0}^{L} f_{z} \frac{\left(H^{n}\right)^{2}}{2} \partial_{x} V d x .
\end{aligned}
$$

As $\mathcal{J}^{n}(V)$ is a non-differentiable function, we consider the Lagrangian

$$
\begin{gathered}
\mathcal{L}^{n}: \mathcal{V} \times \mathcal{H} \times \mathcal{H} \rightarrow \mathbb{R}, \\
\mathcal{L}^{n}(V, q, \mu)=F^{n}(q)+G^{n}(V)+\int_{0}^{L} H^{n} \mu(B(V)-q) d x,
\end{gathered}
$$

and the Augmented Lagrangian function, for a given positive value $r \in \mathbb{R}$ :

$$
\mathcal{L}_{r}^{n}(V, q, \mu)=\mathcal{L}^{n}(V, q, \mu)+\frac{r}{2} \int_{0}^{L} H^{n}(B(V)-q)^{2} d x .
$$

Then, we search for the saddle point of $\mathcal{L}_{r}^{n}(V, q, \mu)$ over $\mathcal{V} \times \mathcal{H} \times \mathcal{H}$. Indeed, if we denote by $\left(V^{*}, q^{*}, \mu^{*}\right)$ this saddle point, then $V^{*}$ is the solution of the minimization problem (A.1) (cf. [22]). To do so, we consider the algorithm proposed in [22], based on Uzawa's algorithm, to approximate the saddle point of (A.2).

\section{Augmented Lagrangian algorithm}

- Initialization: Suppose that $V^{n}, H^{n}$ and $\mu^{n}$ are known. For $k=0$, we set $V^{k}=V^{n}$ and $\mu^{k}=\mu^{n}$. Initialize $r$.

- Iterate:

- Find $q^{k+1} \in \mathcal{H}$ solution of

$$
\mathcal{L}_{r}^{n}\left(V^{k}, q^{k+1}, \mu^{k}\right) \leq \mathcal{L}_{r}^{n}\left(V^{k}, \underline{q}, \mu^{k}\right), \quad \forall \underline{q} \in \mathcal{H} .
$$

In other words, $q^{k+1} \in \mathcal{H}$ is the solution of following minimization problem:

$$
\min _{\underline{q} \in \mathcal{H}}\left(\frac{H^{n} r}{2} \underline{q}^{2}+H^{n} \tau_{y} \sqrt{2}|\underline{q}|-H^{n}\left(\mu^{k}+r B\left(V^{k}\right)\right) \underline{q}\right) .
$$

The solution of this problem is (denoting the sign function as "sgn"):

$$
q^{k+1}= \begin{cases}0 & \text { if }\left|\mu^{k}+r B\left(V^{k}\right)\right|<\tau_{y}, \\ \frac{1}{r}\left(\left(\mu^{k}+r B\left(V^{k}\right)\right)-\tau_{y} \sqrt{2} \operatorname{sgn}\left(\mu^{k}+r B\left(V^{k}\right)\right)\right) & \text { otherwise. }\end{cases}
$$


- Find $V^{k+1} \in \mathcal{V}$ solution of

$$
\mathcal{L}_{r}^{n}\left(V^{k+1}, q^{k+1}, \mu^{k}\right) \leq \mathcal{L}_{r}^{n}\left(V, q^{k+1}, \mu^{k}\right), \quad \forall V \in \mathcal{V} .
$$

Thus, $V^{k+1}$ is the solution of a minimization problem, which can be characterized by differentiating $\mathcal{L}_{r}^{n}(V, q, \mu)$ with respect to $V$. From (A.2), we deduce that $V^{k+1}$ is the solution of the following linear problem (whose resolution is detailed in Section 4):

$$
\begin{gathered}
H^{n}\left(\frac{V^{k+1}-V^{n}}{\Delta t}\right)+\beta V^{k+1}-\partial_{x}\left(4 \eta H^{n} \partial_{x}\left(V^{k+1}\right)\right)-\partial_{x}\left(r H^{n} \partial_{x}\left(V^{k+1}\right)\right) \\
=\left(f_{\Omega}+f_{z} \partial_{x} b\right) H^{n}+\partial_{x}\left(f_{z} \frac{\left(H^{n}\right)^{2}}{2}\right)-\frac{H^{n}}{2} \partial_{x}\left(\left(V^{n}\right)^{2}\right)+\partial_{x}\left(H^{n}\left(\mu^{k}-r q^{k+1}\right)\right) .
\end{gathered}
$$

- Update the Lagrange multiplier via

$$
\mu^{k+1}=\mu^{k}+r\left(\partial_{x} V^{k+1}-q^{k+1}\right)
$$

- Check convergence (see below) and update: $V^{k}=V^{k+1}, \mu^{k}=\mu^{k+1}, k \mapsto k+1$ and go to the next iteration...

- ... until convergence is reached:

$$
\frac{\left\|\mu^{k+1}-\mu^{k}\right\|}{\left\|\mu^{k}\right\|} \leq \text { tol. }
$$

At convergence, we get the value of $V^{n+1}$ by setting $V^{n+1}=V^{k+1}$ (in the numerical tests presented in this paper, we set tol $=10^{-5}$ ). It is shown in [22] that this algorithm converges to the saddle point of (A.2).

Of note, we did not describe the discretization in space yet. As we said previously, we want to adopt a finite volume approach. Consequently, it is worth realizing that the underlying global problem coupling (16) and (17) involves the following system (we use a slight change of notation which will be useful in the following: $H^{n+1}$ is denoted as $H^{k+1}$; in spite of this choice, note again that $H^{k+1}$ is not involved in the Augmented Lagrangian algorithm and, so, does not change in this loop):

$$
(P)^{n, k}\left\{\begin{array}{l}
\frac{H^{k+1}-H^{n}}{\Delta t}+\partial_{x}\left(H^{n} V^{n}\right)=0 \\
H^{n}\left(\frac{V^{k+1}-V^{n}}{\Delta t}\right)+\beta V^{k+1}-\partial_{x}\left(4 \eta H^{n} \partial_{x}\left(V^{k+1}\right)\right)-\partial_{x}\left(r H^{n} \partial_{x}\left(V^{k+1}\right)\right) \\
=\left(f_{\Omega}+f_{z} \partial_{x} b\right) H^{n}+\partial_{x}\left(\frac{\left(H^{n}\right)^{2} f_{z}}{2}\right)-\frac{H^{n}}{2} \partial_{x}\left(\left(V^{n}\right)^{2}\right)+\partial_{x}\left(H^{n}\left(\mu^{k}-r q^{k+1}\right)\right) .
\end{array}\right.
$$

Consequently, even if there is a decoupling of both problems in terms of the time discretization and the Augmented Lagrangian algorithm, it appears that to obtain a global well-balanced scheme, there must be a coupling between the mass and momentum equations induced by the source terms (involving topography and the Lagrange multiplier). For shallow water type systems with source terms, this has been extensively studied in the literature. In Section 4, we precisely describe the aforementioned coupling for the present problem.

The natural follow-up of this appendix is Section 3.1.

\section{Appendix B. Derivation of the BM method}

In this section, the solution of the velocity problem (17) is approached by means of the duality algorithm introduced in [4]. We shall focus on the application of the method to our particular case and refer to [4], [31], [32] and [20] for further details. 
Define $\mathcal{V}=H_{0}^{1}([0, L])$ and let $\langle\cdot, \cdot\rangle$ be the duality pairing between $\mathcal{V}$ and its dual space $\mathcal{V}^{\prime}=H^{-1}([0, L])$. The variational inequality (17) can be rewritten as: Find $V \in \mathcal{V}$ such that

$$
\langle A(V), \Psi-V\rangle+j(\Psi)-j(V) \geq\langle L, \Psi-V\rangle
$$

holds for every $\Psi \in \mathcal{V}$. Here, $A: \mathcal{V} \rightarrow \mathcal{V}^{\prime}$ denotes the linear operator

$$
\langle A(V), \Psi\rangle=\int_{0}^{L}\left[\left(\frac{H^{n}}{\Delta t}+\beta\right) V-\partial_{x}\left(4 \eta H^{n} \partial_{x} V\right)\right] \Psi d x, \quad \Psi \in \mathcal{V}
$$

Throughout this section it will be assumed that there exists a constant $H_{\min }^{n}$ such that $H^{n}(x) \geq H_{\min }^{n}>0$. Then the operator $A$ is coercive with constant

$$
\gamma=\min \left(\frac{H_{\min }^{n}}{\Delta t}+\beta, 4 \eta H_{\min }^{n}\right)>0 .
$$

The functional $j: \mathcal{V} \rightarrow \mathbb{R}$ is defined by

$$
j(V)=\int_{0}^{L} \Phi(x, B(V)(x)) d x
$$

where $\Phi:[0, L] \times \mathbb{R} \rightarrow \mathbb{R}$ is given by $\Phi(x, z)=\tau_{y} \sqrt{2} H^{n}(x)|z|$, and $B: \mathcal{V} \rightarrow \mathcal{H}$ is the derivative operator $B(V)=\partial_{x} V$, where $\mathcal{H}=L^{2}([0, L])$ with the usual scalar product $(\cdot, \cdot)_{L^{2}}$ and norm $\|\cdot\|_{L^{2}}$. Finally, $L \in \mathcal{V}^{\prime}$ represents the functional

$$
L=H^{n} \frac{V^{n}}{\Delta t}-\frac{H^{n}}{2} \partial_{x}\left(\left(V^{n}\right)^{2}\right)+H^{n}\left(f_{\Omega}+f_{z} \partial_{x} b\right)+\frac{1}{2} \partial_{x}\left(\left(H^{n}\right)^{2} f_{z}\right)
$$

Notice that $j(V)=T(B(V))$, where $T: \mathcal{H} \rightarrow \mathbb{R}$ is given by

$$
T(Z)=\int_{0}^{L} \Phi(x, Z(x)) d x .
$$

Let $\omega>0$ be an arbitrary parameter and define $G^{\omega}=\partial T-\omega I$, where the subdifferential $\partial T$ is the multivalued operator

$$
\partial T(Z)=\{W \in \mathcal{H}: T(Q)-T(Z) \geq(W, Q-Z), \forall Q \in \mathcal{H}\}, \quad Z \in \mathcal{H} .
$$

As $T$ is proper, convex and lower semicontinuous, its subdifferential turns out to be a maximal monotone operator. Thus, if $\lambda>0$ is such that $\lambda \omega<1$, the resolvent $J_{\lambda}=\left(I+\lambda G^{\omega}\right)^{-1}$ is an univalued operator defined on $\mathcal{H}$. Moreover, if $\lambda \omega \leq 1 / 2$ the Yosida approximation of $G^{\omega}$,

$$
G_{\lambda}^{\omega}=\frac{I-J_{\lambda}^{\omega}}{\lambda}
$$

is a Lipschitz function with constant $1 / \lambda$ (see [9]).

Observe that, due to the continuity of $T$, the subdifferentials of $j$ and $T$ are related as ([16])

$$
\partial j(V)=B^{*}(\partial T(B(V))),
$$

where $B^{*}$ denotes the dual operator of $B$. Thus, problem (B.1) can be reformulated as follows: Find $V \in \mathcal{V}$ and $\theta \in \mathcal{H}$ such that

$$
\left\{\begin{array}{l}
A(V)+B^{*}(\omega B(V))+B^{*}(\theta)=L, \\
\theta=G_{\lambda}^{\omega}(B(V)+\lambda \theta) .
\end{array}\right.
$$

As $G_{\lambda}^{\omega}$ is a Lipschitz function, it makes then sense to define the fixed point Bermúdez-Moreno algorithm as follows: For $k \geq 0, \theta^{k}$ being known, compute $V^{k}$ and $\theta^{k+1}$ by solving

$$
\left\{\begin{array}{l}
A\left(V^{k}\right)+B^{*}\left(\omega B\left(V^{k}\right)\right)+B^{*}\left(\theta^{k}\right)=L, \\
\theta^{k+1}=G_{\lambda}^{\omega}\left(B\left(V^{k}\right)+\lambda \theta^{k}\right) .
\end{array}\right.
$$


As it was proved in [4], the sequence $V^{k}$ converges to the solution $V$ due to the coerciveness of the operator $A$. For questions regarding the convergence of the multipliers $\theta^{k}$ we refer the reader to [31].

The key point in the definition of the BM algorithm (B.4) is the construction of the Yosida approximation $G_{\lambda}^{\omega}$, that will be worked out in what follows. First of all, notice that ([16])

$$
\partial T(Z)=\{W \in \mathcal{H}: W(x) \in \partial \Phi(x, Z(x)) \text { a.e. } x \in[0, L]\}
$$

Thus, a simple computation shows that $\partial T(Z)$ is the set of elements $W \in \mathcal{H}$ that verify

$$
W(x) \in \begin{cases}\left\{\tau_{y} \sqrt{2} H^{n}(x)\right\} & \text { if } Z(x)>0, \\ {\left[-\tau_{y} \sqrt{2} H^{n}(x), \tau_{y} \sqrt{2} H^{n}(x)\right]} & \text { if } Z(x)=0, \\ \left\{-\tau_{y} \sqrt{2} H^{n}(x)\right\} & \text { if } Z(x)<0,\end{cases}
$$

a.e. $x \in[0, L]$. After some algebra, we deduce the following expression, which is valid a.e. $x \in[0, L]$ for each $Z \in \mathcal{H}$ :

$$
G_{\lambda}^{\omega}(Z)(x)= \begin{cases}\frac{-\omega Z(x)+\tau_{y} \sqrt{2} H^{n}(x)}{1-\lambda \omega} & \text { if } Z(x)>\lambda \tau_{y} \sqrt{2} H^{n}(x), \\ \frac{Z(x)}{\lambda} & \text { if } Z(x) \in\left[-\lambda \tau_{y} \sqrt{2} H^{n}(x), \lambda \tau_{y} \sqrt{2} H^{n}(x)\right], \\ \frac{-\omega Z(x)-\tau_{y} \sqrt{2} H^{n}(x)}{1-\lambda \omega} & \text { if } Z(x)<-\lambda \tau_{y} \sqrt{2} H^{n}(x) .\end{cases}
$$

The complete algorithm for solving (17) is then given in Section 3.1.1 of the main text.

\section{Appendix C. Study of the BM optimal parameter}

Given a uniform partition of $[0, L]$ of size $h$, let $\mathcal{V}_{h}$ be the finite-dimensional subspace of $\mathcal{V}$ of standard conforming $\mathbb{P}_{1}$ finite elements. Consider now the discrete versions of (B.3) and (B.4), where the elements $V$ and $V^{k}$ are assumed to belong to $\mathcal{V}_{h}$ instead of $\mathcal{V}$ (for the sake of clarity, the dependence on $h$ will not be explicitly stated). Combining both expressions, we get

$$
\left\langle A\left(V^{k}-V\right), \Psi\right\rangle+\omega\left(\partial_{x} V^{k}-\partial_{x} V, \partial_{x} \Psi\right)_{L^{2}}+\left(\theta^{k}-\theta, \partial_{x} \Psi\right)_{L^{2}}=0, \quad \forall \Psi \in \mathcal{V}_{h},
$$

and also, using that $G_{\lambda}^{\omega}$ is Lipschitz with constant $1 / \lambda$,

$$
\begin{aligned}
\left\|\theta^{k+1}-\theta\right\|_{L^{2}}^{2} & =\left\|G_{\lambda}^{\omega}\left(\partial_{x} V^{k}+\lambda \theta^{k}\right)-G_{\lambda}^{\omega}\left(\partial_{x} V+\lambda \theta\right)\right\|_{L^{2}}^{2} \\
& \leq \frac{1}{\lambda^{2}}\left\|\partial_{x} V^{k}-\partial_{x} V\right\|_{L^{2}}^{2}+\left\|\theta^{k}-\theta\right\|_{L^{2}}^{2}+\frac{2}{\lambda}\left(\theta^{k}-\theta, \partial_{x} V^{k}-\partial_{x} V\right)_{L^{2}} .
\end{aligned}
$$

Taking $\Psi=V^{k}-V$ in (C.1), we deduce

$$
\left\langle A\left(V^{k}-V\right), V^{k}-V\right\rangle+\omega\left\|\partial_{x} V^{k}-\partial_{x} V\right\|_{L^{2}}^{2}+\left(\theta^{k}-\theta, \partial_{x} V^{k}-\partial_{x} V\right)_{L^{2}}=0,
$$

so (C.2) can be written as

$$
\left\|\theta^{k+1}-\theta\right\|_{L^{2}}^{2} \leq\left\|\theta^{k}-\theta\right\|_{L^{2}}^{2}-4 \omega\left\langle A\left(V^{k}-V\right), V^{k}-V\right\rangle,
$$

taking into account that $\lambda \omega=1 / 2$.

Assume that $\gamma_{1}$ and $\gamma_{2}$ are positive constants (which may depend on $h$ ) verifying

$$
\gamma_{1}\|\Psi\|_{L^{2}} \leq\left\|\partial_{x} \Psi\right\|_{L^{2}} \leq \gamma_{2}\|\Psi\|_{L^{2}}, \quad \forall \Psi \in \mathcal{V}_{h},
$$

and define $H_{\max }^{n}=\left\|H^{n}\right\|_{\infty}$. Then, from (C.1) and the definition of $A$, we have

$$
\begin{aligned}
\left(\theta^{k}-\theta, \partial_{x} \Psi\right)_{L^{2}} & =-\left\langle A\left(V^{k}-V\right), \Psi\right\rangle-\omega\left(\partial_{x} V^{k}-\partial_{x} V, \partial_{x} \Psi\right)_{L^{2}} \\
& \leq\left(\frac{H_{\max }^{n}}{\Delta t}+\beta\right)\left\|V^{k}-V\right\|_{L^{2}}\|\Psi\|_{L^{2}}+\left(4 \eta H_{\max }^{n}+\omega\right)\left\|\partial_{x} V^{k}-\partial_{x} V\right\|_{L^{2}}\left\|\partial_{x} \Psi\right\|_{L^{2}} \\
& \leq\left[\left(\frac{H_{\max }^{n}}{\Delta t}+\beta\right) \gamma_{1}^{-1}+\left(4 \eta H_{\max }^{n}+\omega\right) \gamma_{2}\right]\left\|V^{k}-V\right\|_{L^{2}}\left\|\partial_{x} \Psi\right\|_{L^{2}}, \quad \forall \Psi \in \mathcal{V}_{h} .
\end{aligned}
$$


Taking now $\Psi \in \mathcal{V}_{h}$ such that $\partial_{x} \Psi=\theta^{k}-\theta$, we obtain from the above inequality

$$
\left\|\theta^{k}-\theta\right\|_{L^{2}} \leq\left[\left(\frac{H_{\max }^{n}}{\Delta t}+\beta\right) \gamma_{1}^{-1}+\left(4 \eta H_{\max }^{n}+\omega\right) \gamma_{2}\right]\left\|V^{k}-V\right\|_{L^{2}}
$$

On the other hand, using the coerciveness of $A$, we have that

$$
\begin{aligned}
\left\langle A\left(V^{k}-V\right), V^{k}-V\right\rangle & \geq \gamma\left\|V^{k}-V\right\|_{V}^{2}=\gamma\left(\left\|V^{k}-V\right\|_{L^{2}}^{2}+\left\|\partial_{x} V^{k}-\partial_{x} V\right\|_{L^{2}}^{2}\right) \\
& \geq \gamma\left(1+\gamma_{1}^{2}\right)\left\|V^{k}-V\right\|_{L^{2}}^{2},
\end{aligned}
$$

where the coercivity constant $\gamma$ was given by (B.2).

Finally, combining (C.3), (C.5) and (C.6), we deduce the following inequality:

$$
\left\|\theta^{k+1}-\theta\right\|_{L^{2}} \leq \mathcal{L}(\omega)\left\|\theta^{k}-\theta\right\|_{L^{2}}
$$

where

$$
\mathcal{L}(\omega)=\left\{1-4 \omega \gamma\left(1+\gamma_{1}^{2}\right)\left[\left(\frac{H_{\max }^{n}}{\Delta t}+\beta\right) \gamma_{1}^{-1}+\left(4 \eta H_{\max }^{n}+\omega\right) \gamma_{2}\right]^{-2}\right\}^{1 / 2}
$$

The optimal choice of the parameter $\omega_{\text {opt }}$ will be that minimizing $\mathcal{L}(\omega)$. An easy computation shows that

$$
\omega_{\mathrm{opt}}=\left(\frac{H_{\max }^{n}}{\Delta t}+\beta\right) \frac{1}{\gamma_{1} \gamma_{2}}+4 \eta H_{\max }^{n} .
$$

From a practical point of view, it is necessary to have good estimates of the constants $\gamma_{1}$ and $\gamma_{2}$ appearing in (C.4). To this end, we consider the following spectral problem: Find $0 \neq v_{h} \in \mathcal{V}_{h}$ and $\mu_{h} \in \mathbb{R}$ such that

$$
\left(v_{h}^{\prime}, \varphi_{h}^{\prime}\right)_{L^{2}}=\mu_{h}\left(v_{h}, \varphi_{h}\right)_{L^{2}}, \quad \forall \varphi_{h} \in \mathcal{V}_{h}
$$

It is well-known ([6]) that, for uniform mesh size $h$, there exists an orthonormal basis of $\mathcal{V}_{h}$ composed by eigenvectors $\left\{\varphi_{h}^{(1)}, \ldots, \varphi_{h}^{(N)}\right\}$ associated to the eigenvalues $0<\mu_{h}^{(1)} \leq \cdots \leq \mu_{h}^{(N)}$ of the spectral problem. Indeed, these eigenvalues have the following form:

$$
\mu_{h}^{(j)}=\frac{6}{h^{2}} \frac{1-\cos (j \pi h / L)}{2+\cos (j \pi h / L)}, \quad j=1, \ldots, N
$$

where $N$ denotes the number of internal nodes. For arbitrary $v_{h}=\sum_{j=1}^{N} v_{j} \varphi_{h}^{(j)}$ we have

$$
\left\|\partial_{x} v_{h}\right\|_{L^{2}}^{2}=\left(\partial_{x} v_{h}, \partial_{x} v_{h}\right)_{L^{2}}=\sum_{j=1}^{N} v_{j}^{2}\left(\partial_{x} \varphi_{h}^{(j)}, \partial_{x} \varphi_{h}^{(j)}\right)_{L^{2}}=\sum_{j=1}^{N} v_{j}^{2} \mu_{h}^{(j)}\left(\varphi_{h}^{(j)}, \varphi_{h}^{(j)}\right)_{L^{2}}\left\{\begin{array}{l}
\geq \mu_{h}^{(1)}\left\|v_{h}\right\|_{L^{2}}^{2}, \\
\leq \mu_{h}^{(N)}\left\|v_{h}\right\|_{L^{2}}^{2},
\end{array}\right.
$$

so we deduce the inequalities

$$
\sqrt{\mu_{h}^{(1)}}\left\|v_{h}\right\|_{L^{2}} \leq\left\|\partial_{x} v_{h}\right\|_{L^{2}} \leq \sqrt{\mu_{h}^{(N)}}\left\|v_{h}\right\|_{L^{2}}, \quad \forall v_{h} \in \mathcal{V}_{h}
$$

As noticed in [6], the following optimal estimate holds as $h \rightarrow 0$ :

$$
\left|\mu^{(j)}-\mu_{h}^{(j)}\right|=O\left(h^{2}\right)
$$

where $\mu^{(j)}=(j \pi / L)^{2}$. Therefore, we consider the approximations $\gamma_{1}=\sqrt{\mu^{(1)}}=\pi / L$ and $\gamma_{N}=\sqrt{\mu^{(N)}}=N \pi / L$.

Summarizing, a quasi-optimal choice of the parameter $\omega$ would be given by

$$
\omega_{\mathrm{opt}}\left(H_{\max }^{n}\right)=\left(\frac{H_{\max }^{n}}{\Delta t}+\beta\right) \frac{L^{2}}{N \pi^{2}}+4 \eta H_{\max }^{n}
$$

In the case of wet/dry fronts further adaptations can be made and are described in Section 3.1.3. 


\section{Appendix D. The coupled scheme in the case of the $A L$}

For this duality method the structure of the complete algorithm is very close to the BM one. In particular, the linear problem verified by the speed in the duality loop ([Step 2] below) is embedded in the general formulation (39)-(43).

\section{Global numerical scheme for (13)-(14) - Augmented Lagrangian method}

- Initialization at time $t=0$ for $n=0: V^{n}, H^{n}, \mu^{n}$ are given by the initial conditions.

- Time loop: For $n=0, \ldots, n_{\max }$.

\section{- Resolution of the problem on $V^{k+1}$}

$\left\{V_{i}^{n}\right\}_{i},\left\{H_{i}^{n}\right\}_{i}$ and $\left\{\mu_{i+1 / 2}^{n}\right\}_{i}$ are known.

Compute quantities which are invariant in the following loop:

\section{Augmented Lagrangian loop:}

[Step 0] Initialize for $k=0$ : for all $i, V_{i}^{k}=V_{i}^{n}$ and $\mu_{i+1 / 2}^{k}=\mu_{i+1 / 2}^{n}$.

[Step 1] Update $\left\{q_{i+1 / 2}^{k+1}\right\}_{i}$ by computing

$$
q_{i+1 / 2}^{k+1}=\frac{1}{r}\left(\mu_{i+1 / 2}^{k}+r \frac{V_{i+1}^{k}-V_{i}^{k}}{\Delta x}\right)\left(1-\frac{\tau_{y} \sqrt{2}}{\left|\mu_{i+1 / 2}^{k}+r \frac{V_{i+1}^{k}-V_{i}^{k}}{\Delta x}\right|}\right)_{+} .
$$

In the case of a wet/dry front, if $H_{i} \geq H_{\varepsilon}, H_{i+1}<H_{\varepsilon}$ and $b_{i}+H_{i}<b_{i+1}$ then we set $q_{i+1 / 2}^{k+1}=0$. Or if $H_{i}<H_{\varepsilon}, H_{i+1} \geq H_{\varepsilon}$ and $b_{i+1}+H_{i+1}<b_{i}$, we also set $q_{i+1 / 2}^{k+1}=0$.

[Step 2] Update $\left\{V_{i}^{k+1}\right\}_{i}$ by solving the linear system defined by the second component of (29). See details at (39)-(43), with $\delta_{i+1 / 2}^{n}$ and $\zeta_{i+1 / 2}^{k}$ given by (28)-(a) and (27)-(a).

[Step 3] Update $\left\{\mu_{i+1 / 2}^{k+1}\right\}_{i}$ via

$$
\mu_{i+1 / 2}^{k+1}=\mu_{i+1 / 2}^{k}+r\left(\frac{V_{i+1}^{k+1}-V_{i}^{k+1}}{\Delta x}-q_{i+1 / 2}^{k+1}\right) .
$$

[Step 4] Set: for all $i, V_{i}^{k}=V_{i}^{k+1}, \mu_{i+1 / 2}^{k}=\mu_{i+1 / 2}^{k+1}$ and return to Step 1 .

[Step 5] At convergence, when condition (A.7) is verified, set $V_{i}^{n+1}=V_{i}^{k+1}$ and $\mu_{i+1 / 2}^{n+1}=\mu_{i+1 / 2}^{k+1} \forall i$.

- Resolution of the problem on $H^{k+1}$

Compute $H^{n+1}=H^{k+1}$ with the finite volume method determined by the first component of (29), defined in terms of the most recent Lagrange multiplier $\left\{\mu_{i+1 / 2}^{n+1}\right\}_{i}$ and taking into account the wet/dry treatment presented before where needed.

This can be compared to the Bermúdez-Moreno algorithm presented in Section 4.1.

\section{Appendix E. Proof of Theorem 1}

\section{Proof}

It is enough to prove that if for a time $t=t^{n}$ we have $H_{i}^{n}=H_{i}^{0}$ and $V_{i}^{n}=0$, then $H_{i}^{n+1}=H_{i}^{n}$ and $V_{i}^{n+1}=0, \forall$ $i=1, \ldots, M$.

i) First, let us prove that the numerical scheme preserves exactly the stationary solution defined by $V_{i}^{n}=0, b_{i}+H_{i}^{n}=$ constant, where $\left\{H_{i}^{n}\right\}_{i}=\left\{H_{i}^{0}\right\}_{i}$ verifies (47).

i.a) First, let us prove that $V_{i}^{n+1}=0$, where $V_{i}^{n+1}=V_{i}^{k_{\text {end }}}$, being $k_{\text {end }}$ the number of iterations necessary to converge in the iterative algorithm. If we prove that $\boldsymbol{b}^{n, 1}=0$ (see equation (40)), as $\left\{V_{j}^{1}\right\}_{j}$ is the solution of the linear system (39), it implies that $V_{i}^{1}=0$. Moreover, if we prove that $\zeta_{i+1 / 2}^{2}=\zeta_{i+1 / 2}^{1}$ then we obtain that $k_{\text {end }}=1$ and $V_{i}^{n+1}=0$. Thus, let us prove that $\boldsymbol{b}^{n, 1}=0$. 
The components of the vector $\boldsymbol{b}^{n, 1}=0$ are

$$
\boldsymbol{b}_{i}^{n, 1}=\boldsymbol{b}_{i}^{n,(1)}+\boldsymbol{b}_{i}^{n, 1,(2)}+\boldsymbol{b}_{i}^{n, 1,(3)}, \quad i=1, \ldots, M .
$$

where $\boldsymbol{b}_{i}^{n,(1)}, \boldsymbol{b}_{i}^{n, 1,(2)}$ and $\boldsymbol{b}_{i}^{n, 1,(3)}$ are defined by (41), (42) and (43), respectively.

Taking into account that $\zeta_{i+1 / 2}^{0}$ is defined by (48) and $b_{i}+H_{i}^{0}=$ constant, we have that

$$
\boldsymbol{b}_{i}^{n, 1,(2)}=\frac{\zeta_{i+1 / 2}^{k}-\zeta_{i-1 / 2}^{k}}{\Delta x}=-H_{i}^{0} f_{\Omega}
$$

As $V_{i}^{0}=0$ and $\boldsymbol{b}_{i}^{n, 1,(3)}$ is defined in terms of the second component of numerical flux function (31), we have

$$
\boldsymbol{b}_{i}^{n, 1,(3)}=H_{i}^{0} f_{z} \frac{H_{i+1}^{0}-H_{i-1}^{0}}{2 \Delta x} .
$$

Then, taking into account the definition of $\boldsymbol{b}_{i}^{n,(1)}(41)$ we obtain that

$$
\boldsymbol{b}_{i}^{n, 1}=\frac{H_{i}^{0} f_{z}}{2 \Delta x}\left(b_{i+1}+H_{i+1}^{0}-\left(b_{i-1}+H_{i-1}^{0}\right)\right)=0 .
$$

Thus, $\boldsymbol{b}^{n, 1}=0$ and consequently $V_{i}^{1}=0, i=1, \ldots, M$. Let us now prove that $\zeta_{i+1 / 2}^{2}=\zeta_{i+1 / 2}^{1} \cdot \zeta_{i+1 / 2}^{1}$ is defined by (48), and by (47) we have that $\left|\zeta_{i+1 / 2}^{1}\right| \leq \tau_{y} \sqrt{2} H_{i+1 / 2}^{0}$. Then (see Subsection 4.1) for the AL algorithm we have that $\left|\mu_{j+1 / 2}^{1}\right| \leq \tau_{y} \sqrt{2}$. So, by (D.1) we have that $q_{i+1 / 2}^{2}=0$ and consequently, since $V_{j}^{1}=0 \forall j$, by (D.2) we obtain that $\mu_{i+1 / 2}^{2}=\mu_{i+1 / 2}^{1}$. For the BM algorithm we have that $\theta_{i+1 / 2}^{1} \leq \tau_{y} \sqrt{2} H_{j+1 / 2}^{0}$; then, by (38) we have that $\theta_{j+1 / 2}^{2}=\theta_{j+1 / 2}^{1}$. Therefore, we deduce that $\zeta_{i+1 / 2}^{2}=\zeta_{i+1 / 2}^{1}$.

Consequently, we obtain that $V_{i}^{n+1}=V_{i}^{n}=0$.

i.b) Now, let us prove that $H_{i}^{n+1}=H_{i}^{n}$. From (29), and taking into account that $V_{j}^{n}=0$ for $j=i-1, i, i+1$, we have that

$$
\begin{aligned}
H_{i}^{n+1}=H_{i}^{n}+\frac{1}{2} \frac{\Delta t}{\Delta x} \quad\left(\alpha _ { 0 , i + 1 / 2 } \left(H_{i+1}^{n}-H_{i}^{n}+\left[\mathcal{G}^{n}\left(\left\{\zeta_{j+1 / 2}^{k}\right\}_{j=i-1}^{j=i+1}\right]_{1}\right)-\right.\right. \\
\alpha_{0, i-1 / 2}\left(H_{i}^{n}-H_{i-1}^{n}+\left[\mathcal{G}^{n}\left(\left\{\zeta_{j+1 / 2}^{k}\right\}_{j=i-2}^{j=i}\right]_{1}\right)\right) .
\end{aligned}
$$

Then, it is enough to prove that

$$
H_{i+1}^{n}-H_{i}^{n}+\left[\mathcal{G}^{n}\left(\left\{\zeta_{j+1 / 2}^{k}\right\}_{j=i-1}^{j=i+1}\right]_{1}=0, \quad \forall i\right.
$$

where

$$
\left[\mathcal{G}^{n}\left(\left\{\zeta_{j+1 / 2}^{k}\right\}_{j=i-1}^{j=i+1}\right]_{1}=\frac{1}{f_{z}}\left(f_{\Omega} \Delta x+f_{z}\left(b_{i+1}-b_{i}\right)+\frac{\Delta\left(\zeta+\delta^{n} \partial_{x} V\right)_{i+1 / 2}^{k}}{H_{i+1 / 2}}\right) .\right.
$$

In this case, as $b_{i}+H_{i}^{n}$ is constant, the flux limiter used in the definition of $\Delta\left(\zeta+\delta^{n} \partial_{x} V\right)_{i+1 / 2}^{k}$ is equal to one. So, we obtain

$$
\Delta\left(\zeta+\delta^{n} \partial_{x} V\right)_{i+1 / 2}^{k}=\frac{1}{2}\left(\zeta_{i+3 / 2}^{k_{\text {end }}}-\zeta_{i-1 / 2}^{k_{\text {end }}}\right) .
$$

By using that $\zeta_{j+1 / 2}^{k_{\text {end }}}=\zeta_{j+1 / 2}^{1}$ (see Section i.a) of the proof), for $j=i-1$ and $j=i+1$, and (48), we obtain that

$$
\Delta\left(\zeta+\delta^{n} \partial_{x} V\right)_{i+1 / 2}^{k}=-\Delta x H_{i+1 / 2}^{n} f_{\Omega} .
$$

As a consequence, $\left[\mathcal{G}^{n}\left(\left\{\zeta_{j+1 / 2}^{k}\right\}_{j=i-1}^{j=i+1}\right]_{1}=b_{i+1}-b_{i}\right.$ and

$$
H_{i+1}^{n}-H_{i}^{n}+\left[\mathcal{G}^{n}\left(\left\{\zeta_{j+1 / 2}^{k}\right\}_{j=i-1}^{j=i+1}\right]_{1}=H_{i+1}+b_{i+1}-\left(H_{i}+b_{i}\right)=0 .\right.
$$


Then, $H_{i}^{n+1}=H_{i}^{n}$, which concludes the proof for case $i$ ).

ii) For the case of a stationary solution with a horizontal free surface, we suppose that

$$
f_{\Omega} x_{i}+f_{z}\left(H_{i}^{0}+b_{i}\right)=\text { constant } .
$$

First, note that using (48) and (E.1) we have

$$
\zeta_{i+1 / 2}^{1}=-\left(\sum_{j=1}^{i} H_{j}^{0}-\sum_{j=1}^{[M / 2]} H_{j}^{0}\right)\left(f_{\Omega} x_{i+1}+f_{z}\left(H_{i+1}^{0}+b_{i+1}\right)-\left(f_{\Omega} x_{i}+f_{z}\left(H_{i}^{0}+b_{i}\right)\right)\right)=0 .
$$

As a consequence, we have that

$$
\boldsymbol{b}_{i}^{n, 1}=\frac{H_{i}^{0}}{2 \Delta x}\left(f_{\Omega} x_{i+1}+f_{z}\left(H_{i+1}^{0}+b_{i+1}\right)-\left(f_{\Omega} x_{i}+f_{z}\left(H_{i}^{0}+b_{i}\right)\right)\right)=0,
$$

and

$$
H_{i+1}^{n}-H_{i}^{n}+\left[\mathcal{G}^{n}\left(\left\{\zeta_{j+1 / 2}^{k}\right\}_{j=i-1}^{j=i+1}\right]_{1}=\frac{1}{f_{z}}\left(f_{\Omega} x_{i+1}+f_{z}\left(H_{i+1}^{0}+b_{i+1}\right)-\left(f_{\Omega} x_{i}+f_{z}\left(H_{i}^{0}+b_{i}\right)\right)\right)=0 .\right.
$$

By (E.2), (E.3), (E.4) and following the steps of Section $i$ ) of the proof, we obtain that $H_{i}^{n+1}=H_{i}^{n}$ and $V_{i}^{n+1}=0$.

Then follows, in the main text, the numerical tests of Section 5.

\section{Appendix F. A duct flow case}

This test is inspired by the classical (Newtonian) Poiseuille flow between two infinite parallel plates (orthogonal to the $x$-axis). The flow thus depends only on the transverse variable $x$ and is defined by the velocity $V=V(x)$ in the direction parallel to the plates. Here, instead of Navier-Stokes, we consider a Bingham constitutive law. To recover the so-called Bingham-Poiseuille flow from (14), we suppose that the height is constant $(H=1)$, the flow is stationary (no time dependence) and "laminar" (no "convective" term), and that there is no friction $(\beta=0)$ ). Finally, the force needs to be transformed to model this pressure driven flow: this is easily achieved by taking $\alpha=\pi / 2$, which leads to a remaining force which is $f_{\Omega}$ (simply denoted $f$ in the following) and has to be exactly thought as the pressure gradient which drives the flow. We use a relaxation formulation of the aforementioned model by using the following degenerate version of (14):

$$
\forall \Psi, \int_{0}^{L} \partial_{t} V(\Psi-V)+4 \eta \partial_{x}(V) \partial_{x}(\Psi-V)+\tau_{y} \sqrt{2}\left(\left|\partial_{x} \Psi\right|-\left|\partial_{x} V\right|\right) d x \geq \int_{0}^{L} f(\Psi-V) d x .
$$

Here $t$ is not a physical time but a relaxation time; the solution of (F.1) converges, for $t \rightarrow+\infty$, to the solution of the aforementioned Bingham-Poiseuille flow which is known analytically:

$$
V_{B P}(\chi)=\frac{f}{8 \eta} \begin{cases}\left(\frac{L}{2}-\chi_{y}\right)^{2} & \text { if } 0 \leq \chi \leq \chi_{y}, \\ \left(\frac{L}{2}-\chi_{y}\right)^{2}-\left(\chi-\chi_{y}\right)^{2} & \text { if } \chi_{y}<\chi \leq \frac{L}{2} .\end{cases}
$$

where $\chi=\left|x-\frac{L}{2}\right|, \chi_{y}=\frac{\tau_{y}}{f}$ locates the yield zone and the domain is defined for $x \in[0, L]$. Note that, $L$ and $\tau_{y}$ being given, if $f \leq \frac{2 \tau_{y}}{L}$, then $V_{B P} \equiv 0$ : if the pressure gradient is too small, the driving force is not sufficient to overcome the yield stress and the material remains rigid.

The interest of this test is that, though rather simplified, the velocity is not null and the resulting problem contains all the mathematical difficulties of the Bingham model. We want to check the ability of the numerical methods presented previously to converge to the stationary solution (F.2) and proceed as follows. We take a null initial condition:

$$
\forall x \in[0, L], V(t=0, x)=0,
$$


and homogeneous Dirichlet boundary conditions:

$$
\forall t \geq 0, V(t, 0)=V(t, L)=0 .
$$

We set a domain of length $L=1$, discretized with 200 points. Moreover, $\eta=0.2, \tau_{y}=4 / \sqrt{2}$ and $f=25$. We compute the evolution of the solution and consider that a numerical stationary solution has been reached when the relative error between two iterations in time (with a time step $\Delta t=0.05$ ) is smaller than $10^{-8}$, namely

$$
\frac{\left\|V^{n+1}-V^{n}\right\|_{1}}{\left\|V^{n+1}\right\|_{1}}<10^{-8}
$$

The convergence of the velocity (resp. multiplier) to the stationary solution is shown in Figure F.16 (resp. F.17) for both Augmented Lagrangian and Bermúdez-Moreno methods. Indeed, for this test, we can easily compute the multipliers associated to $V_{B P}$ (they are known up to a constant); for the Augmented Lagrangian method we have

$$
\mu(x)=-f x-4 \eta \partial_{x} V_{B P}+c s t,
$$

whereas for the Bermúdez-Moreno method it reads

$$
\theta(x)=-f x-(4 \eta+\omega) \partial_{x} V_{B P}+c s t .
$$

In both cases, to have an enlightening graphical representation of the numerical multiplier and the analytical one, we determine the constant in such a way the curves are superimposed at convergence. A good qualitative convergence is observed on Figures F.16 and F.17 and this is confirmed quantitatively in the following.
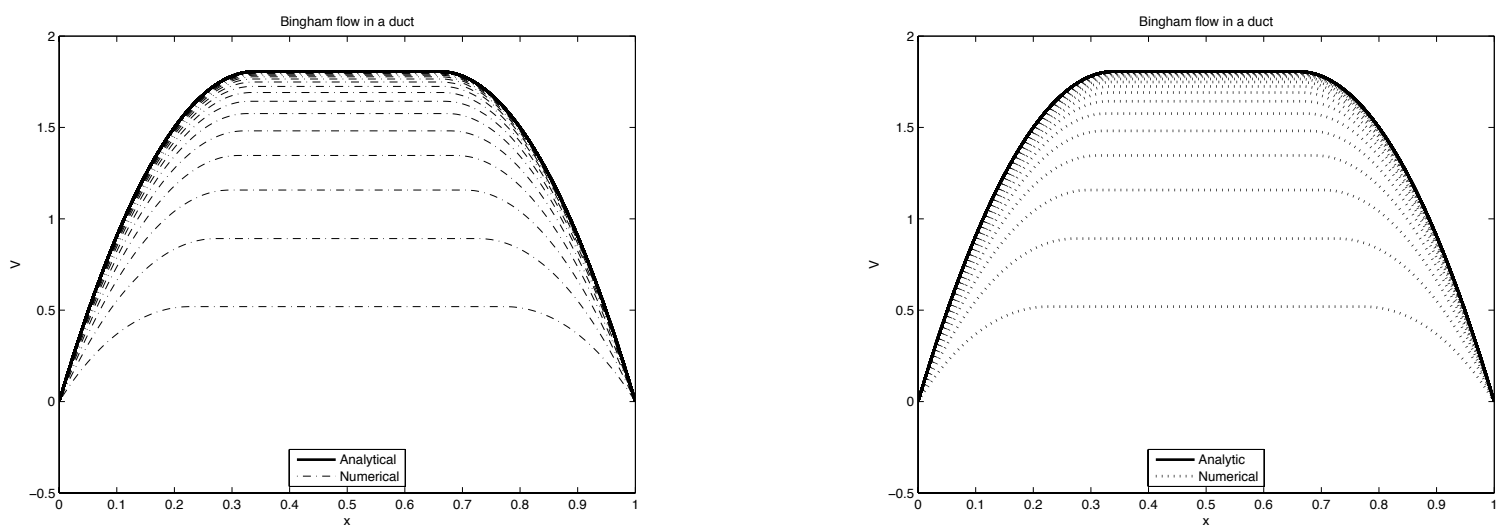

Figure F.16: Convergence of the computed velocity (dashed lines) to the analytical stationary solution (continuous line): (a) Augmented Lagrangian and (b) Bermúdez-Moreno methods. Note that there is a time $\Delta t=0.05$ between two successive curves. (See also Figure F.17.)

Indeed, we also determine the numerical order of convergence in space, through a mesh refinement study. This is done for the velocity and the associated multiplier for both duality methods. The results are given on Figures F.18 and F.19. We also give the associated figures in Tables F.5-F.6 and F.7-F.8, respectively. Note that the figures are the same for $V$ in Tables F.5 and F.7: this is normal since the methods are different but the solution that has to be found is unique and it was proven that they converge to this solution.

Essentially, the conclusions are that both the Augmented Lagrangian and the Bermúdez-Moreno methods are at least of order two (in $L^{2}$-norm) in space for the velocity of this non-zero stationary solution. Concerning the multiplier, which is only an auxiliary ingredient to compute the solution, we see more contrasted, but fairly good, results: on the one hand the Augmented Lagrangian method seems to be barely convergent but the error is very small $\left(10^{-9}-10^{-10}\right.$ in $L^{2}$-norm); on the other hand, the Bermúdez-Moreno method exhibits a second order convergence but the errors are 

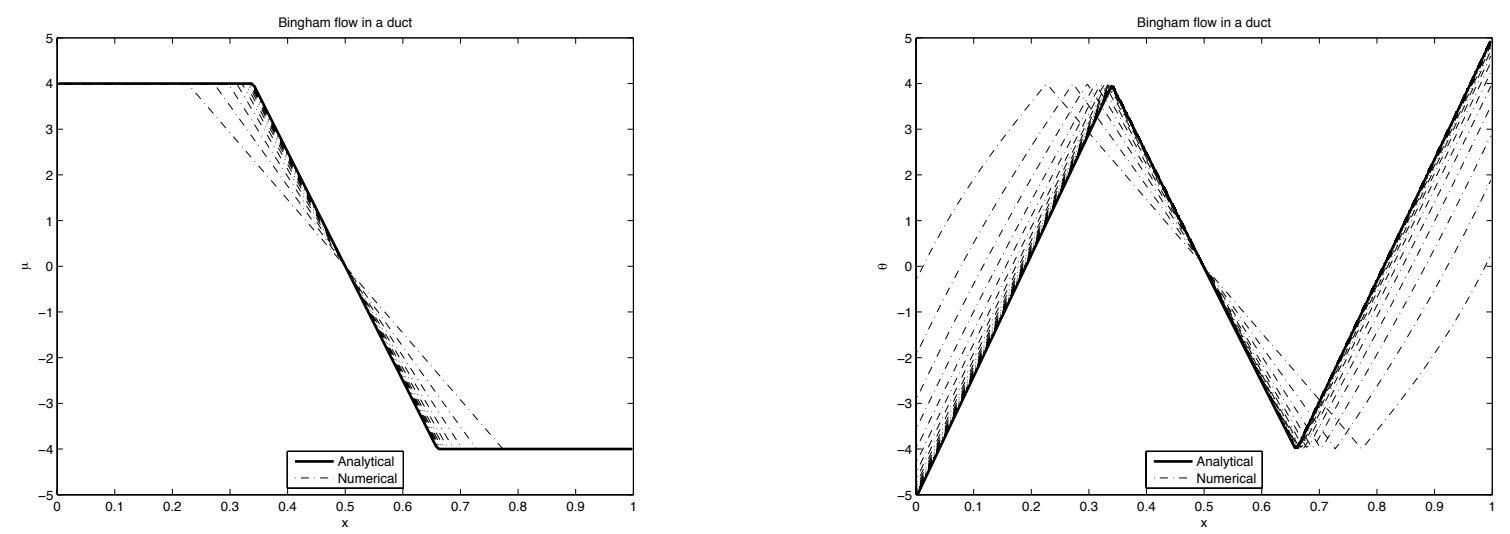

Figure F.17: Convergence of the computed multiplier (dashed lines) associated to the analytical stationary solution (continuous line): (a) Augmented Lagrangian and (b) Bermúdez-Moreno methods. Note that there is a time $\Delta t=0.05$ between two successive curves. (See also Figure F.16.)

much bigger than the Augmented Lagrangian method $\left(10^{-1}-10^{-7}\right.$ in $L^{2}$-norm).

Overall, this test is a first positive step validating the numerical ability of both methods to handle the variational inequality on $V$ with at least $L^{2}$-second order convergence in space.
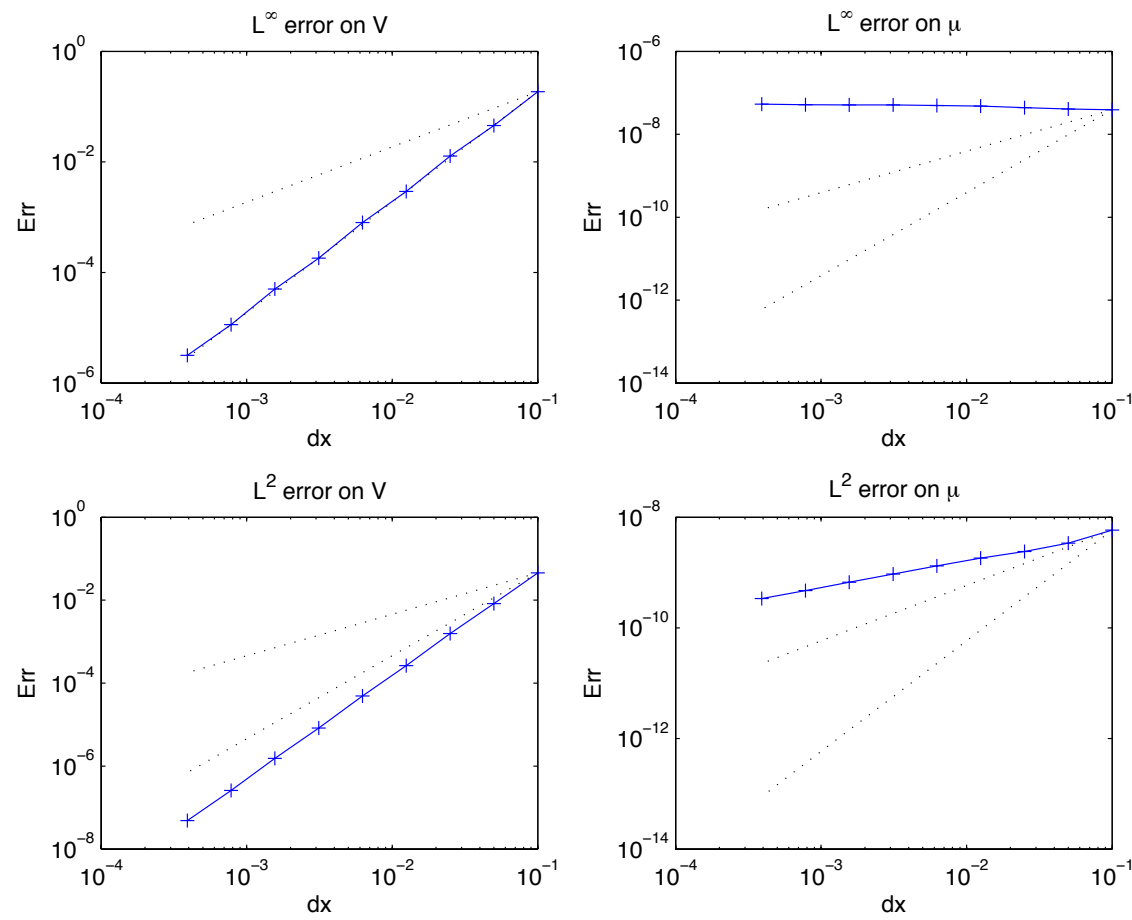

Figure F.18: Order of convergence under mesh refinement: Augmented Lagrangian method. Dotted lines show first and second order of convergence, blue line with + is the computed error. 
Table F.5: Test 1. Errors and order of convergence for Augmented Lagrangian - $V$.

\begin{tabular}{c|c|c|c|c}
\hline Cells & $L^{\infty}$ Error & Order & $L^{2}$ Error & Order \\
\hline 10 & $1.869 E^{-01}$ & - & $4.561 E^{-02}$ & - \\
20 & $4.558 E^{-02}$ & 2.036 & $8.284 E^{-03}$ & 2.460 \\
40 & $1.277 E^{-02}$ & 1.835 & $1.565 E^{-03}$ & 2.404 \\
80 & $2.915 E^{-03}$ & 2.131 & $2.645 E^{-04}$ & 2.566 \\
160 & $8.029 E^{-04}$ & 1.860 & $4.915 E^{-05}$ & 2.428 \\
320 & $1.825 E^{-04}$ & 2.137 & $8.282 E^{-06}$ & 2.569 \\
640 & $5.024 E^{-05}$ & 1.861 & $1.538 E^{-06}$ & 2.429 \\
1280 & $1.144 E^{-05}$ & 2.134 & $2.596 E^{-07}$ & 2.567 \\
2560 & $3.176 E^{-06}$ & 1.849 & $4.861 E^{-08}$ & 2.417 \\
\hline
\end{tabular}

Table F.6: Test 1. Errors and order of convergence for Augmented Lagrangian $-\mu$.

\begin{tabular}{c|cc|cc}
\hline Cells & $L^{\infty}$ Error & Order & $L^{2}$ Error & Order \\
\hline 10 & $3.903 E^{-08}$ & - & $5.817 E^{-09}$ & - \\
20 & $4.059 E^{-08}$ & -0.056 & $3.396 E^{-09}$ & 0.777 \\
40 & $4.410 E^{-08}$ & -0.120 & $2.398 E^{-09}$ & 0.502 \\
80 & $4.761 E^{-08}$ & -0.111 & $1.822 E^{-09}$ & 0.397 \\
160 & $4.959 E^{-08}$ & -0.059 & $1.316 E^{-09}$ & 0.469 \\
320 & $5.104 E^{-08}$ & -0.042 & $9.399 E^{-10}$ & 0.486 \\
640 & $5.129 E^{-08}$ & -0.007 & $6.645 E^{-10}$ & 0.500 \\
1280 & $5.160 E^{-08}$ & -0.009 & $4.704 E^{-10}$ & 0.498 \\
2560 & $5.350 E^{-08}$ & -0.052 & $3.378 E^{-10}$ & 0.478 \\
\hline
\end{tabular}

Table F.7: Test 1. Errors and order of convergence for Bermúdez-Moreno - $V$.

\begin{tabular}{c|cc|cc}
\hline Cells & $L^{\infty}$ Error & Order & $L^{2}$ Error & Order \\
\hline 10 & $1.869 E^{-01}$ & - & $4.561 E^{-02}$ & - \\
20 & $4.558 E^{-02}$ & 2.036 & $8.284 E^{-03}$ & 2.460 \\
40 & $1.277 E^{-02}$ & 1.835 & $1.565 E^{-03}$ & 2.404 \\
80 & $2.915 E^{-03}$ & 2.131 & $2.645 E^{-04}$ & 2.566 \\
160 & $8.029 E^{-04}$ & 1.860 & $4.915 E^{-05}$ & 2.428 \\
320 & $1.825 E^{-04}$ & 2.137 & $8.282 E^{-06}$ & 2.569 \\
640 & $5.024 E^{-05}$ & 1.861 & $1.538 E^{-06}$ & 2.429 \\
1280 & $1.144 E^{-05}$ & 2.134 & $2.596 E^{-07}$ & 2.567 \\
2560 & $3.176 E^{-06}$ & 1.849 & $4.860 E^{-08}$ & 2.417
\end{tabular}

We also study the computational cost of the two methods. To do so, for a given value of the parameter, we store the sum of the number of iterations done in the duality loop, for each iteration in time and up to the convergence to the stationary solution. This can be done, since the computational costs for one iteration in the duality loop are of the same order for both methods. (Recall also that for both methods, the number of iterations in time to reach the stationary solution is the same (up to a given precision, see (F.3))). The results are shown on Figure F.20 : with 

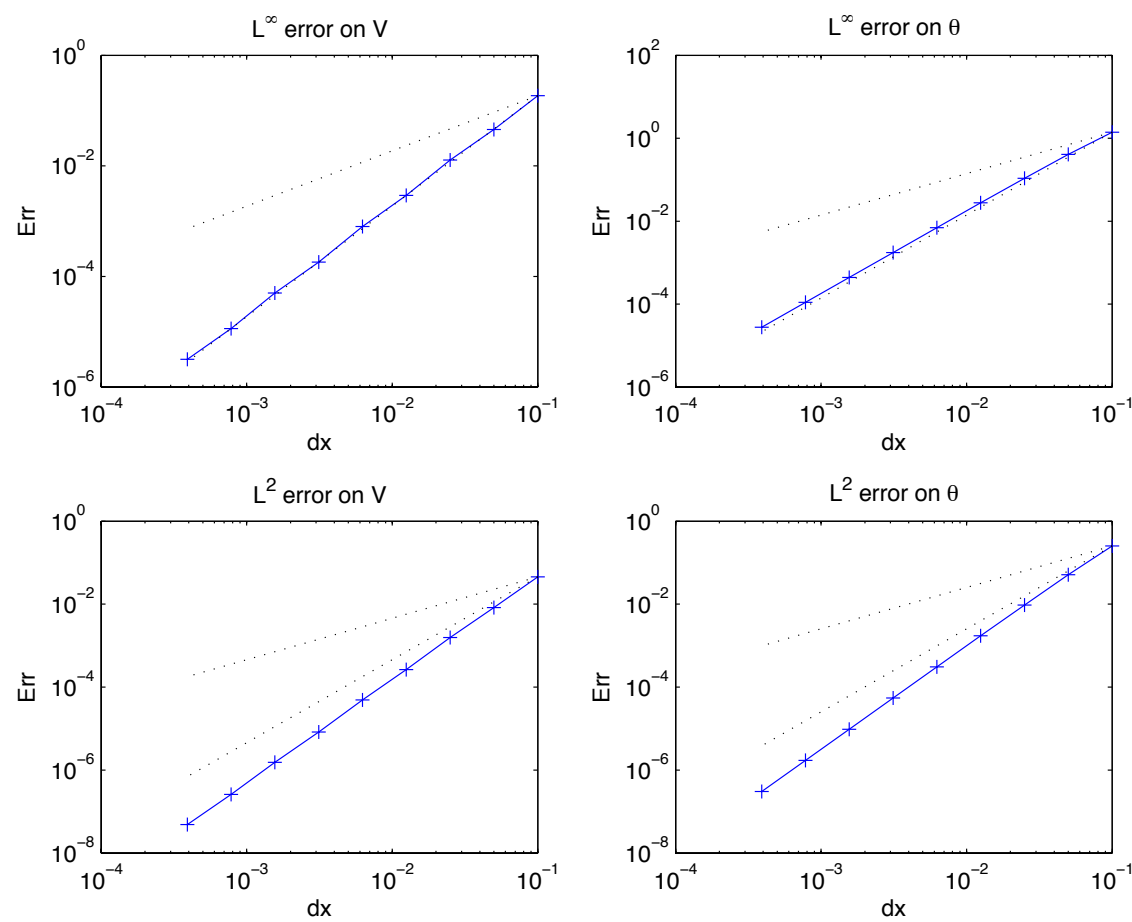

Figure F.19: Order of convergence under mesh refinement: Bermúdez-Moreno method. Dotted lines show first and second order of convergence, blue line with + is the computed error.

Table F.8: Test 1. Errors and order of convergence for Bermúdez-Moreno - $\theta$.

\begin{tabular}{c|cc|cc}
\hline Cells & $L^{\infty}$ Error & Order & $L^{2}$ Error & Order \\
\hline 10 & $1.400 E^{-00}$ & - & $2.530 E^{-01}$ & - \\
20 & $4.082 E^{-01}$ & 1.779 & $5.128 E^{-02}$ & 2.303 \\
40 & $1.081 E^{-01}$ & 1.917 & $9.529 E^{-03}$ & 2.428 \\
80 & $2.766 E^{-02}$ & 1.966 & $1.719 E^{-03}$ & 2.471 \\
160 & $6.988 E^{-03}$ & 1.985 & $3.065 E^{-04}$ & 2.487 \\
320 & $1.756 E^{-03}$ & 1.993 & $5.440 E^{-05}$ & 2.494 \\
640 & $4.402 E^{-04}$ & 1.996 & $9.640 E^{-06}$ & 2.497 \\
1280 & $1.104 E^{-04}$ & 1.996 & $1.709 E^{-06}$ & 2.496 \\
2560 & $2.778 E^{-05}$ & 1.990 & $3.042 E^{-07}$ & 2.490 \\
\hline
\end{tabular}

204 iterations, the Bermúdez-Moreno method is approximately $20 \%$ cheaper than the Augmented Lagrangian method (242 iterations).

In the case of the Bermúdez-Moreno method we can compare this numerical evaluation of $\omega$ with the theoretical estimation which gives $\omega_{\text {opt }}=1.44$; this is in very good agreement with the numerical investigation.

We can then study the well-balanced properties of the scheme in Section 5.1. 


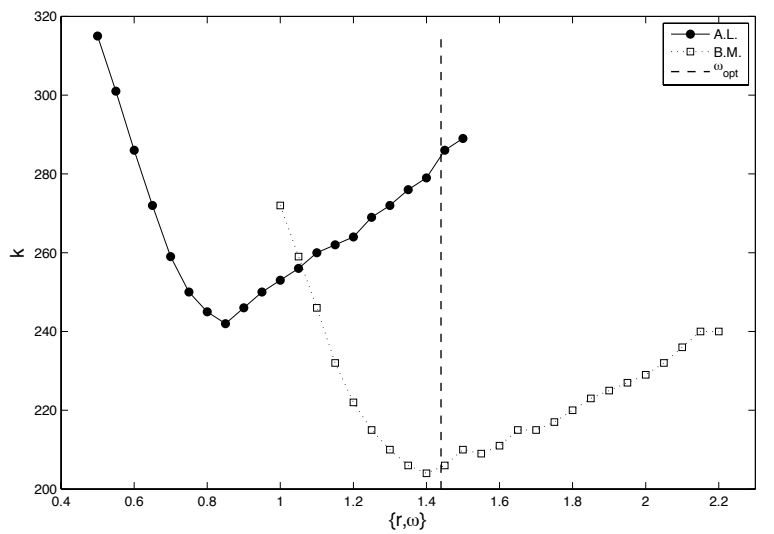

Figure F.20: Computational cost (number of iterations in the duality loops) with respect to the duality parameter: Augmented Lagrangian and Bermúdez-Moreno methods. Note that the value of the parameter realizing the minimum of the cost is (a) $r=0.85$ with $n b_{\text {iter }}=242$, (b) $\omega=1.4$ with $n b_{i t e r}=204$.

\section{References}

[1] C. Acary-Robert, E.D. Fernández-Nieto, G. Narbona-Reina, and P. Vigneaux. A well-balanced finite volume-Augmented Lagrangian method for an integrated Herschel-Bulkley model. Journal of Scientific Computing, 53:608-641, 2012 http://hal .archives-ouvertes.fr/ hal-00709491/en. 2, 10

[2] C. Ancey. Plasticity and geophysical flows: A review. Journal of Non-Newtonian Fluid Mechanics, 142:4 - 35, 2007.1

[3] N. J. Balmforth, A. S. Burbidge, R. V. Craster, J. Salzig, and A. Shen. Visco-plastic models of isothermal lava domes. J. Fluid Mech., 403:37-65, 2000. 4

[4] A. Bermúdez and C. Moreno. Duality methods for solving variational inequalities. Comput. Math. Appl., 7(1):43-58, 1981. 30, 32

[5] A. Bermúdez and M. E. Vázquez Cendón. Upwind methods for hyperbolic conservation laws with source terms. Comput. Fluids, 23(8):10491071, 1994. 2

[6] Daniele Boffi. Finite element approximation of eigenvalue problems. Acta Numer., 19:1-120, 2010. 33

[7] F. Bouchut. Nonlinear stability of finite volume methods for hyperbolic conservation laws and well-balanced schemes for sources. Birkhäuser, 2004. 2

[8] D. Bresch, E. D. Fernandez-Nieto, I. R. Ionescu, and P. Vigneaux. Augmented Lagrangian method and compressible visco-plastic flows: Applications to shallow dense avalanches. In G. P. Galdi et al., editor, New Directions in Mathematical Fluid Mechanics, Advances in Mathematical Fluid Mechanics, pages 57-89. Birkhauser Basel http://hal.archives-ouvertes.fr/hal-00327369/en, 2010. 2, 3

[9] H. Brézis. Opérateurs maximaux monotones et semi-groupes de contractions dans les espaces de Hilbert. North-Holland Publishing Co., 1973. 31

[10] M. J. Castro, J. M. Gonzáles-Vida, and C. Parés. Numerical treatment of wet/dry fronts in shallow flows with a modified Roe scheme. Math. Mod. Meth. App. Sci., 16(6):897-931, 2006. 2

[11] M.J. Castro, A.M. Ferreiro Ferreiro, J.A. García-Rodríguez, J.M. González-Vida, J. Macías, C. Parés, and M. Elena Vázquez-Cendón. The numerical treatment of wet/dry fronts in shallow flows: Application to one-layer and two-layer systems. Mathematical and Computer Modelling, 42(3-4):419-439, 2005. 2, 12

[12] T. Chacón, M. J. Castro, E. D. Fernández-Nieto, and C. Parés. On well-balanced finite volume methods for non-conservative nonhomogeneous hyperbolic systems. SIAM J. Sci. Comput., 29(3):1093-1126, 2007. 2, 10

[13] F. Delbos, J. Ch. Gilbert, R. Glowinski, and D. Sinoquet. Constrained optimization in seismic reflection tomography: a Gauss-Newton augmented Lagrangian approach. Geophysical Journal International, 164(3):670-684, 2006. 6

[14] J.D. Dent and T.E. Lang. A biviscous modified Bingham model of snow avalanche motion. Ann. Glaciol., 4:4246, 1983. 2

[15] G. Duvaut and J.-L. Lions. Inequalities in mechanics and physics. Springer-Verlag, 1976. 2, 4

[16] Ivar Ekeland and Roger Témam. Convex analysis and variational problems, volume 28 of Classics in Applied Mathematics. Society for Industrial and Applied Mathematics (SIAM), 1999. 31, 32

[17] Enrique D. Fernández-Nieto, José M. Gallardo, and Paul Vigneaux. Efficient numerical schemes for viscoplastic avalanches. Part 2: The 2D case. Accepted in Journal of Computational Physics, 2017 https://hal . archives-ouvertes . fr/hal-01593148/en - DOI. 5, 22

[18] Enrique D. Fernández-Nieto, Pascal Noble, and Jean-Paul Vila. Shallow water equations for Non-Newtonian fluids. Journal of NonNewtonian Fluid Mechanics, 165(1314):712 - 732, 2010. 4

[19] M. Fortin and R. Glowinski. Augmented Lagrangian methods: applications to the numerical solution of boundary-value problems. NorthHolland, 1983. 6, 17

[20] José M. Gallardo, Carlos Parés, and Manuel Castro. A generalized duality method for solving variational inequalities. Applications to some nonlinear Dirichlet problems. Numer. Math., 100(2):259-291, 2005. 2, 7, 30 
[21] J.-F. Gerbeau and B. Perthame. Derivation of viscous Saint-Venant system for laminar shallow water; numerical validation. Discrete Contin. Dyn. Syst., Ser. B, 1(1):89-102, 2001. 4

[22] R. Glowinski and P. Le Tallec. Augmented Lagrangian and operator-splitting methods in nonlinear mechanics, volume 9 of SIAM Studies in Applied Mathematics. Society for Industrial and Applied Mathematics (SIAM), 1989. 6, 29, 30

[23] R. Glowinski and A. Wachs. On the numerical simulation of viscoplastic fluid flow. In R. Glowinski and J. Xu, editors, Numerical Methods for Non-Newtonian Fluids, volume 16 of Handbook of Numerical Analysis, pages 483 - 717. Elsevier, 2011. 2,29

[24] J. M. Greenberg and A.-Y. Le Roux. A well-balanced scheme for the numerical processing of source terms in hyperbolic equations. SIAM J. Numer. Anal., 33(1):1-16, 1996. 2

[25] R. R. Huilgol and Z. You. Application of the augmented Lagrangian method to steady pipe flows of Bingham, Casson and Herschel-Bulkley fluids. Journal of Non-Newtonian Fluid Mechanics, 128(2-3):126 - 143, 2005. 2

[26] I. R. Ionescu. Augmented Lagrangian for shallow viscoplastic flow with topography. Journal of Computational Physics, $242: 544-560,2013$. 2

[27] Kazufumi Ito and Karl Kunisch. Lagrange Multiplier Approach to Variational Problems and Applications. Society for Industrial and Applied Mathematics, 2008. 6

[28] R. J. LeVeque. Balancing source terms and flux gradients in high-resolution Godunov methods: the quasi-steady wave-propagation algorithm. J. Comput. Phys., 146(1):346-365, 1998. 2

[29] Kirill D. Nikitin, Maxim A. Olshanskii, Kirill M. Terekhov, and Yuri V. Vassilevski. A numerical method for the simulation of free surface flows of viscoplastic fluid in 3D. Journal of Computational Mathematics, 29(6):605-622, 2011. 1

[30] T.C. Papanastasiou. Flows of materials with yield. J. Rheol., pages 385-404., 1987. 2

[31] Carlos Parés, Manuel Castro, and Jorge Macías. On the convergence of the Bermúdez-Moreno algorithm with constant parameters. Numer. Math., 92(1):113-128, 2002. 2, 3, 7, 8, 30, 32

[32] Carlos Parés, Jorge Macías, and Manuel Castro. Duality methods with an automatic choice of parameters. Application to shallow water equations in conservative form. Numer. Math., 89(1):161-189, 2001. 2, 7, 8, 30

[33] P. L. Roe. Upwind differencing schemes for hyperbolic conservation laws with source terms. In Nonlinear hyperbolic problems (St. Etienne, 1986). C. Carraso et al. (Eds), volume 1270 of Lecture Notes in Math., pages 41-51. Springer, Berlin, 1987. 2

[34] N. Roquet and P. Saramito. An adaptive finite element method for Bingham fluid flows around a cylinder. Computer Methods in Applied Mechanics and Engineering, 192(31-32):3317-3341, 2003. 2

[35] E. F. Toro. Shock-capturing methods for free-surface shallow flows. John Wiley \& Sons, 2001. 2

[36] G. Vinay, A. Wachs, and J.-F. Agassant. Numerical simulation of weakly compressible Bingham flows: The restart of pipeline flows of waxy crude oils. Journal of Non-Newtonian Fluid Mechanics, 136(2-3):93-105, 2006. 2

[37] D. Vola, F. Babik, and J.-C. Latché. On a numerical strategy to compute gravity currents of non-Newtonian fluids. J. Comput. Phys., 201(2):397-420, 2004. 2

[38] D. Vola, L. Boscardin, and J. C. Latché. Laminar unsteady flows of Bingham fluids: a numerical strategy and some benchmark results. J. Comput. Phys., 187(2):441-456, 2003. 2 